\title{
Modelos de equilibrio general y la incorporación de energías renovables
}

\author{
Disertación Pública. \\ Tesis que presenta \\ Elsa Patricia Omaña Pulido \\ Para obtener el grado de \\ Doctora en Ciencias y Tecnologías de la \\ INFORMACIÓN \\ Asesor: \\ Dr. Miguel Ángel Gutiérrez Andrade

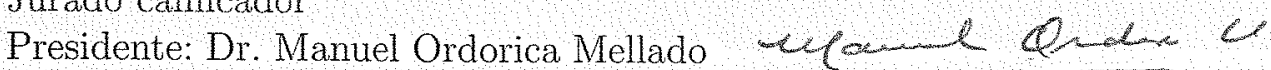

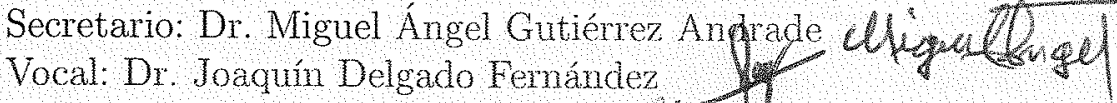 \\ Vocal: Dr. Pedro Lara Velázquez \\ Vocal: Dr. Francisco Venegas Martínez

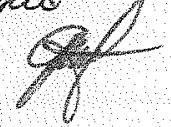

Jurado calificador

10 de noviembre de 2017 
Dedicado a mis hijas Fernanda y Amanda, porque me han enseñado otra parte de la vida 


\section{Resumen}

El objetivo de este trabajo es desarrollar un Modelo de Equilibrio General Computable (MEGC) para proponer políticas que permitan la integración óptima de las energías renovables, como un bien intermedio de los procesos de producción en el sistema económico mexicano. En algunas ocasiones estos modelos se pueden plantear como problemas de programación no lineal, pero de manera general se plantean como modelos complementarios mixtos

Se desarrollan un modelo estático y uno dinámico, para evaluar diferentes políticas fiscales a través de impuestos al uso de energías fósiles y subsidios al uso de las energías renovables. Para el caso dinámico resulta diferente y complicado el proceso de calibración de los parámetros, ya que los valores de éstos deben ajustarse para obtener el equilibrio de referencia.

El modelo estático muestra que la efectividad de una política fiscal que favorce la sustitución de las energías fósiles, por energías renovables, depende de la elasticidad de sustitución que existe en la economía, lo que además indujo a la incorporación de una elasticidad variable en el modelo dinámico. Los resultados obtenidos para el caso dinámico permiten determinar el porcentaje de variación del uso de las energías intermedias para medir la sustitución de las energías fósiles por renovables; así como analizar los impactos de esta sustitución en el ámbito macroeconómico. Se observa qué, a diferencia de la política impositiva al consumo de las energías de origen fósil, bajo la asignación de subsidios al uso de energías renovables, el PIB crece y el consumo agregado se mantiene constante comparado con el equilibrio de referencia dinámico.

Los modelos utilizan una matriz de contabilidad social, que representa la economía nacional y que para su empleo en este trabajo requirió previamente dos procesos. El primero consistió en agregar información de la matriz de insumo producto, en seis sectores representados por empresas; de los cuales dos producen bienes y servicios, dos energías fósiles, uno es generador de energía eléctrica y otro de energías renovables; el segundo radicó en la estimación e incorporación de la información agregada correspondiente a la demanda de las energías renovables como un bien intermedio. 


\section{Agradecimientos}

Este trabajo fue realizado parcialmente durante mi estancia en la División de Estudios de Posgrado de la Facultad de Economía de la UNAM, bajo la supervisión del Dr. Héctor M. Bravo Pérez, a quien agradezco su apoyo académico y el conseguir que me aceptaran en esta institución durante año y medio para realizar un periodo sabático. Agradezco también a los doctores Miguel Ángel Gutiérrez Andrade y Juan Carlos Castro Ramírez por el tiempo que dedicaron para enseñarme temas de Economía y el uso del modelador GAMS, y por sus valiosas sugerencias y comentarios para interpretar los resultados obtenidos, sin los cuales no hubiera podido desarrollar y alcanzar las metas planteadas.

Agradezco a los investigadores Dr. Joaquín Delgado Fernández, Dr. Francisco Venegas Martínez, Dr. Manuel Ordorica Mellado y Dr. Pedro Lara Velázquez; quienes se tomaron la molestia de leer este trabajo, preguntar sus dudas y hacer observaciones pertinentes alrededor de los temas que sustentan a la tesis.

Agradezco a mis hijas Fernanda y Amanda, y a Eduardo, por la paciencia y apoyo moral que me brindaron durante los años que estuve laborando en este proyecto académico. 


\section{Índice general}

Índice de figuras $\quad \mathrm{V}$

Índice de cuadros $\quad$ VI

1. Introducción $\quad 1$

2. Estado del arte $\quad 5$

2.1. El marco teórico de los MEGC . . . . . . . . . . . . . . . . . . . . . . . 6

2.2. Sustentabilidad energética . . . . . . . . . . . . . . 8

2.3. MEGC y sustentabilidad en México . . . . . . . . . . . . . . . . . 10

3. Modelo estático $\quad 13$

3.1. Descripción e hipótesis del modelo estático . . . . . . . . . . . . . . . . 13

3.2. Matriz de Contabilidad Social . . . . . . . . . . . . . . . . . 14

3.3. Modelos . . . . . . . . . . . . . . . . . . . 15

3.3.1. Productores . . . . . . . . . . . . . . . 16

3.3.2. Consumidor . . . . . . . . . . . . . . . 23

3.3.3. Otros agentes y bienes de consumo final . . . . . . . . . 25

3.4. Condiciones de equilibrio . . . . . . . . . . . . . . . . . . . . . . . 27

4. Políticas fiscales para el modelo Estático $\quad 29$

4.1. Calibración y Resultadoss . . . . . . . . . . . . . . . . . . 30

4.1.1. Calibración de los parámetros . . . . . . . . . . . . . . . . 32

4.2. Resultados para el modelo Estático . . . . . . . . . . . . . . . . . 34

4.2.1. Impuestos al uso de los bienes energéticos EPG y FPPC . . . . 34

4.2.2. Subsidio al uso de las energías renovables REN . . . . . . . . . . 42

5. Modelo dinámico $\quad 47$

5.1. Descripción e hipótesis del modelo dinámico . . . . . . . . . . . . . . . 47 
5.2. Modelos . . . . . . . . . . . . . . . . . . . . . . . . 48

5.2.1. Consumidor . . . . . . . . . . . . . . . . . . 49

5.2.2. Condiciones intertemporales e iniciales . . . . . . . . 50

5.3. Modelos Complementarios . . . . . . . . . . . . . . . . . 51

5.3.1. Problema complementario mixto . . . . . . . . . 51

5.3.2. Condiciones de frontera para un modelo con horizonte infinito . 52

5.4. Aproximación para un modelo Ramsey con horizonte infinito . . . . . . 55

6. Políticas fiscales para el modelo Dinámico 57

6.1. Calibración y Simulaciones . . . . . . . . . . . . . . . . . 58

6.2. Análisis y comparativo de la variación de las demandas intermedias . . 59

6.3. Análisis del impacto macroeconómico . . . . . . . . . . . . . . . 62

6.3.1. Impactos al gravar el uso de energías fósiles . . . . . . . . . 63

6.3.2. Impactos al asignar subsidios al uso de energías renovables . . . 67

$\begin{array}{ll}\text { 7. Conclusiones y Perspectivas } & 71\end{array}$

$\begin{array}{ll}\text { A. Anexo } & 77\end{array}$

A.1. Matriz de contabilidad social . . . . . . . . . . . . . . . 77

A.2. Desigualdades variacionales . . . . . . . . . . . . . . 80

A.3. Comparativo de la demanda de energías intermedias . . . . . . . . . . 82 


\section{Índice de figuras}

3.1. Anidación del proceso de producción _. . . . . . . . . . . . . . 17

4.1. Gráfica de las demandas de las energías bajo impuestos a la extracción de petróleo y gas, para $\sigma=1.8,10 \ldots \ldots \ldots \ldots \ldots$

4.2. Gráfica de las demandas de las energías bajo impuestos a la fabricación de productos derivados del petróleo y el carbón, para $\sigma=1.8,10 \ldots$.

4.3. Gráfica de las demandas de las energías bajo impuestos simultáneos a la EPG y la FPPC, para $\sigma=1.8,10 \ldots \ldots \ldots \ldots$

4.4. Gráfica de las demandas de las energías bajo asignación de subsidios al uso de las energías renovables, para $\sigma=0.5 \ldots \ldots \ldots$. . . . .

4.5. Comparativo de las demandas de energías bajo asignación de subsidios al uso de las energías renovables $\sigma=1.8 \ldots \ldots \ldots \ldots$

4.6. Comparativo de las demandas de energías bajo asignación de subsidios al uso de las energías renovables $\sigma=10 \ldots \ldots \ldots \ldots . \ldots 46$

6.1. Porcentaje de variación de la demanda intermedia del bien GEE . . . . 59

6.2. Porcentaje de variación de la demanda intermedia del bien EPG . . . . 60

6.3. Porcentaje de variación de la demanda intermedia del bien FPPC . . . 61

6.4. Porcentaje de variación de la demanda intermedia del bien REN . . . . 62

6.5. Porcentaje de variación del consumo agregado (Imp.) . . . . . . . . . 63

6.6. Porcentaje de variación de la inversión agregada (Imp.) . . . . . . . . . 64

6.7. Porcentaje de variación del PIB (Imp.) . . . . . . . . . . . . . . 65

6.8. Balanza comercial en porcentaje (Imp.) . . . . . . . . . . . . . 66

6.9. Porcentaje de variación del consumo agregado, (Sub.) . . . . . . . . 67

6.10. Porcentaje de variación de la inversión (Sub.) . . . . . . . . . . 68

6.11. Porcentaje de variación del PIB (Sub.) . . . . . . . . . . . . . . 69

6.12. Balanza comercial en porcentaje (Sub.) . . . . . . . . . . . . 70 


\section{Índice de cuadros}

4.1. Elasticidades de sustitución técnica . . . . . . . . . . . . . . . . . . 30

4.2. Coeficientes de proporcionalidad del factor compuesto . . . . . . . . . 33

4.3. Coeficientes de proporcionalidad y de escala en la producción de la energía agregada . . . . . . . . . . . . . . . . 33

4.4. Coeficientes de proporcionalidad en la función de utilidad de la familia 34

4.5. Impuesto a la extracción de petróleo y gas . . . . . . . . . . . . . 35

4.6. Impuesto a la Fabricación de Productos Derivados del Petróleo y del Carbón . . . . . . . . . . . . . . . . . . . . 36

4.7. Impuesto simultáneo a la producción de extracción de petróleo y gas y a la fabricación de productos derivados del petróleo y el carbón . . . . . . 37

4.8. Impuestos a la extracción de petróleo y gas para $\sigma=1.8,10 \ldots 38$

4.9. Impuestos a la fabricación de productos derivados del petróleo y el carbón,

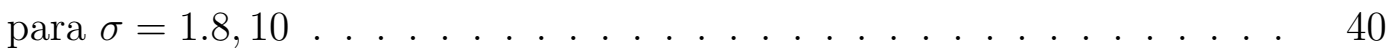

4.10. Impuestos simultáneos a los bienes energéticos EPG y FPPC, para $\sigma=$

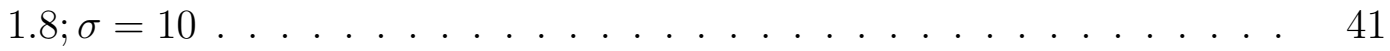

4.11. Subsidios al uso de las energías renovables . . . . . . . . . . . . . . 44

4.12. Subsidios al uso de las energías renovables $(\sigma=1.8 ; \sigma=10) \ldots \ldots$

A.1. Primera parte de la SAM, contiene a la submatriz de insumo-producto 77

A.2. Segunda parte de la SAM: ingresos de la familia por la renta de los factores y sus egresos . . . . . . . . . . . . . 78

A.3. Tercera parte de la SAM, egresos del gobierno, de las inversiones y del sector externo . . . . . . . . . . . . . . . . . . 79

A.4. Demanda de energías intermedias para elasticidad variable $\ldots . . . .82$

A.5. Indicadores macroeconómicos, Impuestos $(0-8$ años $) \ldots \ldots . . . . .83$

A.6. Indicadores macroeconómicos, Impuestos (9-16 años) . . . . . . . . . . 84

A.7. Indicadores macroeconómicos, Impuestos (17-25 años) . . . . . . . . . 84

A.8. Indicadores macroeconómicos, Subs. (0-8 años) . . . . . . . . . . . 85

A.9. Indicadores macroeconómicos, Subs. (9-16 años) … . . . . . . . 85 
A.10.Indicadores macroeconómicos, Subs. (17-25 años) … . . . . . . 86

A.11.Balanza comercial Imp. vs Subs. $(0-14$ años, $\%) \ldots \ldots \ldots$

A.12.Balanza comercial Imp. vs Subs. $(15-25$ años, $\%) \ldots \ldots \ldots$ 


\section{1 \\ Introducción}

En los últimos años, en el contexto de la economía ambiental se ha promovido la creación y desarrollo de nuevas líneas de investigación alrededor de temas relacionados con la sustentabilidad, como son la ecología, la explotación y manejo de los recursos no renovables, y en particular el uso y sustitución de las energías fósiles por energías renovables. Estos temas forman parte del trabajo de investigación de grupos interdisciplinarios que consideran como objetivo principal la explotación racional de los recursos, su impacto en el medio ambiente y sobre todo en el desarrollo económico mundial, por lo que casi todas las políticas derivadas consideran tratados internacionales como sistemas de regulación.

Una parte importante de la investigación desarrollada utiliza los llamados Modelos de Equilibrio General Computable (MEGC), tanto estáticos como dinámicos, que se sustentan en la teoría económica, Shoven [42]. En el caso de los modelos dinámicos se deben integrar además condiciones macroeconómicas que determinan el crecimiento económico, como son el crecimiento del capital, consumo, inversión y PIB; variables sobre las que se mide el impacto macroeconómico de las políticas económicas propuestas. En particular, los MEGC estáticos y dinámicos se pueden utilizar para estudiar la incorporación y efecto de impuestos y subsidios en los procesos productivos.

Cabe señalar que los MEGC se sitúan teóricamente tanto en la economía como en la matemática, ya sea utilizando modelos de la programación matemática o del cálculo variacional. En algunas ocasiones los MEGC contienen modelos de optimización en cuyo caso se utilizan modelos no lineales que se resuelven utilizando métodos de la programación no lineal. Cuando esto no sucede, se recurre a los llamados Modelos Complementarios Mixtos (MCM), (Mathiesen [22]; Lau, Pahlke y Rutherford[21]; Ferris et al. [12]), modelos que surgen en el contexto del cálculo variacional, desigualdades va- 
riacionales y problemas complementarios no lineales, ver Harker, P. T. y J.-S. Pang [18] (Anexo: Desigualdades variacionales), que por ser un sistema de ecuaciones no lineales también pueden ser resueltas con métodos y técnicas de programación matemática.

El objetivo de este trabajo es desarrollar un Modelo de Equilibrio General Computable (MEGC), para sustituir las energías fósiles por renovables; se analiza el impacto de la elasticidad de sustitución combinado con dos políticas fiscales. La primera política fiscal consiste en gravar el uso de energías no renovables como bienes intermedios en la producción del bien energía agregada; y la segunda asigna un subsidio a la utilización de energías renovables. En ambas políticas se supone que los impuestos a las energías fósiles se mantienen en el nivel definido por la economía de referencia. Los estudios que utilizan MEGC, requieren de la llamada matriz de contabilidad social para representar la economía en cuestión, en este caso la mexicana; la matriz correspondiente presentó limitaciones por lo que requirió de dos procesos previos a su uso. El primero consistió en agregar información de la matriz insumo producto en seis sectores, representados por empresas; el segundo radicó en estimar e incorporar en forma agregada la información contenida en documentos oficiales de la Secretaría de Energía (SENER) [41], correspondiente con la demanda de las energías renovables como un bien intermedio.

Los MEGC utilizados aquí, tanto estático como dinámico, implican una conducta optimizadora de los diferentes agentes económicos, y la solución numérica utiliza un sistema de ecuaciones obtenido por medio de los MCM, ya que las condiciones de primer orden de un problema de optimización coinciden con parte de ese sistema de ecuaciones. El modelo estático supone que tanto los procesos productivos de las empresas como la utilidad de los consumidores se representan por medio de una función Cobb-Douglas, y la producción del bien intermedio denominado energía agregada utiliza una función de elasticidad constante. El modelo dinámico, tipo Ramsey, asume las mismas condiciones de producción y consumo que el modelo estático, utiliza un horizonte de planeación infinito e incluye condiciones de tipo macroeconómico para medir el impacto de las políticas fiscales en el crecimiento económico. Finalmente, se destaca que los modelos desarrollados proporcionan a los investigadores de diferentes disciplinas, interesados en problemas relacionados con la reducción de emisiones de carbono, un marco teórico para desarrollar políticas de sustitución de energías fósiles por energías renovables; esto se alcanza al plantear un problema económico tomando en cuenta a los energéticos como un bien de producción intermedio o como un bien final, para satisfacer las necesidades de diferentes agentes económicos.

En México, durante el periodo del 2000 al 2010, el transporte fue el consumidor de energía con mayor crecimiento con un $43 \%$, seguido por la agricultura con el $24 \%$ según el reporte del 2013 de la Organización para la Cooperación y Desarrollo Económico (OCDE [24]). Actualmente existen algunos trabajos alrededor del tema del uso del carbono y la sustentabilidad, uno de los primeros fue desarrollado en 1994 por Romero [33], quien analiza una política medioambiental, basada en equilibrio general, que mide cuáles son los impactos del establecimiento de un impuesto ad valorem del $20 \%$ al uso del carbón; y concluye que disminuyen las emisiones totales de este contaminante en un 
$13 \%$, mientras que los impactos sobre el índice de precios y el PIB son mínimos. Por su parte, Boyd e Ibarrarán [9] utilizan un MEGC para evaluar el efecto de un impuesto al carbono en México, para mostrar que esta política disminuye la intensidad de las emisiones de carbono, sin pérdidas significativas en el bienestar social. También mediante un MEGC Bravo, Castro y Gutiérrez [10] evalúan el efecto en la distribución del ingreso de la economía mexicana, al aplicar un impuesto a la demanda de combustibles fósiles con el fin de disminuir la emisión de carbón a la atmósfera; en este último trabajo la aplicación del impuesto tiene el efecto deseado en la reducción de la demanda de combustibles fósiles, sin embargo no tiene un efecto significativo en la redistribución del ingreso. En México estos estudios, son los más relevantes en temas afines a la presente investigación y dos de ellos analizan los impactos en la redistribución de ingresos y en el bienestar social.

Este trabajo se desarrolla en seis capítulos adicionales a éste, el Capítulo 2 recapitula los resultados obtenidos en algunos de los trabajos más relevantes relacionados con MEGC, sustentabilidad y la planeación del uso de la energía. Los objetivos del Capítulo 3 son exponer el MEGC estático, que en este caso incluye los modelos de optimización de los agentes económicos productor y consumidor, y mostrar como las condiciones de primer orden y las condiciones de equilibrio definen un sistema de ecuaciones no lineales cuya solución determina la política de sustitución de energías fósiles, sistema que corresponde a las condiciones complementarias mixtas. Ligado al Capítulo 3, se desarrolla el Capítulo 4 en el que se presentan los resultados del modelo en cuadros y gráficas de las demandas de los bienes energéticos, que hacen posible realizar el análisis y conclusiones sobre la sustitución de dos energías de origen fósil por energías renovables, como bienes intermedios. Así la conclusión más relevante para el caso estático afirma que la efectividad de una política fiscal que favorece la sustitución de las energías fósiles, por el uso de energías renovables, depende de la elasticidad de sustitución que existe en la economía.

La descripción del modelo dinámico se realiza en el Capítulo 5, utiliza un horizonte de planeación infinito, que incluye condiciones de tipo macroeconómico para medir el impacto de las políticas fiscales en el crecimiento económico. En el Capítulo 6 se analizan los resultados obtenidos para el modelo dinámico. Este modelo requirió un proceso de calibración utilizando algunos parámetros definidos exógenamente por la economía mexicana que deben satisfacer una ecuación y que a la par permitan representar la economía de referencia expresada por la matriz de contabilidad social. Se supone que la elasticidad de sustitución es variable a lo largo del horizonte de planeación, tomando en cuenta la conclusión del modelo estático. La solución correspondiente aporta información sobre la producción de las energías intermedias bajo las dos políticas fiscales, que se interpretan en términos de la sustitución de las energías fósiles por renovables y además se analiza el impacto macroeconómico de esta sustitución para cada política. Los resultados muestran que la producción de los bienes energéticos de origen fósil decrece más rapidamente bajo impuestos que bajo subsidios.

La producción de la energía eléctrica es mayor bajo impuestos que bajo subsidios, 
pero el incremento de la producción de la energía renovable cuando hay subsidios es significativamente mayor. El análisis macroeconómico bajo impuestos muestra que las variables agregadas que representan el consumo, la inversión, y el PIB decrecen a mayor rapidez cuando el impuesto es mayor. Estas mismas variables crecen cuando se asignan subsidios al uso de las energías renovables. En términos de la balanza comercial resulta menos pernicioso optar por una asignación de subsidios al empleo de energías renovables que por un gravamen a la utilización de energías fósiles. Por lo tanto, a nivel macroeconómico resulta más eficiente asignar subsidios al uso de las energías renovables, que gravar al uso de las energías fósiles. Por último, el Capítulo 7 enuncia las conclusiones obtenidas en los capítulos 4 y 6, y algunas posibles líneas de investigación abiertas. 


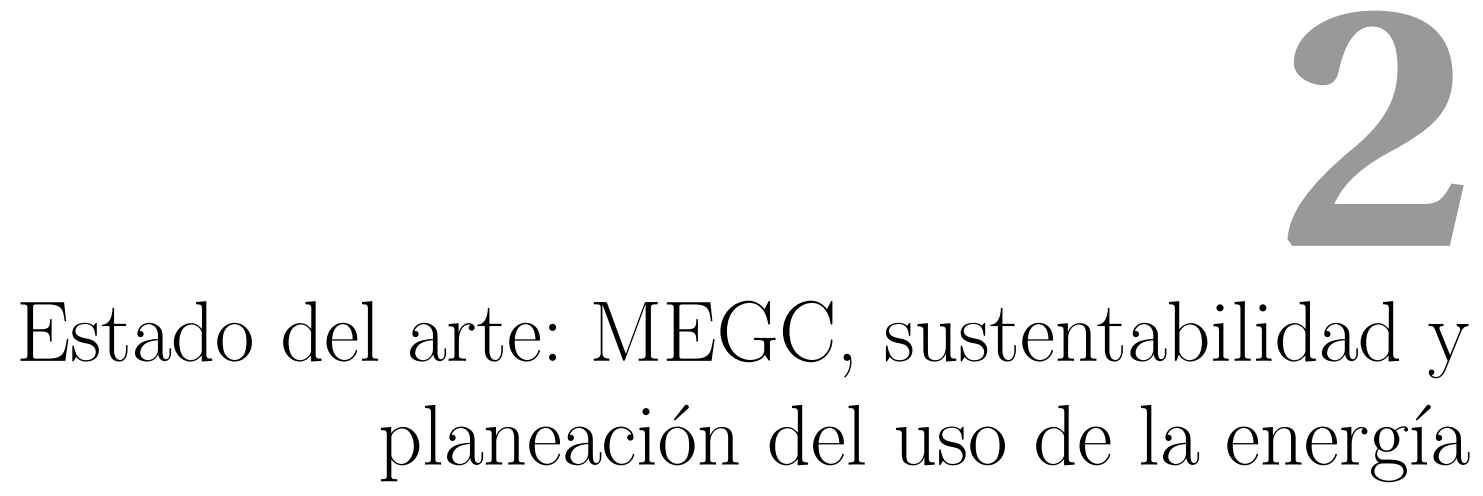

Los modelos de equilibrio general en economía, definidos por Arrow-Debrew [4], se utilizan para modelar el problema resuelto en este trabajo y están enmarcados por la teoría microeconómica, Shoven [42], y se denominan Modelos de Equilibrio General Computable (MEGC). En el proceso de planeación del uso de recursos no renovables se han desarrollado tanto MEGC estáticos como dinámicos, estos últimos son empleados para el proceso de planeación a mediano plazo, en el contexto económico los más recurridos son los modelos de Ramsey, Solow (Heer y Maussner [19], y Sala [38]) y Generaciones Traslapadas (Rausch y Rutherford [32]). Estos modelos por su naturaleza requieren además de la incorporación de condiciones macroeconómicas, se pueden desarrollar de manera determinista o estocástica; con horizonte finito o infinito, con variables discretas o continuas. Los modelos de Solow y Ramsey se distinguen porque se pueden utilizar para determinar el equilibrio general, en un contexto microeconómico, aunque los modelos de Ramsey tienen la ventaja de que incorporan la optimización intertemporal para analizar la función de utilidad del consumidor. Esta razón justifica que muchos de los modelos de crecimiento económico utilicen los modelos de Ramsey. Los modelos de generaciones traslapadas, se han utilizado para economías con agentes heterogéneos con una vida finita.

Para hacer una proyección a mediano plazo es más eficiente utilizar los modelos dinámicos, por lo que en este trabajo se usa concretamente un modelo tipo Ramsey. Existen diferentes aproximaciones de los modelos dinámicos, ver Heer y Maussner [19], Pearson et al. [29], Ginsburgh y Keyzer [14], Greenaway et al. [16]. Entre las herramientas computacionales que se han desarrollado para resolver tanto los problemas estáticos como dinámicos, Alex Meeraus inicia el desarrollo del modelador General Algebraic Mo- 
delin System (GAMS) y funda la Corporación de Desarrollo GAMS, este lenguaje de modelado algebraico fue evolucionando para resolver problemas de programación matemática y de condiciones complementarias para el caso estático. En particular, para los MEGC dinámicos Rutherford utiliza las condiciones complementarias para un modelo tipo Ramsey estable, en el sentido de que varias cantidades (capital, producción, consumos, etc.) crecen a razón constante, y posteriormente su alumno Paltsev en [26] expone un análisis y comparación de los modelos estáticos y dinámicos, y el uso del paquete GAMS para resolver ambos casos, para el caso dinámico se supone que los modelos son del tipo Ramsey y con un horizonte de planeación infinito (ver Rutherford T. [34], [35] y [36]).

En este capítulo se hace una reseña de los trabajos más relevantes de los MEGC, desde el sustento teórico que los fundamenta, y su uso en el ámbito de la sustentabilidad del medio ambiente y la energía.

\subsection{El marco teórico de los MEGC}

Los MEGC están fundamentados, por un lado en el marco de la micro y macroeconomía y por otro lado, en el contexto formal de la optimización y de la teoría del cálculo variacional. Esto se debe a que algunos de los MEGC incluyen modelos de optimización y otros no, se resalta que los modelos de optimización son un caso particular de los llamados Problemas de Desigualdades Variacionales (Sec. A.2). Se hace notar que en su inicio los problemas de desigualdades variacionales se situaron en el contexto del cálculo de variacional y en la teoría de control óptimo, en relación a condiciones de frontera representadas en un contexto de ecuaciones diferenciales parciales.

Si los MEGC incorporan modelos de optimización entonces las condiciones de primer orden, holgura complementaria y las condiciones de equilibrio económico cero beneficio, vaciado de mercados e ingresos balanceados definen un conjunto de igualdades y desigualdades no lineales que pueden ser resueltas por algoritmos y métodos propios de la investigación de operaciones, estudiados y desarrollados específicamente para problemas de optimización.

Cuando los MEGC no contienen modelos de optimización, debido a que no son integrables (ver el Anexo A), se plantean a través de otros casos particulares de los problemas de desigualdades variacionales, que pueden ser modelos complementarios no lineales, o lineales, o mixtos, o generalizados. Para estos modelos también se han desarrollado algoritmos y métodos, también situados en la investigación de operaciones. Algunos de ellos son métodos para ecuaciones no lineales, pero son restringidos a conjunto de ecuaciones que no incluyen desigualdades. Una metodología más versátil está basada en la teoría de punto fijo, que inicialmente fue desarrollada por Scarf en 1967 (ver [39]) y que posteriormente fue adaptada para determinar el equilibrio económico (ver [40]); para este mismo propósito Shoven utiliza técnicas de solución numérica como la del tanteo, o Gauss-Seidel, o Jacobi o Newton. Sin embargo, este tipo de metodologías 
pueden tener una eficiencia pobre para problemas de grandes dimensiones. Otra forma de abordar los problemas no integrables es a través de una sucesión de problemas integrables, para los cuales funcionan los métodos y algoritmos propios de la optimización, incluyendo métodos iterativos.

En 1985 Mathiesen [22] destaca que algunas de las características de los modelos no integrables son la existencia de desigualdades débiles y holgura complementaria, a este conjunto de desigualdades e igualdades los llama Problema Complementario Mixto denotado por MCP por su nombre en inglés, y desarrolla un método llamado Secuencia Lineal de Problemas Complementarios, que determina un equilibrio al resolver una sucesión de aproximaciones lineales para la expansión del polinomio de Taylor de primer orden para los términos no lineales del MCP. Lau et al. [21] usan la metodología de Mathiesen para resolver problemas de equilibrio general dinámico. Ferris et al. [12], hacen un análisis de modelos de programación matemática en los que se utilizan condiciones complementarias, así mismo indican que ciertos tipos de herramientas computacionales son recomendables, según sea el caso.

Rutherford aborda el sistema de desigualdades variacionales, en [35], al definir el caso particular del llamado Problema Complementario Mixto Generalizado (GMCP), que incluye cotas superiores e inferiores para ciertas variables. Los casos particulares de los MCP que aborda son: sistemas de ecuaciones lineales, sistemas de ecuaciones no lineales, problema complementario lineal, problema complementario no lineal, para el caso de problemas de optimización define el caso lineal, el no lineal. El método que utiliza Rutherford en su algoritmo permite resolver este tipo de problemas a gran escala.

En [35] se presenta cómo los problemas complementarios mixtos pueden ser resueltos con el simulador GAMS. Actualmente, se han desarrollado e incluído otros solvers para problemas complementarios mixtos de gran escala, MILES (Mixed Inequality and non Linear Equation Solver) y PATH. Respecto a la sintaxis hay dos interfaces, la relacionada con el GAMS que permite representar cualquier problema complementario y el MPSGE (Mathematical Programming System for General Equilibrium) que es utilizado para ecuaciones no lineales más específicas, que resultan de manera precisa en modelos de equilibrio económico.

Un aspecto importante, desde el punto de vista teórico, consiste en determinar bajo qué condiciones un problema de desigualdades variacionales puede contener un problema de programación matemática. Sin embargo, para los modelos económicos de equilibrio general hay condiciones específicas bajo las cuales no se pueden proponer conductas optimizadoras de los agentes económicos:

- Cuando hay más de un consumidor con diferentes preferencias y dotaciones.

- Existen restricciones institucionales sobre los precios, por ejemplo que deben permanecer en un rango.

- Existen impuestos y/o tarifas ad valorem. 
- Se exije que la acumulación del capital sea invariante a lo largo del horizonte de planeación, en el caso dinámico.

\subsection{MEGC y sustentabilidad energética}

Los modelos de equilibrio general computable (MEGC) han sido utilizados en diversos campos relacionados con la Economía; como la planeación del uso de energéticos, la sustitución de tecnologías, o la explotación de la tierra asignada al cultivo para obtener bio-energéticos. Gurgel et al. en [17], realizan un análisis de la integración de tierras vírgenes a campos de cultivo para incrementar la producción de biocombustibles, lo cual obviamente incrementa la deforestación, generando así únicamente un análisis del costo de conversión. Para apoyar la política de crecimiento de cultivos destinados a la generación de biocombustibles, Banse, Mejil, Tabeau y Woljer [5] hacen un análisis del impacto de esta política a nivel global, ya que los precios de estos cultivos impactan a nivel mundial. En particular, el análisis se realiza para medir el impacto que tiene esta política en el uso de la tierra en Europa.

Los MEGC también se utilizan para evaluar políticas ambientales como el impacto de impuestos y subsidios para disminuir la contaminación y mitigar los efectos del cambio climático. En este contexto, resalta el trabajo de Böhringer [7], quien ha trabajado en la utilización de los MEGC para la planeación del uso de los energéticos. En este documento se utiliza el análisis combinado "bottom-up y top-down" como una aproximación híbrida que permite estudiar la sustitución de patrones y la conversión de tecnologías más allá de una forma funcional, como las funciones del tipo CES (Constant Elasticity Sustitution). Posteriormente Böhringer y Rutherford [8] usan esta aproximación para modelos complementarios generalizados, con cotas superiores e inferiores sobre algunas variables de decisión, con el objetivo de analizar políticas energéticas y su impacto.

Algunas formas de abordar la problemática de sustitución de energéticos de origen fósil consiste en utilizar energías renovables y bio-energéticos; por ejemplo en [11] Dixon et al. utilizan modelos de equilibrio para investigar el impacto de sustituir, en motores, el uso de combustibles derivados del petróleo por combustibles basados en biomasa. Los autores hacen una proyección en la que hay una sustitución debido a que el precio de los combustibles a base de biomasa baja respecto a los precios de los combustibles fósiles, incluso a nivel mundial.

Kretschmer y Peterson [20], tienen un artículo fuente sobre las diferentes formas de abordar, en el marco de los modelos de equilibrio general, el uso de los biocombustibles en el desarrollo rural y su impacto para mitigar el calentamiento global y el impacto de su uso en la economía.

Govinda y Ram [15] utilizan un modelo estático de equilibrio general para analizar los impactos económicos y medioambientales del establecimiento de un impuesto al 
carbón en Tailandia; encontrándose que disminuyen el bienestar y la producción pero sorprendentemente casi no existen efectos sobre las emisiones de carbono.

Respecto a las emisiones del uso del carbón para generar energía y al carbón mismo, Rausch et al. [31] describen como el precio del carbón está determinado por la heterogeneidad tanto de las familias como de los factores de producción. En este artículo se analiza la distribución de las ganancias obtenidas dependiendo de la variación en los precios del carbón. Se hace notar que todo se desarrolla con un modelo estático de EGC.

Paul, Palmer y Woerman en [28] integran además de la reducción del bióxido de carbono $\left(\mathrm{CO}_{2}\right)$, la variación de precios y el abastecimiento de energía por región. En este trabajo el objetivo del gobierno es cambiar el uso de carbón para la generación de energía eléctrica, en particular Obama intentó incrementar hasta en un $80 \%$ la producción de electricidad a través de energías limpias, para el 2035. En este artículo se hace un análisis de diferentes políticas para alcanzar este objetivo, entre ellas se encuentran el cambio de tecnologías para abastecer la energía y el control en los precios de la electricidad. Al utilizar funciones de elasticidad constante los autores obtienen una reducción del $30 \%$ en las emisiones de $\mathrm{CO}_{2}$ para el periodo 2013-2035, y se reduce de manera dramática el uso de carbón en la generación de energía eléctrica. Simultáneamente, los precios se incrementan relativamente poco, sin embargo esto enmascara los impactos en las regiones. Actualmente, en EU la generación de energía eléctrica genera un $40 \%$ de las emisiones de $\mathrm{CO}_{2}$.

Los incentivos para generar políticas, de acuerdo a Palmer et al. (2010) [25] sobre el uso de energías alternativas en Estados Unidos (EU), son entre otros, la seguridad energética y el combate al cambio climático. El $40 \%$ de las emisiones de $\mathrm{CO}_{2}$ son generadas por el sector eléctrico y el $50 \%$ de la energía eléctrica es generada por carbón, sin embargo el cambio a energéticos no fósiles resulta caro, debido a que no se puede producir más energía en las horas pico, ni menos en las horas de poco consumo, esto representa poca flexibilidad de uso, y por lo tanto se debe sustituir al carbón tomando en cuenta esta limitante.

Popp et al. [30] revisan la literatura sobre el cambio tecnológico y el medio ambiente y encuentran que estos han recibido cada vez mayor atención. En su opinión, muchos de los problemas ambientales, como el cambio climático, son de largo plazo; lo que hace necesaria la comprensión de la evolución de la tecnología como parte importante de la proyección de los impactos a futuro. Aclaran que los problemas ambientales no pueden ser abordados, o sólo se podrán remitir a un gran costo, con el uso de las tecnologías existentes. Por ello es importante que se proporcionen incentivos para desarrollar nuevas tecnologías respetuosas del medio ambiente y que esto sea un objetivo de la política ambiental.

En el año 2012 Acemoglu et al. [2] desarrollan un modelo de crecimiento endógeno con cambio técnico el cual incluye restricciones ambientales, en éste se demuestra que una política dual en la cual se incluya impuestos al carbón y subsidios a la investigación 
es superior a una política basada únicamente en la modificación del precio del carbón; se sugiere también que dichas políticas de mitigación de daños al medioambiente necesitan aplicarse sólo temporalmente y sin sacrificar el crecimiento en el largo plazo.

Por su parte, en Acemoglu et al. [3], proponen un modelo en el cual se enfatiza la importancia que tiene, para lograr un crecimiento sustentable de la economía, la existencia de una alta elasticidad de sustitución entre los insumos energéticos renovables y los fósiles señalando que dicha elasticidad no ha sido estimada de manera sistemática. Un intento notable para remediar esta necesidad de información aparece en Saam et al. [37] donde se utiliza un panel de datos para una muestra de 29 países con el fin de estimar, por medio de funciones de producción CES anidadas, el parámetro de elasticidad y se concluye que, contrariamente a lo esperado por la literatura anterior sobre la sustituibilidad entre los combustibles fósiles, la evidencia empírica muestra una elasticidad de sustitución entre energías las renovables y fósiles mayor a uno. Así mismo mencionan que, para prevenir un incremento de la temperatura global mayor a dos grados Celsius, en un escenario factible, es recomendable que la elasticidad de sustitución tenga un valor de 10 .

Hourcade, et al. en [6] describen y concilian los enfoques de análisis top- down y bottom-up que son muy utilizados en una amplia bibliografía relacionada con planeación de energía (artículo que fue el resultado de un taller internacional llevado a cabo en Paris en abril del 2005). Ellos mencionan que los métodos híbridos han sido utilizados desde hace dos décadas y aun así no ha sido posible desarrolar un método donde se concreten todas las posibles estrategias desarrolladas hasta ahora.

En [13], Fullerton y Heutel estudian el impacto de impuestos por la generación de contaminantes con un enfoque de equilibrio general, en este caso se aborda la contaminación como un factor de producción con un cierto factor de sustitución con el trabajo y capital. En este artículo se determina los precios de los bienes de consumo y de los factores y se realiza un análisis del impacto a cambios en la intensidad del uso de los factores y los efectos de sustitución en las salidas. Se determinan las condiciones bajo las cuales el precio del trabajo incrementa el retorno del capital, se hace una asignación factible a los parámetros, y finalmente se determinan los rangos en los cuales varía la intensidad de uso de los factores para demostrar que tiene poco impacto comparado con los rangos de las elasticidades.

\subsection{MEGC y sustentabilidad en México}

Según el reporte del 2013 de la OCDE [24], en México durante el periodo del 2000 al 2010, el transporte fue el consumidor de energía con mayor crecimiento con un $43 \%$, seguido por la agricultura con el $24 \%$, lo que se refleja en un incremento de los gases de efecto invernadero y por lo tanto es deseable promover normas y políticas económicas que promuevan la sustentabilidad. En relación a la investigación hasta ahora desarrollada destaca Romero (1994) [33], quien analiza una política medioambiental, basada en 
equilibrio general, que mide cuáles son los impactos del establecimiento de un impuesto ad valorem del $20 \%$ al uso del carbón; concluyendo que disminuyen las emisiones totales de este contaminante en un $13 \%$, mientras que los impactos sobre el índice de precios y el PIB son mínimos. Posteriormente, Boyd e Ibarrarán [9] utilizan un modelo de equilibrio general computable para evaluar el efecto de un impuesto al carbono en México, mostrando que esta política disminuye la intensidad de las emisiones de carbono, sin pérdidas significativas en el bienestar social. También mediante un modelo de equilibrio general computable Bravo et al. [10] evalúan el efecto en la distribución del ingreso de la economía mexicana, al aplicar un impuesto a la demanda de combustibles fósiles con el fin de disminuir las emisiones de bióxido de carbón $\left(\mathrm{CO}_{2}\right)$ a la atmósfera; en este último trabajo la aplicación del impuesto tiene el efecto deseado en la reducción de la demanda de combustibles fósiles, sin embargo no tiene un efecto significativo en la redistribución del ingreso. 


\section{3 Modelo Estático}

Este capítulo tiene como objetivo describir el modelo de EGC estático que se utiliza para obtener los resultados numéricos que se analizan en el Capítulo 4; para ello aquí se definen los parámetros, las variables y las ecuaciones necesarias que describen la economía, definida por la llamada Matriz de contabilidad social o Social Accounting Matrix (MCS o SAM). Esta matriz representa todas las actividades económicas de producción y consumo.

\subsection{Descripción e hipótesis del modelo estático}

Se propone un modelo estático con seis empresas que representan a ciertos sectores económicos, dos factores de producción, trabajo y capital, y se supone que la economía es abierta. Los seis sectores se descomponen como dos sectores no energéticos el primario $(S P R)$ e industria y servicios (ISE). Los otros cuatro sectores son energéticos; dos de los cuales son productores de energías de origen fósil la extracción de petróleo y gas (EPG) y la fabricación de productos derivados del petróleo y del carbón (FPPC), otro es la generación, transmisión y suministro de energía eléctrica (GEE), y un sector productor de energía renovable (REN).

Las empresas producen bienes de consumo doméstico y de exportación, a excepción de REN que solamente produce un bien doméstico. La economía mexicana produce bienes domésticos para uso intermedio y para el consumo final de las familias y el gobierno. La SAM construída refleja que cada una de las empresas consume bienes intermedios de las cuatro industrias energéticas. Asimismo, la industria productora de energía eléctrica es la única que requiere energías renovables en su proceso productivo. Para simplificar 
la notación, los bienes intermedios que producen estos agentes se denotarán en forma genérica con las mismas siglas que las empresas.

En esta economía las familias son poseedoras de los factores de producción (trabajo y capital) y obtienen sus ingresos a partir de la renta de éstos, de las transferencias del gobierno y de las remesas del extranjero; este ingreso se destina al consumo de bienes o al ahorro, y al pago de impuestos. El gobierno actúa como un consumidor pasivo, en el sentido que su consumo no le proporciona utilidad alguna, obtiene sus ingresos por la recaudación de impuestos que posteriormente utiliza en el consumo de bienes finales, pago de factores de producción, transferencias a las familias, consumo de importaciones e inversión.

El objetivo general que se persigue es establecer políticas económicas que permitan sustituir el uso de las energías fósiles por el uso de las energías renovables, para reducir las emisiones de carbono. Entonces, al suponer que existen distintos grados de sustitución técnica en las funciones que representan la producción de la energía agregada, utilizando como bienes intermedios a las energías fósiles, eléctrica y renovables, se optó por usar funciones de elasticidad constante CES (Constant Elasticity Sustitution). La tecnología que se emplea para representar la producción total de cada empresa es una función tipo Cobb-Douglas, esta misma función se utilizó para representar la utilidad del consumo de las familias.

\subsection{Matriz de Contabilidad Social}

Cualquier sistema económico fundamenta sus estudios financieros, microeconómicos, macroeconómicos, etc. en la matriz de contabilidad social que lo representa. Esta matriz es un sistema de información estructurado, de acuerdo a lineamientos internacionales, en el que se reflejan las actividades económicas. Parte de la información refleja de manera precisa la interacción de los diferentes sectores productivos, en la llamada matriz de insumo producto, se estratifica a los diferentes consumidores de acuerdo a sus ingresos y preferencias de consumo, se contabilizan los ingresos del gobierno como recaudador de impuestos y sus egresos como provedoor de bienes y servicios, también se contabilizan los ingresos y egresos de un país ocasionados por tratados de comercio nacional e internacional. Resulta que esta matriz es un banco de datos fundamental para cualquier economía; la información que contiene se puede desagregar o agregar para realizar estudios económicos y financieros. En México, lamentablemente esta matriz no se ha mantenido actualizada debido a que algunos de los sectores económicos no renuevan la información que les corresponde.

El MEGC de este trabajo utiliza una matriz SAM (ver Anexo A) que se fundamenta en la construida por Aguayo, et al. [1], quienes emplean la Matriz de Insumo-Producto, (2004) del INEGI y la Encuesta nacional de ingresos y gastos de los hogares (ENIGH), 2004. En este artículo, en las páginas 226-227, se menciona la situación relativamente actual de la información de la SAM que representa la economía de México, así como las 
citas y referencias de quienes se han ocupado de realizar actualizaciones de esta matriz, de acuerdo a sus necesidades.

Cabe destacar que la matriz de contabilidad social SAM hecha por Aguayo et al. tuvo como objetivo la realización de un Análisis de la Generación y Redistribución del Ingreso en México, textualmente:

“...analizar la generación y redistribución de la renta en México y determinar el ingreso inducido y redistribuido de una inyección exógena unitaria como el caso de las remesas internacionales"

El proceso de construcción de la SAM empleada en esta investigación radica en modificar mediante dos procesos la SAM de Aguayo, et al. [1]. La primera parte consistió en agregar los datos, a seis sectores productivos; la segunda residió en estimar e incorporar en forma agregada la información contenida en documentos oficiales de la Secretaría de Energía (SENER, [41]), correspondiente con la producción de las energías renovables como un bien intermedio.

La matriz SAM de este trabajo considera como agentes económicos a:

1 Las seis empresas que representan a los seis sectores productivos, y cuya actividad se representa en la matriz de insumo-producto contenida en la SAM.

2 Un consumidor o familia representativa, dueña de los factores de producción, que utiliza parcialmente sus ingresos en el consumo de bienes producidos por los sectores SPR, ISE, GEE y FPPC.

3 El gobierno (GOB) recauda los impuestos que impone a cinco sectores y a la familia representativa por su consumo total. Los ingresos obtenidos los utiliza en el consumo de los bienes que producen las empresas, en pagos por trabajo y capital, en pago de importaciones, en transferencias a las familias a través de programas sociales y en ahorros.

En general, una matriz SAM se interpreta por renglón como los ingresos del sector o agente correspondiente a la fila; de manera análoga las columnas representan sus egresos. En el Anexo se muestra la matriz utilizada.

\subsection{Modelos}

En esta sección se describen los modelos del productor y del consumidor, que de forma integral representan parte de la dinámica del sistema económico. Cada productor tiene como objetivo minimizar los costos totales de su producción; los que se cuantifican como costos por la renta de los factores, costos de los bienes intermedios y costos de los bienes y servicios importados. La producción total de cada bien y las preferencias del 
consumidor se representan a través de una función Cobb-Douglas, lo que supone una cierta elasticidad de sustitución.

Los modelos que representan los objetivos de los productores y consumidores, en este caso se integran en el marco de la programación no-lineal y al utilizar las condiciones de primer orden, se generan dos conjuntos de ecuaciones que añadidos a las condiciones de equilibrio generan el sistema de ecuaciones denominado condiciones complementarias mixtas, a los que se refiere Mathiesen como el modelo complementario mixto MCP.

\subsubsection{Modelos para los productores}

La construcción del modelo del productor debe tomar en cuenta la información contenida en la matriz insumo producto de la SAM, para que una vez calibrado sea capaz de reproducir la economía contenida en esta matriz. Para alcanzar el objetivo central del trabajo, la sustitución de las energías fósiles por renovables, el modelo se desarrolla mediante un proceso anidado que se describe a continuación.

\section{Modelo anidado}

La anidación consiste en representar el proceso de producción para cada empresa como un árbol (Fig. 3.1), los vértices del nivel bajo se representan a los factores de producción y a los bienes energéticos. Los factores de producción se utilizan para elaborar el valor añadido por medio de una función Cobb-Douglas; los bienes energéticos sirven para producir la energía agregada a través de una función de elasticidad constante CES, que supone cierta elasticidad de sustitución. En ambos casos se persigue producir a costo mínimo. En el siguiente nivel, se encuentran como nodos intermedios los bienes primarios, los bienes de la industria y servicios, las importaciones, el valor añadido ligado a los factores, y la energía agregada conectada a los energéticos. Todos estos vértices, representan los bienes requeridos para llevar a cabo la producción total de cada industria, la que aparece como un nodo raíz. Se supone que la producción total de cada bien se rige utilizando una función Cobb-Douglas. Algunas referencias relacionadas con el uso de este proceso para el sector energético de USA se encuentran en Paltsev et al. [27] y en Mowers M. y Rausch [23]. 


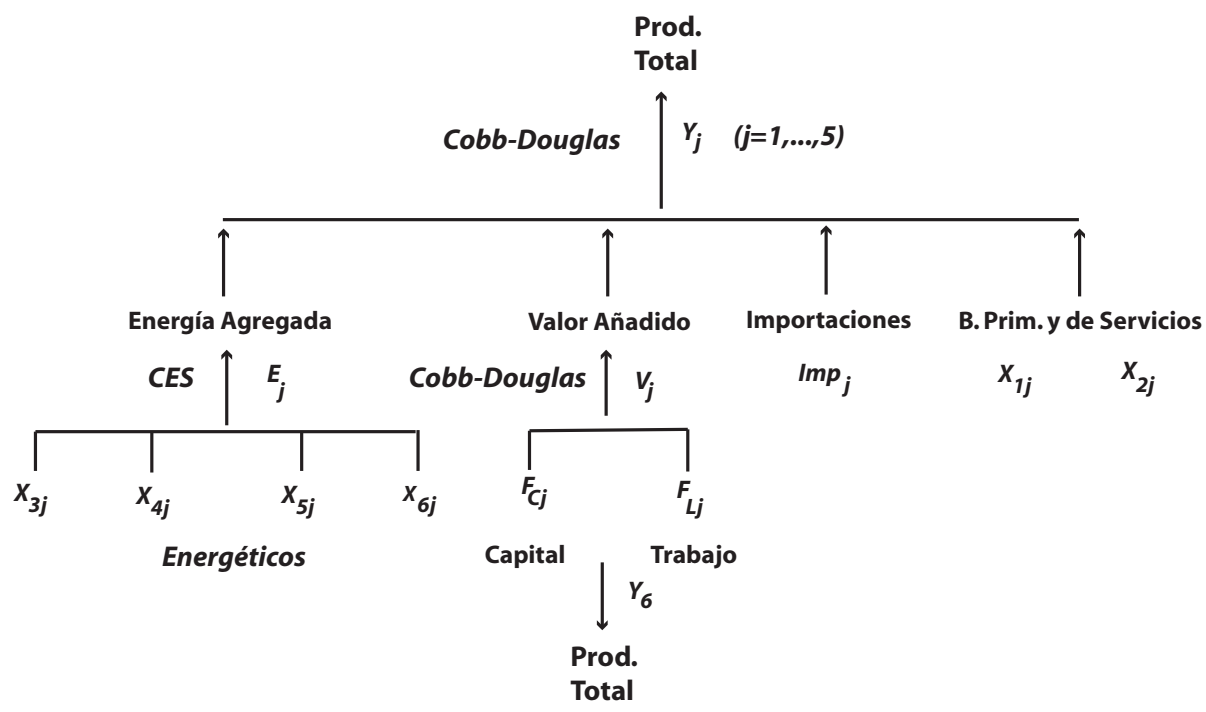

Figura 3.1: Anidación del proceso de producción

\section{Primera etapa: Producción del valor añadido}

La familia representativa ofrece el trabajo y capital para que las empresas utilicen estos factores, a través de una función Cobb-Douglas, en la producción del valor añadido; que debe alcanzar cierto nivel de producción. Entonces, el modelo es

$$
\min \sum_{k=L}^{C} w_{k} F_{k j}
$$

Sujeto a:

$$
V_{j}=B_{j} \Pi_{k=L}^{C} F_{k j}^{\beta_{k j}}
$$

donde $\sum_{k=L}^{C} \beta_{k j}=1$ para $\beta_{k j} \in[0,1]$.

La función objetivo representa el costo de los factores, los cuales combinados determinan el nivel de producción del valor añadido $V_{j}$ lo que queda establecido como la restricción correspondiente.

\section{Notación}

- $V_{j}$ denota el valor añadido, para $j=1, \ldots, 6$. 
- $F_{k j}$ es la demanda del factor de producción $k$ para producir el valor añadido $V_{j}$, para $k=L, C$ y para $j=1, \ldots, 6$.

- $B_{j}$ es el coeficiente de escala en la función de producción del valor añadido.

- $\beta_{k j}$ es el coeficiente de proporcionalidad del factor $F_{k j}$, para $k=L, C$, en la función de producción del valor añadido $V_{j}$, para $j=1, \ldots, 6$.

- $w_{k}$ es el precio del factor $F_{k j}$, para $k=L, C$.

SOLUCión:

El Lagrangiano para este modelo es:

$$
L\left(F_{k j}, V_{j}, \mu_{j}\right)=\sum_{k=L}^{C} w_{k} F_{k j}+\mu_{j}\left(V j-B_{j} \Pi_{k=L}^{C} F_{k j}^{\beta_{k j}}\right)
$$

Entonces, las condiciones de primer orden son:

$$
\begin{gathered}
\frac{\partial L\left(F_{k j}, V_{j}, \mu_{j}\right)}{\partial F_{k j}}=w_{k}-\mu_{j} \beta_{k j} \frac{B_{j} \Pi_{l=L}^{C} F_{l j}^{\beta_{l j}}}{F_{k j}}=0 \\
\frac{\partial L\left(F_{k j}, V_{j}, \mu_{j}\right)}{\partial \mu_{j}}=V j-B_{j} \Pi_{k=L}^{C} F_{k j}^{\beta_{k j}}=0
\end{gathered}
$$

La condición (3.2) exije la factibilidad del problema de optimización y la condición (3.1) afirma que $\mu_{j}$ es el precio sombra $v_{j}$ del valor añadido:

$$
\mu_{j}=v_{j}=\frac{w_{k} F_{k j}}{\beta_{k j} B_{j} \Pi_{l=L}^{C} F_{l j}^{\beta_{l j}}}=\frac{w_{k} F_{k j}}{\beta_{k j} V_{j}}
$$

Las variables $F_{L j}$ y $F_{C j}$ correspondientes a la demanda de los factores trabajo y capital se despejan de (3.3), cada uno se eleva a la potencia adecuada $\left(\beta_{L j}\right.$ y $\left.\beta_{C j}\right)$. Se multiplican entre ellos, y por $B_{j}$ generando la igualdad

$$
B_{j} F_{L j}^{\beta_{L j}} F_{C j}^{\beta_{C j}}=B_{j} \mu_{j}^{\beta_{L j}} \mu_{j}^{\beta_{C j}} V_{j}^{\beta_{L j}} V_{j}^{\beta_{C j}}\left[\frac{\beta_{C j}}{w_{C}}\right]^{\beta_{C j}}\left[\frac{\beta_{L j}}{w_{L}}\right]^{\beta_{L j}}
$$

se utiliza el hecho de que $\beta_{L j}+\beta_{C j}=1$, para obtener el valor añadido como

$$
V_{j}=B_{j} \mu_{j} V_{j}\left[\frac{\beta_{C j}}{w_{C}}\right]^{\beta_{C j}}\left[\frac{\beta_{L j}}{w_{L}}\right]^{\beta_{L j}}
$$


Por lo tanto, al despejar $\mu_{j}$ y usar que los coeficientes de proporcionalidad asociados a los factores satisfacen la igualdad $\beta_{L j}=1-\beta_{C j}$, se pueden deducir las siguientes igualdades

$$
\begin{gathered}
\mu_{j}=B_{j}^{-1}\left[\frac{w_{C}}{\beta_{C j}}\right]^{\beta_{C j}}\left[\frac{w_{L}}{\beta_{L j}}\right]^{1-\beta_{C j}}=B_{j}^{-1}\left[\frac{w_{C} \beta_{L j}}{w_{L} \beta_{C j}}\right]^{\beta_{C j}}\left[\frac{w_{L}}{\beta_{L j}}\right] \\
\mu_{j}=B_{j}^{-1}\left[\frac{w_{L} \beta_{C j}}{w_{C} \beta_{L j}}\right]^{\beta_{L j}}\left[\frac{w_{C}}{\beta_{C j}}\right]
\end{gathered}
$$

Por un lado, al sustituir las ecuaciones (3.5) y (3.6) en la ecuación (3.3), que define los factores $F_{L j}$ y $F_{C j}$ respectivamente, se obtiene el valor de estas variables de decisión,

$$
\begin{aligned}
& F_{C j}=V_{j} B_{j}^{-1}\left[\frac{w_{L} \beta_{C j}}{w_{C} \beta_{L j}}\right]^{\beta_{L j}} \\
& F_{L j}=V_{j} B_{j}^{-1}\left[\frac{w_{C} \beta_{L j}}{w_{L} \beta_{C j}}\right]^{\beta_{C j}}
\end{aligned}
$$

Por otro lado, la calibración de los coeficiente de participación $\beta_{k j}$ se obtiene a partir del cociente de la ecuaciones (3.8) y (3.7), al sustituir los valores de $F_{L j}$ y $F_{C j}$ que corresponden en la matriz SAM.

$$
\frac{F_{L j}}{F_{C j}}=\frac{w_{C} \beta_{L j}}{w_{L}\left(1-\beta_{L j}\right)}
$$

Finalmente, al reescribir la ecuación (3.2) como

$$
B_{j}=\frac{V_{j}}{\Pi_{k=L}^{C} F_{k j}^{\beta_{k j}}}
$$

el coeficiente de escala en la función de producción del j-ésimo valor añadido queda determinado por los coeficientes de participación, los factores de producción y el valor añadido definidos en la SAM. Por lo tanto las ecuaciones (3.9) y (3.10) permiten calibrar los parámetros asociados al valor añadido.

\section{Segunda etapa: Producción de la energía agregada}

Para precisar el modelo que sirve para determinar la producción de energía agregada $E_{j}$, para $j=1, \ldots, 5$ para cada empresa, se supone que $E_{j}$ está compuesta solamente por los bienes energéticos y dado que el objetivo es sustituir a las energías fósiles por renovables se supone que la demanda $X_{i j}$ de estos bienes intermedios se representa por medio de una función CES. El subíndice $i$ solamente debe considerar los bienes energéticos representados en los renglones $i=3, \ldots, 6$ de la SAM, y debido a que todas 
las empresas excepto la productora de energías renovables utilizan la energía agregada el subíndice $j$ satisface que $j=1, \ldots, 5$. Finalmente, la elasticidad de sustitución entre los bienes intermedios $X_{i j}$ está definida por el parámetro $\sigma_{j}$. Si el valor de este parámetro es menor que uno se dice que hay rigidez en la sustitución y si es mayor que uno se dice que es flexible, cabe aclarar que el parámetro de elasticidad no aparece explícitamente en el modelo. Entonces el modelo que permite determinar el nivel de producción de la energía agregada, y que define el nivel de sustitución de los bienes energéticos intermedios está definido como:

$$
\min \sum_{i=3}^{6} p_{i} X_{i j}
$$

Sujeto a:

$$
E_{j}=\psi_{j}\left(\sum_{i=3}^{6} \gamma_{i j} X_{i j}^{\rho_{j}}\right)^{\frac{1}{\rho_{j}}}
$$

en donde $\sum_{i=3}^{6} \gamma_{i j}=1$ para $\gamma_{i j} \in[0,1]$.

\section{Notación}

- $p_{i}$ denota el precio de la energía intermedia $X_{i j}$, para $i=3, \ldots, 6$, que requiere la producción de la energía agregada del sector $j=1, \ldots, 5$.

- $E_{j}$ es el nivel de la energía agregada que demanda el sector $j=1, \ldots, 5$.

- $\gamma_{i j}$ denota el coeficiente de proporcionalidad del bien intermedio $X_{i j}$ en la función de producción de la energía agregada $E_{j}$, para $i=3, \ldots, 6$.

- $\rho_{j}=\frac{\sigma_{j}-1}{\sigma_{j}}$ es el parámetro definido por la elasticidad de sustitución $\sigma_{j}$.

- $\psi_{j}$ es el coeficiente de escala en la función de producción de la energía agregada.

\section{SOLUCIÓN:}

El Lagrangiano de este modelo está definido como:

$$
\begin{gathered}
L\left(X_{3 j}, \ldots, X_{6 j}, E_{j}, \lambda_{j}\right)=\sum_{i=3}^{6} p_{i} X_{i j}-e_{j} E_{j} \\
-\lambda_{j}\left[E_{j}-\psi_{j}\left(\sum_{i=3}^{6} \gamma_{i j} X_{i j}^{\rho_{j}}\right)^{\frac{1}{\rho_{j}}}\right]
\end{gathered}
$$


Para construir el Lagrangiano se supone implícitamente que se debe satisfacer la condición de ingresos balanceados $\sum_{i=3}^{6} p_{i} X_{i j}=e_{j} E_{j}$, en donde $e_{j}$ denota el precio de la energía agregada $E_{j}$

Entonces, las condiciones de primer orden son:

$$
\begin{gathered}
\frac{\partial L}{\partial E_{j}}=-e_{j}+\lambda_{j}=0 \\
\frac{\partial L}{\partial \lambda_{j}}=E_{j}-\psi_{j}\left(\sum_{i=3}^{6} \gamma_{i j} X_{i j}^{\rho_{j}}\right)^{\frac{1}{\rho_{j}}}=0 \\
\frac{\partial L}{\partial X_{i j}}=p_{i}-\lambda_{j} \psi_{j} \gamma_{i j} X_{i j}^{\rho_{j}-1}\left(\sum_{i=3}^{6} \gamma_{i j} X_{i j}^{\rho_{j}}\right)^{\frac{1}{\rho_{j}}-1}=0
\end{gathered}
$$

La condición (3.11) reitera que el multiplicador de Lagrange $\lambda_{j}$ es el precio $e_{j}$ de la producción agregada $E_{j}$,

$$
\lambda_{j}=e_{j}
$$

La siguiente ecuación se infiere de la condición (3.12)

$$
\left(\frac{E_{j}}{\psi_{j}}\right)^{\rho_{j}}=\sum_{i=3}^{6} \gamma_{i j} X_{i j}^{\rho_{j}}
$$

Por lo tanto cualquiera de las dos condiciones que definen el valor de las variables $X_{i j}$, o bienes intermedios requeridos para la producir la energía agregada $E_{j}$, están dadas por

$$
\begin{aligned}
& X_{i j}=\left(\frac{p_{i}}{e_{j} \gamma_{i j} \psi_{j}^{\rho_{j}}}\right)^{\frac{1}{\rho_{j}-1}} E_{j} \\
& X_{i j}=\left(\frac{e_{j} \gamma_{i j} \psi_{j}^{\rho_{j}}}{p_{i}}\right)^{\frac{1}{1-\rho_{j}}} E_{j}
\end{aligned}
$$

las que se obtienen al sustituir las ecuaciones (3.14) y (3.15) en (3.13) y al despejarlas de esta misma ecuación.

El proceso que se requiere para determinar los parámetros $\gamma_{i j}$ para $i=3, \ldots, 6$ y $\psi_{j}$ consiste en plantear un sistema de ecuaciones en el que las variables son estos parámetros. En principio, se asume que los precios de las energías intermedias y de la energía agregada son unitarios. Al despejar el parámetro $\gamma_{i j}$ de la ecuación (3.17) 
se obtiene un sistema de cuatro ecuaciones, una para cada bien energético $E_{j}$ ( $j=$ $1, \ldots, 5)$.

$$
\gamma_{i j}=\frac{X_{i j}^{1-\rho_{j}} E_{j}^{\rho_{j}-1}}{\psi_{j}^{\rho_{j}}}
$$

para $i=3, \ldots, 6$

Debido a que los parámetros $\gamma_{i j}$ satisfacen

$$
\sum_{i=3}^{6} \gamma_{i j}=1
$$

de la ecuación (3.18), se deduce la igualdad

$$
\sum_{i=3}^{6} \gamma_{i j}=\frac{E_{j}^{\rho_{j}-1}}{\psi_{j}^{\rho_{j}}}\left[\sum_{i=3}^{6} X_{i j}^{1-\rho_{j}}\right]=1
$$

Por lo tanto, $\psi_{j}$ queda definada cuando se despeja de la ecuación previa

$$
\psi_{j}=E_{j}^{\frac{\rho_{j}-1}{\rho_{j}}}\left[\sum_{i=3}^{6} X_{i j}^{1-\rho_{j}}\right]^{\frac{1}{\rho_{j}}}
$$

El conjunto solución del sistema definido por las ecuaciones (3.18) y (3.19) determina el valor de los parámetros del modelo de producción tipo CES. Se hace notar que el parámetro $\rho_{j}$ se debe fijar de acuerdo al valor de la elasticidad de sustitución $\sigma_{j}$, de no ser así el sistema definido previamente tiene menos ecuaciones que variables.

\section{Tercera etapa: Producción óptima para cada sector}

El modelo a resolver para cada empresa tiene como objetivo minimizar los costos de producción sujeto a que la producción total alcanza un nivel de producción $Y_{j}$ definido por una función Cobb-Douglas, que se distingue porque el valor de la elasticidad es justamente uno.

$$
\min C_{j}=v_{j} V_{j}+\sum_{i=1}^{2} p_{i} X_{i j}+e_{j} E_{j}+p_{j} I m p_{j}
$$

Sujeto a:

$$
Y_{j}=D_{j}\left(\Pi_{k=1}^{2} X_{k j}^{\iota_{k j}}\right) V_{j}^{\iota_{v j}} E_{j}^{\iota_{e j}} \operatorname{Im} p_{j}^{\iota_{i j}}
$$


en donde $\sum_{k=1}^{2} \iota_{k j}+\iota_{v j}+\iota_{e j}+\iota_{i j}=1$ para los valores de $\iota_{k j}, \iota_{v j}, \iota_{e j}, \mathrm{y} \iota_{i j} \in[0,1]$.

y formalmente se resuelve para $j=1, \ldots, 5$.

\section{Notación}

- $Y_{j}$ es la cantidad total de la producción de la industria $j=1, \ldots, 5$.

- $C_{j}$ representa los costos totales de producción del bien final $Y_{j}$.

- $D_{j}$ denota el coeficiente de escala en la función de producción.

- $\iota_{k j}$ representa el coeficiente de proporcionalidad del bien intermedio $X_{k j}$ en la función de producción del bien final $Y_{j}$, para $k=1,2$.

- $\iota_{v j}, \iota_{i j}$ y $\iota_{e j}$ denotan respectivamente el coeficiente de proporcionalidad del valor añadido $V_{j}$, el coeficiente de proporcionalidad del bien intermedio de importación $I m p_{j}$ y el coeficiente de proporcionalidad de la energía agregada $E_{j}$, para producir una unidad de la producción total $Y_{j}$.

\subsubsection{Modelo para el consumidor}

La producción total $Y_{i}$ de cada sector se distribuye en producción para consumo externo o exportaciones $\operatorname{Exp}_{i}$ y para consumo doméstico. El consumo doméstico está compuesto por la demanda de la familia representativa $X_{i}$, la demanda del gobierno $X_{i g}$. La demanda de los bienes intermedios $X_{i j}$ y el bien de consumo final $I n v_{i}$ asociado a la inversión, para $i=1 \ldots, 5$ también forman parte del consumo doméstico. La producción de la empresa productora de energías renovables unicamente produce el bien intermedio $X_{63}$ para la empresa productora de energía eléctrica. Cabe señalar que el gobierno no se modela como un consumidor que obtenga utilidad por su consumo final, y debido a que ni la familia representativa ni el gobierno consumen el bien energético EPG las variables $X_{4}$ y $X_{4 g}$ son cero.

Se supone que las preferencias del consumidor se representan por medio de una función de utilidad Cobb-Douglas, estas preferencias no incluyen a las energías renovables ni a las relacionadas con la extracción de petróleo y gas. Entonces, el objetivo del consumidor es maximizar la función de utilidad, que depende de cuatro bienes de consumo final. Las restricciones en el modelo del consumidor generalmente están determinadas por su nivel de ingresos, el cual depende parcialmente de la renta de sus factores que en este caso son el trabajo y el capital. Además existen transferencias a las familias que son realizadas por el gobierno (por medio de programas sociales) y por el sector externo (como las remesas). Los egresos de la familia representativa están determinados por sus consumos y ahorro. Por lo tanto, el modelo está dado por:

$$
\max U\left(X_{1}, \ldots, X_{5}\right)=\prod_{i=1, i \neq 4}^{5} X_{i}^{\alpha_{i}}
$$


Sujeto a:

$$
(1+\tau) \sum_{i=1}^{5} p_{i} X_{i}+A_{p}=\sum_{k=L}^{C} w_{k} F_{k}+\left(T_{g}+T_{e}\right)
$$

donde $\sum_{i=1}^{5} \alpha_{i}=1$ y $\alpha_{i} \in[0,1]$.

Notación

- $p_{i}$ son los precios de los bienes de consumo final.

- $\alpha_{i}$ definen los coeficiente de proporcionalidad, del bien final $i=1,2,3,5$, en la función de utilidad de la familia.

- $A_{p}$ representa el ahorro de la familia.

- $\tau$ es la tasa que grava el consumo total de la familia.

- $T_{g}$ denota las transferencias del gobierno a la familia.

- $T_{e}$ representa las transferencias del sector externo a la familia.

\section{SOLUCIÓN}

El Lagrangiano para este modelo es:

$$
\begin{gathered}
L\left(X_{1}, \ldots, X_{5}, \zeta\right)=\prod_{i=1}^{6} X_{i}^{\alpha_{i}} \\
-\zeta\left[(1+\tau) \sum_{i=1}^{5} p_{i} X_{i}+A_{p}-\sum_{k=L}^{C} w_{k} F_{k}-\left(T_{g}+T_{e}\right)\right]
\end{gathered}
$$

Entonces, las condiciones de primer orden son:

$$
\frac{\partial L}{\partial \zeta}=(1+\tau) \sum_{i=1}^{5} p_{i} X_{i}+A_{p}-\left(\sum_{k=L}^{C} w_{k} F_{k}+T_{g}+T_{e}\right)=0
$$

En donde $\zeta$ es el precio sombra del consumo de la familia representativa.

$$
\frac{\partial L}{\partial X_{i}}=\alpha_{i} \frac{\Pi_{j=1}^{5} X_{j}^{\alpha_{j}}}{X_{i}}-\zeta(1+\tau) p_{i}=0
$$

La ecuación (3.20) representa la factibilidad del problema y es de interés reescribirla como:

$$
\sum_{i=1}^{5} p_{i} X_{i}=\left[\frac{\sum_{k=L}^{C} w_{k} F_{k}-A_{p}+T_{g}+T_{e}}{(1+\tau)}\right]
$$


La condición (3.21) se utilizará en la siguiente forma:

$$
\alpha_{i} \frac{\prod_{j=1}^{5} X_{j}^{\alpha_{j}}}{(1+\tau) \zeta}=p_{i} X_{i}
$$

Así al realizar la suma para $i=1, \ldots, 5$ en esta ecuación se obtienen las igualdades

$$
\sum_{i=1}^{5} p_{i} X_{i}=\left[\frac{\Pi_{j=1}^{5} X_{j}^{\alpha_{j}}}{(1+\tau) \zeta}\right] \sum_{i=1}^{5} \alpha_{i}=\frac{\Pi_{j=1}^{5} X_{j}^{\alpha_{j}}}{(1+\tau) \zeta}
$$

A partir de la ecuación (3.22) y de la ecuación (3.24) se determina la igualdad

$$
\frac{\Pi_{j=1}^{5} X_{j}^{\alpha_{j}}}{(1+\tau) \zeta}=\frac{\sum_{k=L}^{C} w_{k} F_{k}-A_{p}+T_{g}+T_{e}}{(1+\tau)}
$$

y al utilizar la ecuación (3.23) la igualdad anterior se puede reescribir como

$$
\frac{p_{i} X_{i}}{\alpha_{i}}=\frac{\sum_{k=L}^{C} w_{k} F_{k}-A_{p}+T_{g}+T_{e}}{(1+\tau)}
$$

Por lo tanto, la variable de decisión $X_{i}$ que determina el nivel de demanda de los bienes de consumo de las familias es

$$
X_{i}=\frac{\alpha_{i}}{p_{i}}\left[\frac{\sum_{k=L}^{C} w_{k} F_{k}-A_{p}+T_{g}+T_{e}}{(1+\tau)}\right]
$$

para $i=1, \ldots, 5, i \neq 4$.

Análogamente, el valor del parámetro que determina el coeficiente de proporcionalidad es

$$
\alpha_{i}=(1+\tau)\left[\frac{p_{i} X_{i}}{\sum_{k=L}^{C} w_{k} F_{k}-A_{p}+T_{g}+T_{e}}\right]
$$

para $i=1, \ldots, 5$

\subsubsection{Otros agentes y bienes de consumo final}

\section{Gobierno}

El consumo del gobierno no se representa a través de un modelo, sin embargo satisface condiciones de déficit cero. En lo que respecta a sus ingresos, los obtiene a partir de los impuestos que recauda al gravar la producción total de las empresas y al consumo de las familias. Generalmente los impuestos se dividen en impuestos directos que gravan la 
renta del consumidor (ISR) e indirectos que gravan al consumo (IVA), en este trabajo no se consideró necesario separarlos por lo que los impuestos para cada industria están agregados en un único valor. En la siguiente igualdad se incluye el parámetro $\tau_{i j}$ que representa el impuesto al uso de las energías fósiles o subsidio al uso de las renovables, este parámetro no pertenece a la economía de referencia.

Entonces, la recaudación total es la suma de los ingresos parciales y debe ser igual a sus egresos:

$$
\begin{gathered}
\sum_{j=1}^{5} \tau_{j} p_{j} Y_{j}+\sum_{i=1}^{6} \sum_{j=1}^{5} \tau_{i j} p_{i} X_{i j}+\tau \sum_{i=1}^{4} p_{i} X_{i}= \\
\sum_{i=1}^{5} p_{i} X_{i g}+\sum_{k=L}^{C} w_{k} F_{k g}+T_{g}+A_{g}+I_{m} p_{g}
\end{gathered}
$$

- $\tau_{j}$ es la tasa que grava la producción total de los sectores.

- $\tau_{i j}$ es la tasa que grava o subsidia el bien $i$ utilizado para producir el bien $j$.

- $\tau$ denota la tasa del impuesto al consumo total de las familias.

- $A_{g}$ es el ahorro del gobierno.

- $I m p_{g}$ son las importaciones del gobierno.

- $F_{k g}$ son los factores que utiliza el gobierno.

\section{Inversiones}

La inversión de esta economía, como bien de consumo final de las empresas, está compuesta por el ahorro $A_{p}$ de la familia, el ahorro público $A_{g}$ que proviene del gobierno, y los ahorros del sector externo $A_{s e}$. Este ahorro se distribuye como ingresos para los productores y el sector externo. Este proceso se representa mediante la ecuación

$$
A_{p}+A_{g}+A_{s e}=\sum_{j=1}^{5} \operatorname{Inv}_{j}+T A_{e}
$$

\section{Notación}

- $I n v_{j}$ son ingresos que reciben los sectores productivos de las cuentas de inversión.

- $T A_{e}$ denota las transferencias del ahorro al sector externo. 


\section{Sector Externo}

Este sector produce bienes de consumo para los productores de bienes intermedios, las familias y el gobierno. También recibe ingresos del pago neto de los factores del exterior $T_{c}$ y de las transferencias del ahorro al sector externo. Sus egresos se deben a los consumos de las exportaciones $E x p_{j}$ de los sectores productivos, las transferencias a la familia y los ahorros que realiza.

$$
\sum_{j=1}^{5} I m p_{j}+I m p_{g}+T A_{e}+T_{c}=\sum_{j=1}^{5} \operatorname{Exp}_{j}+T_{e}+A_{s e}
$$

\subsection{Condiciones de equilibrio}

El equilibrio se define como un conjunto de vectores de precios; los precios de bienes de consumo final $\mathbf{p}^{*}=\left(p_{1}, \ldots, p_{6}\right)$ que también corresponden a los precios de bienes intermedios, y los precios de los factores de producción $\mathbf{w} *=\left(w_{L}, w_{C}\right)$. También incluye a los vectores de la producción total $\mathbf{Y}^{*}=\left(Y_{1}, \ldots, Y_{6}\right)$, de los bienes finales $\mathbf{X}=$ $\left(X_{1}, \ldots, X_{5}\right)$, de los bienes intermedios $\mathbf{X}_{\mathbf{i}}^{*}=\left(X_{i j}\right)$ para $i=1, \ldots, 5$ y para $j=1, \ldots, 6$ y el correspondiente a los factores de producción $\mathbf{F}^{*}=\left(F_{L j}, F_{C j}\right)$; vectores que deben satisfacer una asignación óptima. Todos estos vectores satisfacen las condiciones de equilibrio:

Condiciones de vaciado de mercados

- Mercado de bienes. Para cada empresa su producción total satisface

$$
Y_{i}=\sum_{j=1}^{5} X_{i j}+X_{i}+X_{i g}+\frac{\operatorname{Inv}_{i}}{p_{i}}+\frac{E x p_{i}}{p_{i}}
$$

para $i=1, \ldots, 5$

- Mercado de factores. Para cada factor $k$, el total de este factor $F_{k}$ cumple

$$
F_{k}=\sum_{j=1}^{6} F_{k j}+F_{k g}
$$

para $k=L, C$ 
Condiciones de cero beneficio

$$
p_{j} Y_{j}=v_{j} V_{j}+\sum_{i=1}^{2} p_{i} X_{i j}+e_{j} E_{j}+p_{j} I m p_{j}
$$

para todos los sectores $(j=1, \ldots, 6)$.

\section{Asignación óptima}

- La asignación de la demanda para el consumo final es óptima,

$$
X_{1}, \ldots, X_{5} \in \arg \max \left\{U\left(X_{1}, \ldots, X_{5}\right):(1+\tau) \sum_{i=1}^{5} p_{i} X_{i}+A_{p}=\sum_{k=L}^{C} w_{k} F_{k}+T_{g}+T_{e}\right\}
$$

- La asignación de factores $F^{*}=\left(F_{L j}, F_{C j}\right)$ y de bienes intermedios $\left(X_{i 1}, \ldots, X_{i 5}, X_{63}\right)$ para $i=1, \ldots, 5$ es óptima,

$$
V_{j}, X_{i j}, E_{j}, I m p_{j} \in \arg \min \left\{C_{j}: Y_{j}=D_{j}\left(\Pi_{k=1}^{2} X_{k j}^{\iota_{k j}}\right) V_{j}^{\iota_{v j}} E_{j}^{\iota_{e j}} I m p_{j}^{\iota_{i j}}\right\}
$$

- El gobierno incurre en déficit cero

$$
\begin{gathered}
\sum_{j=1}^{5} \tau_{j} p_{j} Y_{j}+\sum_{i=1}^{6} \sum_{j=1}^{5} \tau_{i j} p_{i} X_{i j}+\tau \sum_{i=1}^{4} p_{i} X_{i}= \\
\sum_{i=1}^{5} p_{i} X_{i g}+\sum_{k=L}^{C} w_{k} F_{k g}+T_{g}+A_{g}+\operatorname{Im} p_{g}
\end{gathered}
$$

- Para el sector externo los ingresos son iguales a los egresos

$$
\sum_{j=1}^{5} I m p_{j}+I m p_{g}+T A_{e}+T_{c}=\sum_{j=1}^{5} E x p_{j}+T_{e}+A_{s e}
$$

Como se mencionó al inicio de este capítulo, las ecuaciones que determinan las variables de decisión, obtenidas por las condiciones de primer orden, de los modelos del consumidor y del productor, las condiciones de equilibrio y las condiciones que se satisfacen para los otros agentes del sistema económico forman un sistema de ecuaciones cuya solución determina la política óptima de producción y consumo de la economía. 


\section{4 \\ Resultados para el modelo Estático}

En este capítulo se analizan y evalúan los resultados al utilizar dos políticas fiscales diferentes, con el propósito de sustituir energías fósiles por energías renovables en la producción del bien energía agregada $E_{j}$, para cada industria $j$, la cual se modela por medio de una función CES. Cada una de estas políticas debe suponer ciertas elasticidad de sustitución entre los insumos energéticos, en este modelo se contemplan tres elasticidades de sustitución diferentes; una supone una elasticidad menor a uno que en el ámbito económico se considera como un escenario rígido, los otros dos casos se consideran flexibles al asignarles una elasticidad mayor a uno.

La primer política fiscal, supone gravar el empleo de las energías no renovables Extracción de petróleo y gas (EPG) y Fabricación de productos derivados del petróleo y del carbón (FPPC) en la producción de la energía agregada $E_{j}$; esta política considera imponer el gravamen al uso de cada una de ellas por separado o simultáneamente. La segunda política, supone que los impuestos a las energías fósiles se mantienen en el nivel definido por la economía de referencia y se asigna un subsidio al uso de energías renovables (REN). Esto implica que se debe definir un parámetro adicional que puede ser positivo o negativo, $\tau_{i j}$, dependiendo si representa una tasa impositiva o un subsidio.

Se utiliza el modelador GAMS y la interface MPSGE para obtener la solución al MCP, los datos de la SAM y los resultados obtenidos están en unidades de millones de pesos y se escriben en tablas, al incrementar los impuestos o subsidios iterativamente en un $5 \%$, desde un 5\%, hasta alcanzar un total del $70 \%$. Estos resultados se convierten a porcentajes y posibilitan construir las gráficas y cuadros que sirven para interpretar los impactos de los impuestos o subsidios en la cantidad demanda de cada bien energético, como bien intermedio. En concreto, las gráficas se interpretan al utilizar el eje de las 
abscisas para representar los incrementos a los impuestos o subsidios, en múltiplos de 10 a partir del 5\%, y el eje vertical representa el porcentaje de variación en la cantidad demanda de cada bien energético. Análogamente, las tablas utilizan los renglones para representar los porcentajes de incremento a los impuestos o subsidio, en múltiplos de 10 e iniciando con un impuesto o subsidio de 10\%; y las columnas representan el porcentaje de variación en la cantidad demanda de cada bien energético, se aclara que las interpretaciones se hacen al considerar los porcentajes como un número aproximado. Para simplificar, en adelante se utilizará el término demanda para referirse a la cantidad demandada.

\subsection{Calibración y Resultadoss}

En esta sección se describen los procesos de calibración e interpretación de resultados, bajo el supuesto de que se satisfacen las condiciones de equilibrio, descritas en el Sección 3.4, para cada uno de los agentes de la economía. La calibración consiste en determinar los valores de los parámetros, proceso requiere los datos contenidos en la matriz de contabilidad social, ver los cuadros A.1, A.2 y A.3 del Anexo A.

El análisis de los resultados proporcionan una medida de la sensibilidad de la economía a cambios de algunas variables exógenas tales como los impuestos y subsidios. En este caso, en la producción de la energía agregada se grava el uso de las energías EPG y FPPC o se subsidia el empleo del bien REN. Estas políticas se implementan en dos escenarios; el caso rígido con una elasticidad de $\sigma_{j}=0.5$ y el flexible con una elasticidad $\sigma_{j} \in\{1.8,10\}$; estas elasticidades se propusieron tomado en cuenta el Cuadro 4.1.

Cuadro 4.1: Elasticidades de sustitución técnica

\begin{tabular}{cccc} 
Sector & $\sigma$ & Fuente & País \\
\hline Energía agregada & 0.5 & $\begin{array}{c}\text { Mowers y Rausch [23] } \\
\text { Paltsev. et al. [27] }\end{array}$ & EU \\
Energía agregada & 1.8 & Saam et al. [37] & Mundial \\
Energía agregada & 10 & Saam et al. [37] & Mundial \\
\hline
\end{tabular}

Cabe aclarar que la economía se encuentra distorsionada inicialmente por los impuestos y subsidios contenidos en el equilibrio de referencia y por tanto los parámetros de calibración se calculan bajo este supuesto. 


\section{Variables de decisión}

Las variables de decisión están determinadas por las correspondientes a los modelos del productor y del consumidor, respectivamente.

- $F_{k j}$ es el factor de producción $k=L, C$ para para producir el bien $j=1, \ldots, 6$.

- $V_{j}$ valor añadido del sector $j=1, \ldots, 6$.

- $X_{i j}$ cantidad del bien intermedio $i(i=3, \ldots, 6)$ para producir una unidad del bien $Y_{j}(j=1, \ldots, 5)$.

- $I m p_{j}$ cantidad de los bienes de importaciones que se requiere producir una unidad del bien $Y_{j}(j=1, \ldots, 5)$.

- $E_{j}$ cantidad de energía agregada que se necesita para producir una unidad del bien $Y_{j}(j=1, \ldots, 5)$.

- $X_{i}$ denota la cantidad que consumen las familias del bien final $Y_{i}$, para $i=1,2,3,5$.

- $v_{j}$ precio del valor añadido $j=1, \ldots, 6$.

- $p_{j}$ precio de los bienes de producción, del consumo final y de las importaciones, para $j=1, \ldots, 6$.

- $w_{k}$ precio del factor $k=L, C$.

\section{Parámetros}

Los parámetros para cada uno de los modelos se determinan con los datos de la economía de referencia, representada en la SAM, y suponiendo que ni la tecnología de produccción ni las preferencias del consumidor cambian.

- $B_{j}$ coeficiente de escala en la función de producción del j-ésimo valor añadido.

- $\beta_{k j}$ es el coeficiente de proporcionalidad del factor $F_{k j}$, para $k=L, C$, en la función de producción del valor añadido.

- $\gamma_{i j}$ denota el coeficiente de proporcionalidad del bien intermedio $X_{i j}$ en la función de producción de la energía agregada $E_{j}$, para $i=3, \ldots, 6$ y $j=1, \ldots, 6$.

- $\rho_{j}=\frac{\sigma_{j}-1}{\sigma_{j}}$ parámetro definido por la elasticidad de sustitución.

- $\psi_{j}$ coeficiente de escala en la función de producción de la energía agregada $E_{j}$.

- $\iota_{k j}$ coeficiente de proporcionalidad del bien intermedio $X_{k j}$ en la función de producción del bien final $Y_{j}$, para $k=1,2$. 
- $\iota_{v j}, \iota_{i j}$ y $\iota_{e j}$ denotan respectivamente el coeficiente de proporcionalidad del valor añadido $V_{j}$, el coeficiente de proporcionalidad del bien intermedio de importación $I m p_{j}$ y el coeficiente de proporcionalidad de la energía agregada $E_{j}$, para producir una unidad de la producción total $Y_{j}$

- $\alpha_{i}$ coeficiente de proporcionalidad, del bien final $i=1,2,3,5$, en la función de utilidad de la familia representativa.

\subsubsection{Calibración de los parámetros}

A continuación se presenta la fase de calibración que consiste en determinar los valores de los parámetros a partir de los datos contenidos en la SAM; para llevar a cabo esta tarea se supone que los precios de los bienes intermedios, finales, importaciones, exportaciones y de los factores de producción son unitarios. El valor encontrado permite reproducir la economía, o validar el modelo. Los parámetros que se determinan son los fiscales, los coeficientes de proporcionalidad y de escala.

\section{Calibración de los parámetros}

- Parámetros fiscales

Sea $\tau_{j}$ la tasa que grava la producción total de cada industria $Y_{j}$.

De acuerdo al Cuadro A.1, para cada industria $j$ el total de impuestos a su producción corresponde a las cantidades mostradas en el renglón GOB, denotadas por $\operatorname{Tax}_{j}^{*}$, satisfacen la igualdad $\operatorname{Tax}_{j}^{*}=\tau_{j} p_{j} Y_{j}=$. Entonces la tasa para cada industria está definida como

$$
\tau_{j}=\frac{\operatorname{Tax}_{j}^{*}}{p_{j} Y_{j}}
$$

Por lo tanto el vector que contiene los valores correspondientes a la tasa que grava a la producción de las seis industrias es

$$
(0.095,0.062,-0.006,0.877,0.008,0)
$$

Sea $I v a=\tau \sum_{i=1}^{6} X_{i}$ el impuesto recaudado sobre el consumo total de la familia, en el Cuadro A.2 corresponde al renglón GOB y a la columna FAM. Entonces la tasa impositiva al consumo $\tau$ está determinada por

$$
\tau=\frac{I v a}{\sum_{i=1}^{6} X_{i}}=0.070
$$

para $i=2, \ldots, 6$ 
- Calibración de los coeficientes de proporcionalidad y de escala para el modelo del productor de bienes finales

Los coeficientes de proporcionalidad $\beta_{k j}$ y el coeficiente de escala $B_{j}$ del $j$-ésimo valor añadido se muestran en la siguiente tabla

Cuadro 4.2: Coeficientes de proporcionalidad del factor compuesto

\begin{tabular}{ccccccc} 
& SPR & ISE & GEE & EP & FPPC & REN \\
\hline$\beta_{L j}$ & 0.185 & 0.296 & 0.493 & 0.173 & 0.432 & 0.301 \\
$\beta_{C j}$ & 0.815 & 0.704 & 0.507 & 0.827 & 0.568 & 0.699 \\
& & & & & & \\
\hline$B_{j}$ & 1.615 & 1.836 & 2.000 & 1.584 & 1.981 & 1.843
\end{tabular}

Se observa que para cada $j$ los coeficientes de proporcionalidad satisfacen que $\beta_{L j}+\beta_{C j}=1$.

Para encontrar los valores de los coeficientes de proporcionalidad $\gamma_{i j}$ de las energías intermedias y coeficientes de escala $\psi_{j}$, se asume que el valor del parámetro $\sigma_{j}$ que representa la elasticidad es exógeno, para así determinar el valor de $\rho_{j}$.

Cuadro 4.3: Coeficientes de proporcionalidad y de escala en la producción de la energía agregada

\begin{tabular}{ccccccc}
$\gamma_{i j}$ & SPR & ISE & GEE & EPG & FPPC & REN \\
\hline GEE & 0.627 & 0.474 & 0.333 & 1.000 & 1.000 & 0 \\
EPG & 0 & 0.040 & 0.410 & 0 & 0 & 0 \\
FPPC & 0.373 & 0.486 & 0.050 & 0 & 0 & 0 \\
REN & 0 & 0 & 0.207 & 0 & 0 & 0 \\
\hline & & & & & & \\
$\psi_{j}$ & 1.879 & 2.161 & 3.082 & 1.000 & 1.000 & 0
\end{tabular}

- Calibración de los coeficientes de proporcionalidad para el modelo del consumidor 
Los valores de los parámetros que representan los coeficientes de proporcionalidad en la función de utilidad de la familia se obtienen a partir de la ecuación (3.26),

Cuadro 4.4: Coeficientes de proporcionalidad en la función de utilidad de la familia

\begin{tabular}{ccccccc} 
& SPR & ISE & GEE & EPG & FPPC & REN \\
\hline$\alpha_{i}$ & 0.024 & 0.946 & 0.015 & 0 & 0.015 & 0
\end{tabular}

Una vez determinados los parámetros se lleva a cabo la llamada validación del modelo, esta fase no se presenta debido a que el programa GAMS lo hace internamente.

\subsection{Resultados para el modelo Estático}

En esta sección se analizan los impactos que tienen las dos políticas económicas fiscales propuestas inicialmente, sobre las demandas de las energías como bienes intermedios en la producción de la energía agregada. En general, el mecanismo de transmisión y los efectos finales de las políticas se producen de acuerdo a los supuestos de anidamiento y de flexibilidad de sustitución entre los bienes energéticos y el resto de los insumos intermedios.

\subsubsection{Impuestos al uso de los bienes energéticos EPG y FPPC}

La primer política consiste en gravar, por separado y luego de manera conjunta, el uso de los dos bienes energéticos EPG y FPPC, en la producción de la energía agregada, para mostrar como la demanda de ambos bienes disminuye ante el incremento de los precios relativos. Se muestra qué bajo las condiciones adecuadas de sustitución técnica y al gravar simultáneamente la producción de los dos bienes energéticos EPG y FPPC, los productores de esta economía tienden a sustituirlos por las energías renovables.

\subsubsection{Escenario con rigidez en la sustitución técnica}

En general, una política impositiva en este escenario no es efectiva para lograr la sustitución de energías contaminantes por energías renovables, ya que la demanda de todas las energías desciende al aumentar sus precios relativos. Bajo estas condiciones, las empresas no sustituyen las energías fósiles por energías renovable, sino que lo hacen por los otros insumos intermedios y por capital y trabajo. Los resultados del modelo indican que la disminución es marginal pero esta tendencia permite cierto nivel de acción al decisor para reducir la contaminación debida a las emisiones de $\mathrm{CO}_{2}$. En este escenario es recomendable evaluar con precisión el efecto distorsionante del impuesto y comparar sus costos con el beneficio obtenido por la reducción en la emisión de $\mathrm{CO}_{2}$. 


\section{Impuesto al uso del bien Extracción de Petróleo y Gas (EPG)}

En el Cuadro 4.5 se observan los efectos, sobre las demandas de los energéticos, que tiene la política de gravar hasta en un $70 \%$ el precio del bien compuesto EPG en la producción de la energía agregada.

En general, las demandas de todas las energías decrecen ligeramente, incluida la demanda del propio bien EPG, así como la demanda de energía eléctrica GEE y se muestra como ambas se comportan de manera similar. Para la tasa impositiva del $70 \%$, hay una disminución de sólo $10.78 \%$ en la demanda de EPG y de $9.48 \%$ para GEE. La demanda correspondiente al bien energético FPPC permanece casi constante al disminuir solamente en $1.6 \%$, mientras que la del bien REN se reduce notoriamente al alcanzar $14.28 \%$.

Cuadro 4.5: Impuesto a la extracción de petróleo y gas

Variación de la demanda de los bienes energéticos

\begin{tabular}{ccccc}
\multicolumn{5}{c}{$(\boldsymbol{\sigma}=\mathbf{0 . 5})$} \\
Imp. \% & GEE & EPG & FPPC & REN \\
\cline { 2 - 5 } 10 & -1.720 & -2.248 & -0.277 & -2.640 \\
20 & -3.284 & -4.172 & -0.535 & -5.024 \\
30 & -4.719 & -5.840 & -0.775 & -7.193 \\
40 & -6.043 & -7.301 & -1.000 & -9.180 \\
50 & -7.270 & -8.594 & -1.211 & -11.011 \\
60 & -8.414 & -9.746 & -1.412 & -12.706 \\
70 & -9.484 & -10.780 & -1.602 & -14.282
\end{tabular}

Fuente: Elaboración propia.

Impuesto al uso del bien Fabricación de Productos Derivados del Petróleo y del Carbón (FPPC)

El Cuadro 4.6, muestra la variación de la demanda de las energías al gravar al precio del bien FPPC, globalmente hay un ligero decaimiento en las demandas de todas las energías consideradas. Por ejemplo, para una tasa impositiva de $30 \%$ la demanda del bien FPPC decrece en 10\%, y tan sólo $2 \%$ para el bien EPG. Para esta tasa sobresale el comportamiento de las demandas de los bienes energéticos GEE y REN, ya que se comportan como si fueran bienes complementarios perfectos, de manera que, la demandada del primer bien decrece en $3.8 \%$ mientras que para el segundo en $3.9 \%$, este comportamiento se presenta para cada tasa incorporada en el cuadro. 
Cuadro 4.6: Impuesto a la Fabricación de Productos Derivados del Petróleo y del Carbón Variación de la demanda de los bienes energéticos

\begin{tabular}{ccccc}
\multicolumn{5}{c}{$(\boldsymbol{\sigma}=\mathbf{0 . 5})$} \\
Imp. \% & GEE & EPG & FPPC & REN \\
\cline { 2 - 5 } 10 & -1.38 & -0.74 & -3.83 & -1.41 \\
20 & -2.65 & -1.41 & -7.13 & -2.70 \\
30 & -3.82 & -2.02 & -10.01 & -3.89 \\
40 & -4.90 & -2.57 & -12.55 & -4.98 \\
50 & -5.91 & -3.09 & -14.80 & -6.00 \\
60 & -6.85 & -3.56 & -16.81 & -6.95 \\
70 & -7.74 & -4.01 & -18.63 & -7.85
\end{tabular}

Fuente: Elaboración propia.

\section{Impuesto simultáneo a los bienes EPG y FPPC}

El porcentaje de variación de las demandas de energéticos al gravar simultáneamente el uso, en la producción de la energía agregada, de los bienes EPG y FPPC se muestra en el Cuadro 4.7. Para el caso de una tasa impositiva del $30 \%$ la demanda de estos bienes decrece en $7.6 \%$ y $10.6 \%$ respectivamente; por su parte la demanda del bien GEE se reduce en $8.3 \%$ y en $10.8 \%$ la correspondiente a la energía REN. En este caso es claro que la demanda de todos los bienes energéticos decrece, siendo mayor para el bien REN. 
Cuadro 4.7: Impuesto simultáneo a la producción de extracción de petróleo y gas y a la fabricación de productos derivados del petróleo y el carbón

Variación de la demanda de los bienes energéticos

\begin{tabular}{ccccc}
\multicolumn{5}{c}{$(\boldsymbol{\sigma}=\mathbf{0 . 5})$} \\
Imp. \% & GEE & EPG & FPPC & REN \\
\cline { 2 - 5 } 10 & -3.07 & -2.95 & -4.09 & -4.01 \\
20 & -5.83 & -5.46 & -7.59 & -7.57 \\
30 & -8.31 & -7.61 & -10.62 & -10.76 \\
40 & -10.58 & -9.48 & -13.28 & -13.64 \\
50 & -12.65 & -11.12 & -15.63 & -16.25 \\
60 & -14.56 & -12.57 & -17.72 & -18.65 \\
70 & -16.33 & -13.86 & -19.60 & -20.85
\end{tabular}

Fuente: Elaboración propia.

\subsubsection{Escenario con flexibilidad en la sustitución técnica}

En este contexto los escenarios suponen dos valores para la elasticidad de sustitución, $\sigma=1.8$ y $\sigma=10$, y los resultados alcanzados permiten concluir que la política impositiva resulta ser efectiva, ya que la economía es capaz de sustituir los insumos energéticos gravados en la producción de la energía agregada, al aumentar su precio relativo. Por lo tanto, si el objetivo consiste en menguar la utilización de las energías fósiles e inducir a que éstas se sustituyan por energías renovables para lograr una reducción de $\mathrm{CO}_{2}$ esta política es efectiva. En conclusión, la política impositiva resulta eficiente en la medida en que la economía tenga la capacidad de sustituir con mayor flexibilidad los insumos energéticos en los procesos productivos. 


\section{Impuestos al uso del bien Extracción de Petróleo y Gas (EPG)}

El Cuadro 4.8 revela que un gravamen del $20 \%$, sobre el precio del bien EPG, es suficiente para que la demandada de la energía EPG decrezca en $21 \%$ si el valor de la elasticidad de sustitución técnica es igual a 10, y en $6.8 \%$ si esta elasticidad es 1.8, y en general tiende a decrecer para cada impuesto y en cada escenario. El bien FPPC aumenta $5.3 \%$ y menos de $1 \%$ para estas elasticidades. La demanda de la energía eléctrica disminuye en $3.3 \%$, para $\sigma=1.8$, y en $0.8 \%$ en otro caso; el crecimiento de la demanda de las energías renovables se aproxima a $8.84 \%$ cuando la elasticidad es 1.8 , sin embargo es del $100 \%$ si la elasticidad es 10 .

Cuadro 4.8: Impuestos a la extracción de petróleo y gas para $\sigma=1.8,10$

Variación de la demanda de los bienes energéticos

$$
(\sigma=1.8, \sigma=10)
$$

\begin{tabular}{|c|c|c|}
\hline EPG & \multicolumn{2}{|c|}{ GEE } \\
\hline Imp. \% & 1.8 & 10 \\
\hline 10 & -1.76 & -0.88 \\
\hline 20 & -3.29 & -0.77 \\
\hline 30 & -4.63 & -0.47 \\
\hline 40 & -5.81 & -0.23 \\
\hline 50 & -6.88 & -0.08 \\
\hline 60 & -7.83 & 0.00 \\
\hline 70 & -8.70 & 0.05 \\
\hline
\end{tabular}

\begin{tabular}{cc}
\multicolumn{2}{c}{ EPG } \\
\hline $\mathbf{1 . 8}$ & $\mathbf{1 0}$ \\
\hline-3.72 & -12.62 \\
-6.76 & -20.97 \\
-9.27 & -25.73 \\
-11.38 & -28.21 \\
-13.17 & -29.46 \\
-14.71 & -30.09 \\
-16.04 & -30.42
\end{tabular}

\begin{tabular}{cc}
\multicolumn{2}{c}{ FPPC } \\
\hline $\mathbf{1 . 8}$ & $\mathbf{1 0}$ \\
\hline 0.39 & 3.36 \\
0.74 & 5.33 \\
1.05 & 6.40 \\
1.33 & 6.95 \\
1.58 & 7.24 \\
1.82 & 7.40 \\
2.03 & 7.48
\end{tabular}

\begin{tabular}{cc}
\multicolumn{2}{c}{ REN } \\
\hline $\mathbf{1 . 8}$ & $\mathbf{1 0}$ \\
\hline 4.59 & 54.15 \\
8.84 & 99.80 \\
12.82 & 130.29 \\
16.53 & 148.10 \\
20.02 & 157.92 \\
23.31 & 163.29 \\
26.41 & 166.26
\end{tabular}

Fuente: Elaboración propia

Para la máxima tasa impositiva, $70 \%$, la demanda de la energía de origen fósil FPPC apenas se eleva en $2 \%$ cuando el valor de la elasticidad de sustitución es de 1.8 y aumenta ligeramente hasta $7.5 \%$ para el escenario de mayor flexibilidad. Por otra parte, la demanda del bien energético GEE, disminuye en $8.7 \%$ cuando la elasticidad es de 1.8 , no obstante la demanda del bien energético renovable aumenta en $26.4 \%$. Si la elasticidad aumenta hasta 10 la demanda de la energía eléctrica no se incrementa sustancialmente, $5 \%$, pero la del bien renovable REN se incrementa $166.25 \%$.

En general, el comportamiento cualitativo se puede apreciar en las gráficas 4.1.a y 4.1.b de la Fig. 4.1, se observa que la demanda de los bienes EPG y GEE decrece para ambos escenarios siendo más notorio este descenso para $\sigma=1.8$; en contraste la demanda del bien REN es notablemente superior para $\sigma=10$ que para $\sigma=1.8$. 


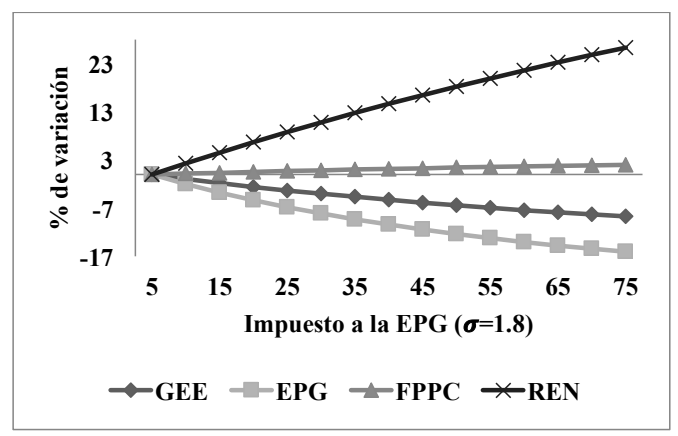

Fig. 4.1.a

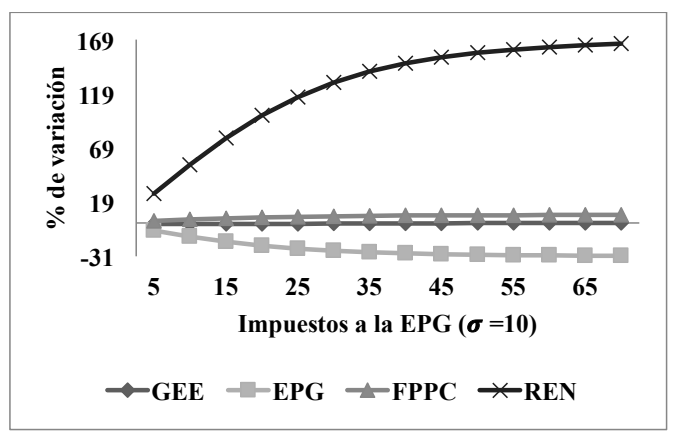

Fig. 4.1.b

Figura 4.1: Gráfica de las demandas de las energías bajo impuestos a la extracción de petróleo y gas, para $\sigma=1.8,10$

Fuente: Elaboración propia.

Impuesto al uso del bien Fabricación de Productos Derivados del Petróleo y del Carbón (FPPC)

En la Fig. 4.2, las gráficas 4.2.a y 4.2.b exhiben que para ambos escenarios las demandas de los bienes energéticos REN y GEE se comportan de manera muy semejante. Ciertamente la correspondiente al bien FPPC decrece, pero en contraste la demanda del bien fósil EPG es creciente.

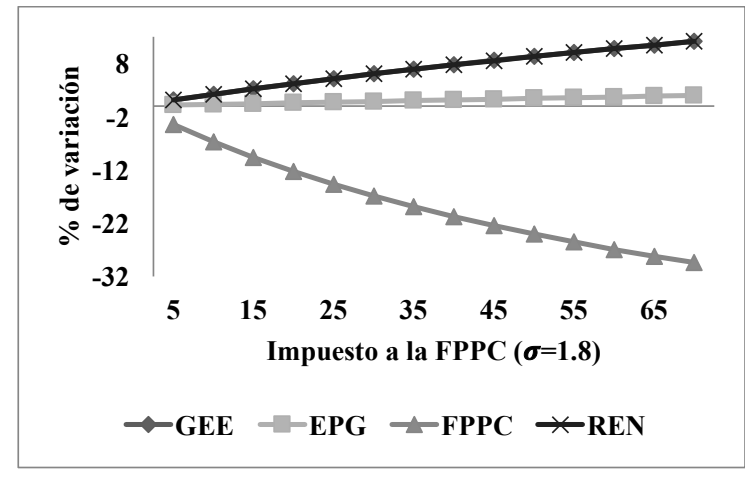

Fig. 4.2.a

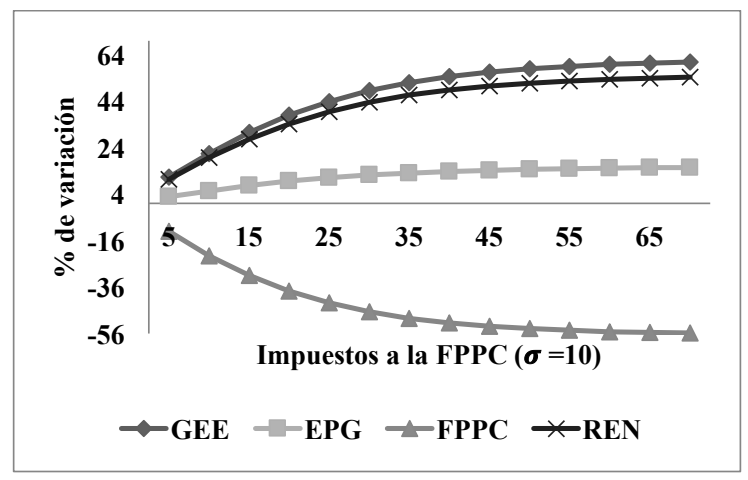

Fig. 4.2.b

Figura 4.2: Gráfica de las demandas de las energías bajo impuestos a la fabricación de productos derivados del petróleo y el carbón, para $\sigma=1.8,10$

Fuente: Elaboración propia. 
El Cuadro 4.9 señala que para cada impuesto sobre el precio de los derivados del petróleo y del carbón y para cada escenario hay un descenso en la demanda de este bien. El estudio del comportamiento de la demanda del resto de los energéticos se toma un gravamen del $70 \%$; así la demandada del energético EPG se incrementa en $15.4 \%$ cuando la elasticidad es 10 y solamente $1.9 \%$ si la elasticidad es 1.8, la demanda de la energía GEE aumenta aproximadamente $12.1 \%$ si el valor de la elasticidad es 1.8 y en $60 \%$ si el valor correspondiente es 10. Por último, la demanda del bien renovable se eleva en $54.3 \%$ y $12.1 \%$, cuando los valores de la elasticidad son $\sigma=10$ y $\sigma=1.8$, respectivamente.

Cuadro 4.9: Impuestos a la fabricación de productos derivados del petróleo y el carbón, para $\sigma=1.8,10$

Variación de la demanda de los bienes energéticos

$$
(\sigma=1.8, \sigma=10)
$$

\begin{tabular}{|c|c|c|c|c|c|c|c|c|}
\hline \multirow{2}{*}{$\begin{array}{l}\text { FPPC } \\
\text { Imp. } \%\end{array}$} & \multicolumn{2}{|c|}{ GEE } & \multicolumn{2}{|c|}{ EPG } & \multicolumn{2}{|c|}{ FPPC } & \multicolumn{2}{|c|}{ REN } \\
\hline & 1.8 & 10 & 1.8 & 10 & 1.8 & 10 & 1.8 & 10 \\
\hline 10 & 2.20 & 21.50 & 0.27 & 5.38 & -6.76 & -22.46 & 2.17 & 19.60 \\
\hline 20 & 4.20 & 37.89 & 0.55 & 9.51 & -12.30 & -37.72 & 4.16 & 34.18 \\
\hline 30 & 6.04 & 48.44 & 0.84 & 12.20 & -16.91 & -46.66 & 5.99 & 43.47 \\
\hline 40 & 7.73 & 54.55 & 1.12 & 13.76 & -20.80 & -51.44 & 7.69 & 48.82 \\
\hline 50 & 9.29 & 57.91 & 1.40 & 14.64 & -24.11 & -53.88 & 9.26 & 51.78 \\
\hline 60 & 10.75 & 59.74 & 1.68 & 15.12 & -26.95 & -55.13 & 10.73 & 53.40 \\
\hline 70 & 12.10 & 60.75 & 1.95 & 15.39 & -29.42 & -55.77 & 12.10 & 54.30 \\
\hline
\end{tabular}

Fuente: Elaboración propia.

\section{Impuesto simultáneo al uso de los bienes EPG y FPPC}

Para el caso en que la política impositiva consiste en gravar simultáneamente el uso de los bienes EPG y FPPC, la demanda de ambos disminuye, en contraste las demandas de la generación de electricidad y de la energía renovable se elevan. Por lo tanto y a pesar de que cualquier impuesto distorsiona la economía, es más recomendable establecer una política impositiva que grave a ambos bienes de manera conjunta, ya que así se promueve la sustitución de energías fósiles por renovables. 
En el Cuadro 4.10 se observan de manera precisa los efectos que tiene la política de gravar simultáneamente el precio de los bienes EPG y FPPC sobre las demandas de energéticos. En general, los impactos en la demanda son mayores en relación a los obtenidos cuando el gravamen se aplica de manera independiente a cada uno de estos bienes energéticos. La demanda de las energías fósiles, EPG y FPPC, decrecen a mayor tasa mientras que la correspondiente a las energías eléctrica y renovables aumenta tambien a mayor tasa.

Cuadro 4.10: Impuestos simultáneos a los bienes energéticos EPG y FPPC, para $\sigma=$ $1.8 ; \sigma=10$

Variación de la demanda de los bienes energéticos

$(\sigma=1.8, \sigma=10)$

\begin{tabular}{ccc} 
EPG y FPPC & \multicolumn{2}{c}{ GEE } \\
\cline { 2 - 3 } Imp. \% & $\mathbf{1 . 8}$ & $\mathbf{1 0}$ \\
\cline { 2 - 3 } 10 & 0.41 & 21.66 \\
20 & 0.81 & 41.35 \\
30 & 1.19 & 56.02 \\
40 & 1.57 & 65.41 \\
50 & 1.93 & 70.92 \\
60 & 2.28 & 74.03 \\
70 & 2.62 & 75.79
\end{tabular}

\begin{tabular}{cc}
\multicolumn{2}{c}{ EPG } \\
\hline $\mathbf{1 . 8}$ & $\mathbf{1 0}$ \\
\hline-3.54 & -9.74 \\
-6.50 & -18.93 \\
-9.00 & -25.48 \\
-11.15 & -29.38 \\
-13.02 & -31.50 \\
-14.65 & -32.62 \\
-16.08 & -33.21
\end{tabular}

\begin{tabular}{cc}
\multicolumn{2}{c}{ FPPC } \\
\hline $\mathbf{1 . 8}$ & $\mathbf{1 0}$ \\
\hline-6.40 & -19.61 \\
-11.68 & -34.49 \\
-16.10 & -44.27 \\
-19.84 & -49.97 \\
-23.05 & -53.06 \\
-25.82 & -54.69 \\
-28.23 & -55.55
\end{tabular}

\begin{tabular}{cc}
\multicolumn{2}{c}{ REN } \\
\hline $\mathbf{1 . 8}$ & $\mathbf{1 0}$ \\
\hline 6.87 & 86.10 \\
13.42 & 175.82 \\
19.68 & 246.08 \\
25.67 & 291.71 \\
31.41 & 318.60 \\
36.92 & 333.87 \\
42.21 & 342.50
\end{tabular}

Fuente: Elaboración propia.

Por ejemplo, las demandas de las energías de origen fósil decrecen de forma más notoria, para una tasa impositiva de $20 \%$ sobre los precios de los bienes energéticos EPG y FPPC, se decrementa la demanda de los bienes EPG y FPPC en $18.9 \%$ y $34.5 \%$ respectivamente, si el valor de la elasticidad es 10; mientras que solamente disminuyen en $6.5 \%$ y $11.7 \%$ cuando la elasticidad tiene un valor de 1.8. Para esta misma elasticidad la demanda de la energía eléctrica se incrementa solamente $0.8 \%$, mientras que la del bien REN lo hace en 13.4\%. En cambio, si la elasticidad es de 10 la demanda del bien GEE crece en $41.4 \%$, lo que ocasiona un aumento significativo en la demanda de las energías renovables, $175.8 \%$. 
Las gráficas 4.3.a y 4.3.b de la Fig. 4.3, muestran el impacto cualitativo sobre las demandas de cada uno de los energéticos cuando se ejerce esta política.

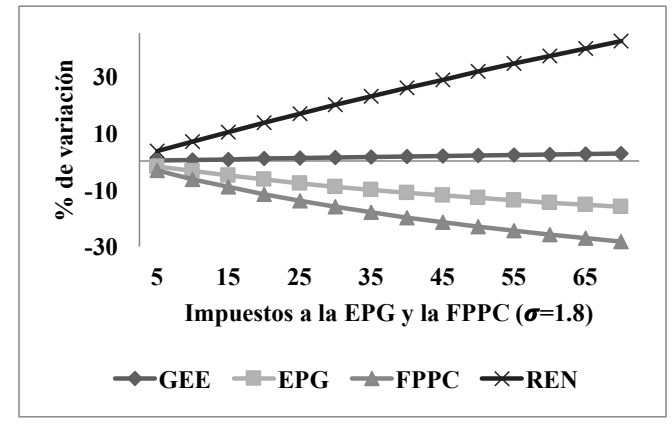

Fig. 4.3.a

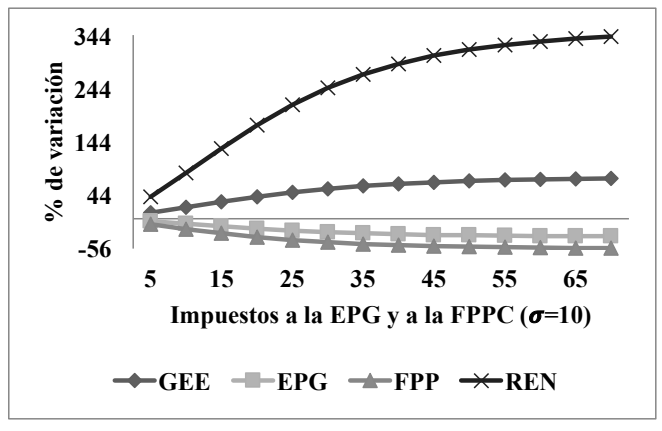

Fig. 4.3.b

Figura 4.3: Gráfica de las demandas de las energías bajo impuestos simultáneos a la EPG y la FPPC, para $\sigma=1.8,10$

Fuente: Elaboración propia.

\subsubsection{Subsidio al uso de las energías renovables REN}

Los resultados obtenidos al otorgar un subsidio al uso del bien renovable son similares a los generados bajo una política de impuestos, en el sentido de que la efectividad de esta política depende de que exista una mayor elasticidad de sustitución técnica entre las energías.

\subsubsection{Escenario con rigidez en la sustitución técnica}

Si la política consiste en otorgar un subsidio al uso del bien energético renovable las energías GEE, EPG y FPPC crecen de manera insignificante, es decir, no se logra una sustitución representativa de las energías fósiles por energías renovables. Sin embargo, existe el beneficio de producir una mayor cantidad de energía renovable, a diferencia de la política impositiva. 
La Fig. 4.4, muestra el comportamiento cualitativo de las demandas de las energías intermedias al aplicar un subsidio de hasta $70 \%$ al uso de las energías renovables, para este impuesto la demanda de este bien ergético crece cerca del 100\% (Gráfica 4.4.a, Fig. 4.4) mientras que la demanda de los otros bienes energéticos se queda entre 0 y $5 \%$ (Gráfica 4.4.b, Fig. 4.4).

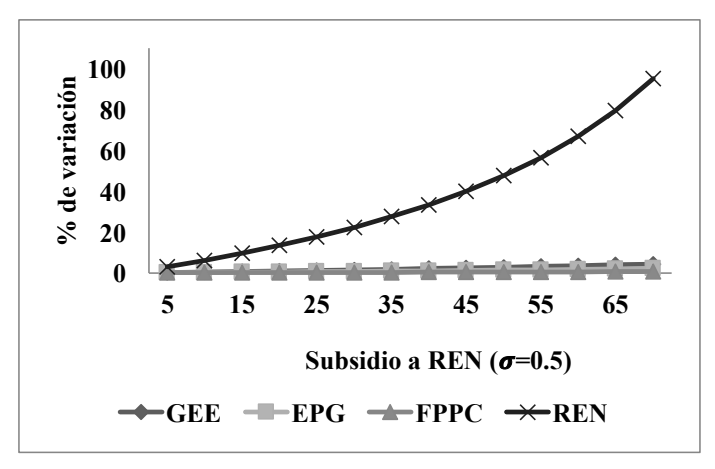

Fig. 4.4.a

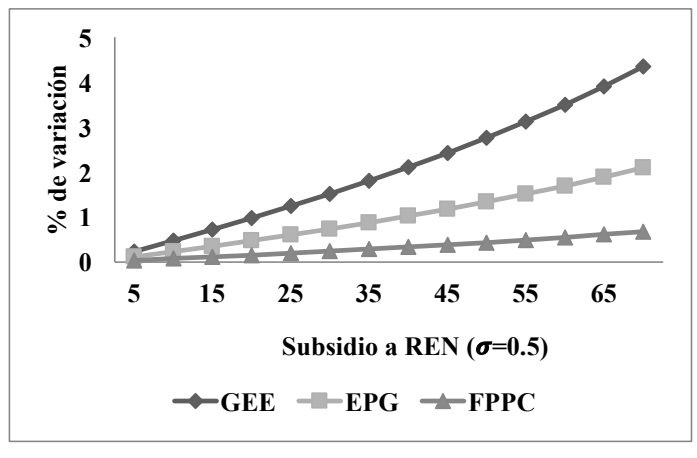

Fig. 4.4.b

Figura 4.4: Gráfica de las demandas de las energías bajo asignación de subsidios al uso de las energías renovables, para $\sigma=0.5$

Fuente: Elaboración propia.

A diferencia del uso de una política impositiva, el Cuadro 4.11 indica que las demandas de las energías EPG y FPPC, permanecen casi constantes, lo que significa que no hay sustitución significativa entre los bienes energéticos. La demanda del bien energético GEE asciende ligeramente, por ejemplo, ante una tasa de subsidio del $30 \%$ ocurre un aumento de $1.5 \%$, mientras que la demanda del bien renovable aumenta aproximadamente $22.3 \%$ para esta tasa. La demanda de las energías de origen fósil EPG y FPPC crecen a lo más en $2.1 \%$ y $0.7 \%$ respectivamente; la energía eléctrica no llega a una tasa del $5 \%$ y la correspondiente a las energías renovables crece de forma significativa. 
Cuadro 4.11: Subsidios al uso de las energías renovables Variación de la demanda de los bienes energéticos

\begin{tabular}{ccccc}
\multicolumn{5}{c}{$(\boldsymbol{\sigma}=\mathbf{0 . 5})$} \\
Subs. \% & GEE & EPG & FPPC & REN \\
\cline { 2 - 5 } 10 & 0.47 & 0.23 & 0.07 & 6.18 \\
20 & 0.97 & 0.47 & 0.15 & 13.49 \\
30 & 1.52 & 0.73 & 0.24 & 22.33 \\
40 & 2.11 & 1.02 & 0.33 & 33.33 \\
50 & 2.76 & 1.33 & 0.43 & 47.50 \\
60 & 3.50 & 1.69 & 0.54 & 66.74 \\
70 & 4.35 & 2.09 & 0.68 & 94.98
\end{tabular}

Fuente: elaboración propia.

\subsubsection{Subsidios en una economía con flexibilidad en la sustitución técnica}

Cuando la economía permite la sustitución entre los bienes energéticos como insumos en la función de producción de la energía agregada, los resultados de las demandas de los bienes energéticos difieren del caso rígido, como se observa en el Cuadro 4.12. Los datos del cuadro permiten concluir que a mayor subsidio al empleo de energía renovable, mayor es la tasa de decrecimiento en la demanda de las energías de origen fósil EPG y FPPC; esta tendencia se acentúa a medida que la sustitución técnica presenta mayor elasticidad. 
Cuadro 4.12: Subsidios al uso de las energías renovables $(\sigma=1.8 ; \sigma=10)$

Variación de la demanda de los bienes energéticos

\begin{tabular}{|c|c|c|c|c|c|c|c|c|}
\hline \multirow[b]{3}{*}{ Subs. \% } & \multicolumn{6}{|c|}{$(\sigma=1.8, \sigma=10)$} & & \\
\hline & \multicolumn{2}{|c|}{ GEE } & \multicolumn{2}{|c|}{ EPG } & \multicolumn{2}{|c|}{ FPPC } & \multicolumn{2}{|c|}{ REN } \\
\hline & 1.8 & 10 & 1.8 & 10 & 1.8 & 10 & 1.8 & 10 \\
\hline 10 & 0.52 & 0.66 & -0.42 & -6.01 & -0.11 & -1.43 & 19.28 & 131.68 \\
\hline 20 & 1.15 & 2.79 & -0.91 & -15.25 & -0.23 & -4.50 & 45.10 & 379.97 \\
\hline 30 & 1.92 & 8.39 & -1.52 & -24.37 & -0.38 & -10.08 & 80.91 & 727.97 \\
\hline 40 & 2.90 & 19.13 & -2.27 & -29.95 & -0.57 & -18.16 & 132.84 & 1124.18 \\
\hline 50 & 4.21 & 35.11 & -3.24 & -32.48 & -0.82 & -27.80 & 212.77 & 1584.96 \\
\hline 60 & 6.02 & 55.67 & -4.56 & -33.72 & -1.15 & -37.60 & 346.40 & 2198.87 \\
\hline 70 & 8.75 & 80.66 & -6.48 & -34.67 & -1.61 & -45.98 & 599.32 & 3137.69 \\
\hline
\end{tabular}

Fuente: elaboración propia.

En el caso particular en que la tasa de subsidio al bien REN es $30 \%$ y la elasticidad de sustitución es igual a 1.8, las demandas de los bienes energéticos EPG y FPPC disminuyen de forma casi nula, $1.5 \%$ y $0.4 \%$ respectivamente, pero decrecen en $24.4 \%$ y $10.1 \%$ si la elasticidad de sustitución es igual a 10. La demanda del bien GEE se incrementa en $1.9 \%$ y en contraste, la demanda del bien REN lo hace en $80.9 \%$, si la elasticidad de sustitución es 1.8. La demanda de bien energético GEE crece en $8.4 \%$, mientras la del bien energético REN aumenta aproximadamente en $728 \%$ cuando la elasticidad es 10. En general, se observa un crecimiento notable de las demandas de las energías eléctrica y renovable en este escenario.

Las gráficas 4.5.a y 4.6.a muestran el comportamiento cualitativo global de la demanda de todas las energías en los escenarios en que las elasticidades asumen los valores de 1.8 y 10, análogamente las gráficas 4.5.b 4.6.b revelan la pauta que sigue la demanda de las energías de origen fósil y de la eléctrica para estos mismos escenarios. 


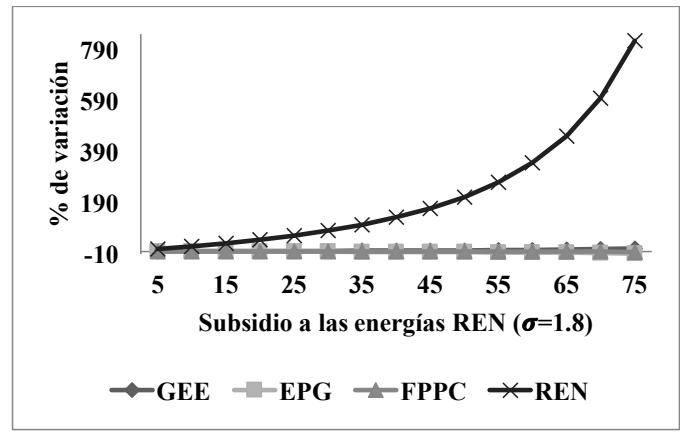

Fig. 4.5.a

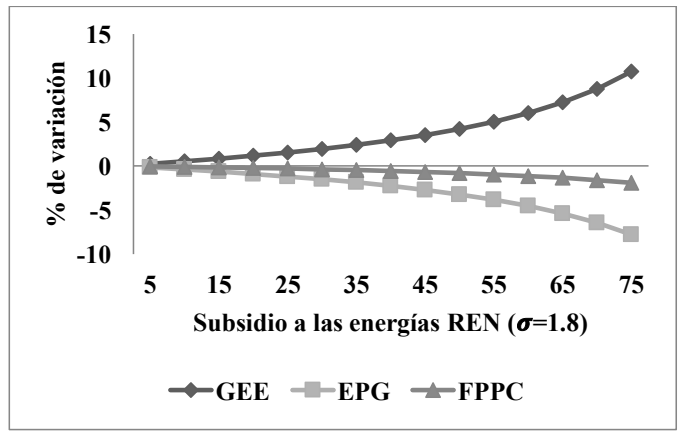

Fig. 4.5.b

Figura 4.5: Comparativo de las demandas de energías bajo asignación de subsidios al uso de las energías renovables $\sigma=1.8$

Fuente: Elaboración propia.

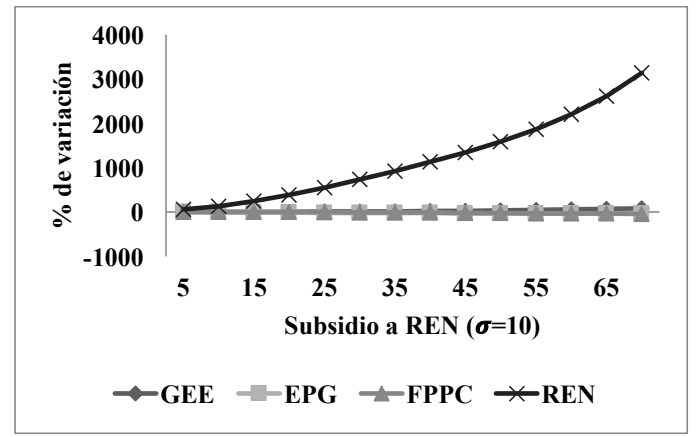

Fig. 4.6.a

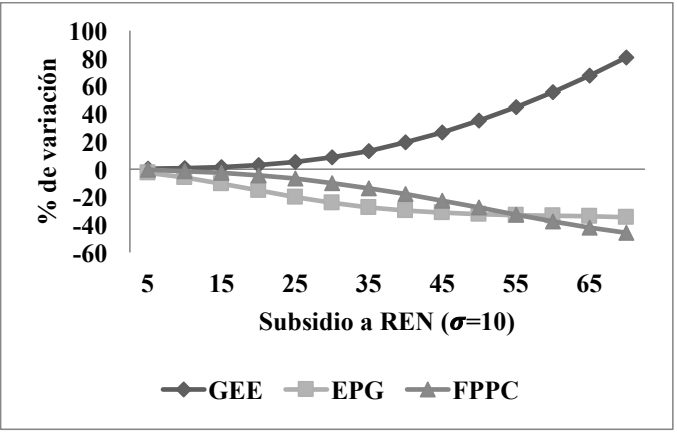

Fig. 4.6.b

Figura 4.6: Comparativo de las demandas de energías bajo asignación de subsidios al uso de las energías renovables $\sigma=10$

Fuente: Elaboración propia.

Bajo este escenario de flexibilidad, si el objetivo del gobierno es disminuir el uso de las energías de origen fósil y sustituirlas por energías renovables, sin que la economía sufra los efectos distorsionantes surgidos por la aplicación de los impuestos a las energías fósiles, entonces es recomendable asignar subsidios al uso del bien renovable. 


\section{5 Modelo Dinámico}

Los MEGC dinámicos deben involucrar condiciones macroeconómicas, como se mencionó en el Capítulo 2, se pueden plantear utilizando modelos de Ramsey, de Solow y de Generaciones traslapadas, entre otros. Estos modelos se pueden desarrollar de manera determinista o bien estocástica, discretos o continuos, y con un horizonte finito o infinito. Los modelos de Ramsey se utilizan para deteminar las fluctuaciones económicas causadas por cambios en la oferta y/o demanda, para modelos de equilibrio en el que se persigue optimizar la función de utilidad de un consumidor representativo; por su parte los modelos de Solow son muy recurridos en teoría del crecimiento, o bien se pueden utilizar para determinar cómo se ven afectadas las finanzas públicas cuando se modifica o se instaura un impuesto (enduring tax cut), o se recortan algunos presupuestos. Finalmente, los modelos de Generaciones traslapadas, a diferencia de los modelos de Ramsey y Solow, generalmente se utilizan cuando existen agentes heterogéneos con vida finita, esto es para medir la distribución de ingresos, la asignación de precios a los activos, así como en las finanzas públicas. Por las caracterśticas del objetivo que se persigue en este trabajo se utiliza un modelo tipo Ramsey.

\subsection{Descripción e hipótesis del modelo dinámico}

El modelo Ramsey es un modelo clásico de la macroeconomía que se usa frecuentemente en los MEGC dinámicos que también determinan las variables que definen el llamado producto interno bruto PIB, sin olvidar que permite optimizar la función de utilidad del consumidor representativo en cada periodo.

Las hipótesis generales del modelo estático se mantienen y las correspondientes al modelo dinámico se describen a continuación. El modelo Ramsey que se utiliza en este 
trabajo es determinista, el tiempo se considera una variable discreta y se supone un horizonte de planeación infinito. Estos modelos aseguran que se maximiza la utilidad obtenida por la familia representativa para cada periodo de tiempo $t$. Los agentes, como en el modelo estático, son un consumidor que tiene una vida infinita, seis productores y el gobierno. Los modelos tipo Ramsey en el contexto de la optimización están representados como modelos de Programción no Lineal, y por su uso en los modelos de equilibrio son analizados en el marco de los modelos complementarios mixtos, que resultan útiles, ya que se les pueden incorporar políticas de ahorro e inversión, condiciones que requieren los modelos de equilibrio dinámico. En cada periodo $t$ cada uno de los agentes, la familia representativa, el productor y el gobierno, se comportan como en el caso estático; y cada industria produce a través de un proceso anidado.

Generalmente, un modelo con horizonte infinito se puede abordar de dos formas: una es utilizando las ecuaciones de Euler que definen un conjunto de ecuaciones en diferencias; la segunda forma es a través del planteamiento de una recursividad que se resuelve con programación dinámica y cuya solución determina una función de capital que permite determinar el nivel de consumo de los agentes. En cualquiera de los dos casos, el horizonte infinito se divide en dos espectros temporales, uno que involucra los primeros $t=1, \ldots, T$ periodos y el segundo que comprende el resto de los periodos. Debido a que no se puede asegurar que las aproximaciones se encuentren en la llamada trayectoria estable, para obtener una buena aproximación se utilizan restricciones sobre el capital acumulado para el periodo $T+1$.

A continuación se desarrolla el modelo, que a diferencia del caso estático, requiere condiciones de tipo macroeconómico que incluyen: condiciones iniciales sobre el capital, condiciones de crecimiento del trabajo, y condiciones de cerradura que conduzcan a la solución a un estado estacionario a partir del intervalo de tiempo $t=T$, ya que el modelo utiliza un horizonte de planeación infinito. Para cada intervalo de tiempo se definen y describen las condiciones de equilibrio a ser satisfechas, algunas coinciden con las asociadas al caso estático.

\subsection{Modelos}

En este contexto los modelos tipo Ramsey pueden ser resueltos utilizando dos problemas de condiciones complementarias mixtas, [22]. El primer problema se puede interpretar como las condiciones de primer orden de un problema de programación no lineal; el segundo corresponde a las condiciones de equilibrio. Las preferencias y tecnologías se representan por medio de funciones de utilidad y producción, respectivamente, en el problema primal y en el dual se representan a través de las funciones de gasto y de costos respectivamente.

Los modelos correspondientes para el productor y el consumidor se tienen que resolver simultáneamente en términos de un sistema de ecuaciones complementarias, de 
manera conjunta con las condiciones de equilibrio para cada periodo $t$. Por su parte, el gobierno se modela como un recaudador de impuestos. Se omite la representación del modelo correspondiente al productor, debido a que para cada periodo de tiempo se resuelve el correspondiente al modelo estático; sin embargo el modelo del consumidor se presenta para describir como está involucrado su dual y como se puede representar a través de dos problemas, uno de ellos con horizonte finito.

\subsubsection{Modelo para el consumidor}

Las restricciones del modelo del consumidor, en este caso, también dependen del capital inicial, que está determinado por la dotación inicial del capital de la familia y se denota por $F_{C}^{0}=F_{C}$, y del crecimiento del trabajo, el cual se supone crece a una tasa constante $g$ a lo largo del horizonte. En este modelo, como en el caso estático, se mantienen las transferencias realizadas por el gobierno (por medio de programas sociales) y por el sector externo (como las remesas).

Por lo tanto, el modelo para el consumidor representativo consiste en:

$$
\max U\left(X_{1}^{t}, \ldots, X_{5}^{t}\right)=\sum_{t=0}^{\infty}\left(\frac{1}{1+\delta}\right)^{t} \Pi_{i=1, i \neq 4}^{5}\left(X_{i}^{t}\right)^{\alpha_{i}}
$$

Sujeto a:

$$
\sum_{t=0}^{\infty}\left[(1+\tau) \sum_{i=1}^{5} p_{i}^{t} X_{i}^{t}+A_{p}^{t}\right]=w_{C}^{0} F_{C}^{0}+\sum_{t=0}^{\infty}\left(w_{L}^{t} F_{L}^{t}+T_{g}^{t}+T_{e}^{t}\right)
$$

donde $\sum_{i=1}^{5} \alpha_{i}=1$ y $\alpha_{i} \in[0,1]$, y $F_{C}^{0}=F_{C}$.

\section{Notación}

- $p_{i}^{t}$ son los precios de los bienes de consumo final en el periodo $t$.

- $\alpha_{i}$ representa el coeficiente de participación, del bien final $i=1, \ldots, 5$, en la función de utilidad de la familia.

- $A_{p}^{t}$ es el ahorro de la familia en el periodo $t$.

- $F_{k}^{t}$ denota la dotación del factor $k=L, C$ de la familia representativa en el periodo $t$.

- $w_{k}^{t}$ es el costo del factor $k=L, C$ en el periodo $t$.

- $\tau$ define la tasa que grava el consumo total de la familia.

- $\delta$ es la tasa de depreciación del capital 
- $T_{g}^{t}$ son las transferencias del gobierno a la familia en el periodo $t$.

- $T_{e}^{t}$ denotan las transferencias del sector externo a la familia en el periodo $t$.

\subsubsection{Condiciones intertemporales e iniciales}

Las condiciones macroeconómicas son intertemporales y determinan el crecimiento de la economía; estas condiciones están relacionadas con el consumo, producción e inversión agregadas. En una economía cerrada sin sector gobierno, ver [38], el consumo agregado $X^{t}$ está determinado por la producción agregada $Y^{t}$ menos la inversión agregada $I^{t}, X^{t}=Y^{t}-I^{t}$; para este modelo se tiene una economía abierta con un sector gobierno. Entonces, esta identidad debe considerar el consumo del gobierno $X_{g}^{t}$ y la balanza comercial $I m p^{t}-E x p^{t}$, por lo que se expresa como:

$$
X^{t}=Y^{t}-I^{t}-X_{g}^{t}+\left(I m p^{t}-E x p^{t}\right)
$$

En el caso de los modelos dinámicos el crecimiento del capital $F_{C}^{t+1}$ para cada periodo $t+1$ depende del valor del capital $(1-\delta) F_{C}^{t}$ en el periodo $t$ más la inversión agregada correspondientes $I^{t}$, donde $\delta$ es la tasa de depreciación del capital. Esta condición se debe satisfacer en cada periodo $t+1$ y se integra al modelo mediante la ecuación:

$$
F_{C}^{t+1}=(1-\delta) F_{C}^{t}+I^{t}
$$

Para el caso particular en que $t=0$ el valor inicial del capital se definió en la Sección 5.2.1 como $F_{C}^{0}=F_{C}$, y a inversión inicial se obtiene como la suma de las inversiones iniciales de cada sector, representadas en la SAM (Ver la columna AHO del Cuadro 8.3), más la transferencia del ahorro al sector externo:

$$
I^{0}=\sum_{j=1}^{5} \operatorname{In} v_{j}^{0}+T A_{e}
$$

Para determinar el factor trabajo en cada periodo $t$ se requiere conocer el trabajo inicial $F_{L}^{0}$ que corresponde la dotación de la familia $F_{L}$ definida por la SAM, ver el Cuadro 8.2 en el renglón FAM y la columna LB, por lo que $F_{L}^{0}=F_{L}$ Respecto al crecimiento del trabajo en la literatura existen diferentes formas de crecimiento, aquí se supone que crece a una tasa constante $g$. En general, el crecimiento del trabajo para cada periodo de tiempo está determinado por: 


$$
F_{L}^{t}=F_{L}^{0}(1+g)^{t}
$$

o de manera equivalente

$$
F_{L}^{t}=(1+g) F_{L}^{t-1}
$$

\subsection{Modelos Complementarios}

En esta sección se describe la forma de obtener la solución al utilizar las condiciones complementarias, que resultan ser las condiciones de holgura complementaria para el caso de los modelos primal y dual no lineales. Estas condiciones también surgen en modelos del cálculo variacional, y no necesariamente en un contexto de optimización, (Ver [35] y [18]).

Si el modelo del consumidor se considera como un modelo primal, entonces su dual consiste en minimizar la llamada función de gasto, y está determinado por

$$
\min \sum_{t=0}^{\infty}\left[(1+\tau) \sum_{i=1}^{5} p_{i}^{t} X_{i}^{t}+A_{p}^{t}\right]
$$

Sujeto a:

$$
U\left(X_{1}^{t}, \ldots, X_{5}^{t}\right)=\sum_{t=0}^{\infty}\left(\frac{1}{1+\delta}\right)^{t} \Pi_{i=1, i \neq 4}^{5}\left(X_{i}^{t}\right)^{\alpha_{i}}=1
$$

\subsubsection{Problema complementario mixto}

Para determinar el equilibrio se utilizan las condiciones complementarias, las cuales están integradas por un sistema de igualdades y desigualdades, que relacionan restricciones del problema primal (dual) y variables duales (primales), siempre y cuando el problema contenga modelos de optimización.

Condiciones de cero beneficio

Las condiciones de equilibrio que se presentaron en la Sección 3.4 siguen siendo válidas para cada periodo $t$ y para cada uno de los modelos, sin embargo existen condiciones adicionales relacionadas con los aspectos macroeconómicos que deben incluirse y las condiciones de ingresos balanceados para la familia deben ser modificadas.

Las condiciones de equilibrio macroeconómicas que deben integrarse involucran las siguientes variables agregadas para cada periodo $t$ : la inversión $I^{t}$, la producción $Y^{t}$, el consumo $C^{t}$ y el capital $F_{C}^{t}$. Asociados a la variable $Y^{t}$ están su precio $p^{t}$ y su costo $c^{t}$; y la variable $F_{C}^{t}$ tiene asignado su precio $P_{C}^{t}$ y la renta $w_{C}^{t}$ del mismo. Entonces las 
condiciones de equilibrio que deben satisfacer estas variables y sus precios se establecen como:

\begin{tabular}{ccc}
$\begin{array}{c}\text { Restricciones } \\
\text { duales }\end{array}$ & $\begin{array}{c}\text { Variables } \\
\text { primales }\end{array}$ & $\begin{array}{c}\text { Condiciones } \\
\text { Complementarias }\end{array}$ \\
\hline$p^{t} \geq P_{C}^{t+1}$ & $I^{t} \geq 0$ & $I^{t}\left[p^{t}-P_{C}^{t+1}\right]=0$ \\
\hline$P_{C}^{t} \geq w_{C}^{t+1}+(1-\delta) P_{C}^{t+1}$ & $F_{C}^{t} \geq 0$ & $P_{C}^{t} \geq\left[w_{C}^{t+1}+(1-\delta) P_{C}^{t+1}\right] F_{C}^{t}=0$ \\
\hline$c^{t} \geq p^{t}$ & $Y^{t} \geq 0$ & $Y^{t}\left(c^{t}-p^{t}\right)=0$
\end{tabular}

Condiciones de vaciado de mercados

\begin{tabular}{ccc}
\hline $\begin{array}{c}\text { Restricciones } \\
\text { duales }\end{array}$ & $\begin{array}{c}\text { Variables } \\
\text { duales }\end{array}$ & $\begin{array}{c}\text { Condiciones } \\
\text { Complementarias }\end{array}$ \\
\hline$Y_{i}^{t} \geq X_{i}^{t}\left(p_{i}^{t}, M\right)$ & $p_{i}^{t} \geq 0$ & $p_{i}^{t}\left(Y_{i}^{t}-X_{i}^{t}\left(p_{i}^{t}, M\right)\right)=0$ \\
\hline$V_{t}^{j} \geq Y_{j}^{t} \frac{\partial C_{j}^{t}\left(v_{j}^{t}, p_{1}^{t}, p_{2}^{t}, e_{j}^{t}\right)}{\partial v_{j}^{t}}$ & $v_{j}^{t} \geq 0$ & $v_{j}^{t}\left(V_{j}^{t}-Y_{j}^{t} \frac{\partial C_{t}^{t}\left(v_{j}^{t}, p_{1}^{t}, p_{2}^{t}, e_{j}^{t}\right)}{\left.\partial v_{j}^{t}\right)=0}\right.$ \\
\hline$X_{i j}^{t} \geq Y_{j}^{t} \frac{\partial C_{j}^{t}\left(v_{j}^{t}, p_{1}^{t}, p_{2}^{t}, e_{j}^{t}\right)}{\partial p_{j}^{t}}$ & $p_{i}^{t} \geq 0$ & $p_{i}^{t}\left(X_{i j}^{t}-Y_{j}^{t} \frac{\partial C_{j}^{t}\left(v_{j}^{t}, p_{1}^{t}, p_{2}^{t}, e_{j}^{t}, p_{i}^{t}\right)}{\left.\partial p_{t}^{t}\right)=0}\right.$ \\
\hline$E_{j}^{t} \geq Y_{j}^{t} \frac{\partial C_{j}^{t}\left(v_{j}^{t}, p_{1}^{t}, p_{2}^{t}, e_{j}^{t}\right)}{\partial e_{t}^{t}}$ & $e_{j}^{t} \geq 0$ & $e_{j}^{t}\left(E_{j}^{t}-Y_{j}^{t} \frac{\partial C_{j}^{t}\left(v_{j}^{t}, p_{1}^{t}, p_{2}^{t}, e_{j}^{t}\right)}{\left.\partial e_{j}^{t}\right)=0}\right.$ \\
\hline$I m p_{j}^{t} \geq Y_{j}^{t} \frac{\partial C_{j}^{t}\left(v_{j}^{t}, p_{1}^{t}, p_{2}^{t}, e_{j}^{t}\right)}{\partial p_{t}^{t}}$ & $p_{j}^{t} \geq 0$ & $p_{j}^{t}\left(I m p_{j}^{t}-Y_{j}^{t} \frac{\partial C_{j}^{t}\left(v_{j}^{t}, p_{1}^{t}, p_{2}^{t}, e_{j}^{t}\right)}{\left.\partial p_{j}^{t}\right)=0}\right.$ \\
\hline
\end{tabular}

En donde $X_{i}^{t}=X_{i}^{t}\left(p_{i}^{t}, M\right)$ representa la demanda de los bienes de consumo de las familias, como función de los precios de los bienes y de los ingresos de ésta. Los ingresos de las familias están determinados por $M=w_{C}^{0} F_{C}^{0}+\sum_{t=0}^{\infty}\left(w_{L}^{t} F_{L}^{t}+T_{g}^{t}+T_{e}^{t}\right)$.

Condiciones de ingresos balanceados

$$
\sum_{t=0}^{\infty}\left[(1+\tau) \sum_{i=1}^{5} p_{i}^{t} X_{i}^{t}+A_{p}^{t}\right]=w_{C}^{0} F_{C}^{0}+\sum_{t=0}^{\infty}\left(w_{L}^{t} F_{L}^{t}+T_{g}^{t}+T_{e}^{t}\right)
$$

\subsubsection{Condiciones de frontera para un modelo con horizonte infinito}

Se hace notar que las condiciones descritas previamente no incluyen el crecimiento del trabajo, del capital, y de la inversión a lo largo del horizonte de planeación, las que son importantes por su papel en la cerradura del modelo; dichas condiciones son denominadas de frontera y se deben satisfacer para asegurar que la economía se encuentra en una trayectoria estable a partir de cierto periodo $T$, a pesar de que modelo tiene un horizonte de planeación infinito, y de esta manera garantizar que las cantidades como el PIB, el capital, las inversiones, los consumos y las demandas no varían después del periodo $T$. 
Los modelos dinámicos, situados en el marco de la teoría del control óptimo, como se mencionó previamente se pueden abordar por medio de ecuaciones en diferencias para el caso discreto, cuya solución define el llamado retrato fase, que en el caso más sencillo define al consumo en función del capital acumulado como una curva cóncava hacia abajo. En este retrato fase es posible dividir, en el caso elemental, al primer cuadrante en regiones. En alguna de estas regiones es posible encontrar ciertas curvas que se conocen como trayectorias estables, se dice que la solución de un MEGC dinámico se encuentra en una trayectoria estable cuando para cada nivel del capital acumulado existe un único valor para el consumo de tal forma que la economía, como un sistema, tienda a un estado estacionario óptimo. La economía se encuentra en este estado cuando las variables macroeconómicas que determinan el trabajo, capital, consumo y producción crecen a una tasa constante $g$; en principio, la economía definida por la SAM se encuentra en un estado estacionario.

Resulta importante determinar las condiciones de crecimiento que deben satisfacer el capital y la inversión agregados para los periodos $t \geq T+1$, suponiendo que la solución debe encontrarse en un estado estacionario a partir del periodo $T$, es decir, se debe consolidar que las variables trabajo y capital crecen a una tasa constante $g$.

Por un lado, las condiciones de cero beneficio (Sec. 5.2.3) aseguran que para una inversión positiva $I^{t}$, se cumple que el precio del capital $P_{C}^{t+1}=p^{t}$, y debido a que la tasa de interés $r$ es aplicable a todos los precios, en particular para el precio de la producción agregada en el periodo $t-1$ se satisface:

$$
p^{t-1}=(1+r) p^{t}=P_{C}^{t}
$$

Por otro lado, una de las condiciones complementarias establece que el precio del capital en el periodo $t$ depende de la depreciación del precio de éste en el periodo $t+1$ más la renta del capital, $P_{C}^{t}=(1-\delta) P_{C}^{t+1}+w_{C}^{t}$, de donde el precio de la renta del capital es proporcional al precio $p^{t}$ de la producción agregada de la siguiente manera

$$
w_{C}^{t}=(r+\delta) p^{t}
$$

Si se supone que para $t \geq T+1$ la solución se encuentra en un estado estacionario, entonces la tasa de crecimiento del capital es $g$, por lo que se satisface la ecuación

$$
F_{C}^{t+1}=(1+g) F_{C}^{t}
$$

Se observa que la ecuación (5.4) no depende de la inversión, entonces al ser sustituida en la ecuación (5.1) que define el crecimiento del capital en el periodo $t+1$ se obtiene la igualdad

$$
F_{C}^{t+1}=(1+g) F_{C}^{t}=(1-\delta) F_{C}^{t}+I^{t}
$$


para $t \geq T+1$

Entonces es posible caracterizar a la inversión agregada, en el estado estacionario, como:

$$
I^{t}=(g+\delta) F_{C}^{t}
$$

para $t \geq T+1$.

La ausencia de inversión en el crecimiento del capital se refleja en que la renta del capital $R C^{t}$ en el periodo $t$ satisface las igualdades $R C^{t}=w_{C}^{t} F_{C}^{t}=(r+\delta) p^{t} F_{C}^{t}$, de esta ecuación se despeja al capital.

$$
F_{C}^{t}=\frac{R C^{t}}{(r+\delta) p^{t}}
$$

y se sustituye en (5.6), generando así a la inversión en función de la renta del capital a través de:

$$
I^{t}=\frac{(g+\delta) R C^{t}}{(r+\delta) p^{t}}
$$

La ecuación (5.7) en el periodo inicial $t=0$ está determinada por la ecuación

$$
I^{0}=\frac{(g+\delta) R C^{0}}{(r+\delta) p^{0}}
$$

y dado que en el periodo inicial el valor del capital coincide con el capital total de la SAM, y todos los precios se suponen unitarios; entonces la inversión inicial debe satisfacer la igualdad

$$
I^{0}=\frac{(g+\delta) F_{C}^{0}}{(r+\delta)}
$$

En conclusión, el capital inicial de la economía determina de manera recursiva el capital para cada periodo utilizando la ecuación (5.1), y además al satisfacer la ecuación (5.9) se obliga a los parámetros macroeconómicos $g, \delta$ y $r$ a satisfacer esta igualdad.

Finalmente, existe una condición que se utiliza para determinar la razón de crecimiento de la inversión en el periodo final $T$,

$$
\frac{I^{T}}{I^{T-1}}=\frac{Y^{T}}{Y^{T-1}}
$$

Esta condición afirma que las tasas de crecimiento de la inversión y de la producción son la misma, así la economía se obliga a que estas cantidades crezcan a la misma tasa a partir del último periodo de planeación sin requerir a priori que el modelo se encuentre en un estado estacionario de crecimiento. 
Este análisis se basa en que todas las cantidades crecen a la misma tasa en un estado estacionario. Así la inversión neta en el periodo terminal está determinada por el capital acumulado en el periodo terminal, la tasa de crecimiento exógena $g$ y la tasa de depreciación del capital $\delta$.

\subsection{Aproximación para un modelo Ramsey con ho- rizonte infinito}

Para obtener una aproximación a la solución se supone que el horizonte de planeación es separable, entonces se requiere descomponer la función objetivo del modelo del consumidor como la utilidad definida en los primeros periodos $t=0, \ldots, T$ más la correspondiente desde $t=T+1$ hasta infinito; a continuación se procede a desacoplar la restricción correspondiente de acuerdo a esta separación. Esto conduce a plantear dos problemas, uno con horizonte de planeación finito para $t=1, \ldots, T$, y otro desde $t=T+1$ a infinito. Ambos están ligados por el valor del llamado valor terminal de los recursos $A_{T}$, que en el caso de la economía representada, corresponde al valor del capital acumulado $F_{C}^{T+1}$ al principio del periodo $T+1$.

La construcción se basa al suponer que el valor del capital terminal $P C^{T+1} F_{C}^{T+1}$ a partir del intervalo de tiempo $T+1$ queda fijo para un cierto nivel de consumo $X_{T}^{*}, \mathrm{y}$ éste último se estabiliza a lo largo del horizonte infinito.

El modelo con horizonte infinito está determinado por:

$$
\operatorname{máx} U_{p}\left(X_{1}^{t}, \ldots, X_{5}^{t}\right)=\sum_{t=0}^{\infty}\left(\frac{1}{1+\delta}\right)^{t} \Pi_{i=1, i \neq 4}^{5}\left(X_{i}^{t}\right)^{\alpha_{i}}
$$

sujeto a

$$
\sum_{t=0}^{\infty}\left[(1+\tau) \sum_{i=1}^{5} p_{i}^{t} X_{i}^{t}+A_{p}^{t}\right]=w_{C}^{0} F_{C}^{0}+\sum_{t=0}^{\infty}\left(w_{L}^{t} F_{L}^{t}+T_{g}^{t}+T_{e}^{t}\right)
$$

Entonces, el modelo se reescribe de la siguiente manera, para separarlo

$$
\operatorname{máx} U_{p}\left(X_{1}^{t}, \ldots, X_{5}^{t}\right)=\sum_{t=0}^{T}\left(\frac{1}{1+\delta}\right)^{t} \Pi_{i=1, i \neq 4}^{5}\left(X_{i}^{t}\right)^{\alpha_{i}}+\sum_{t=T+1}^{\infty}\left(\frac{1}{1+\delta}\right)^{t} \Pi_{i=1, i \neq 4}^{5}\left(X_{i}^{t}\right)^{\alpha_{i}}
$$

sujeto a

$$
\sum_{t=0}^{T}\left[(1+\tau) \sum_{i=1}^{5} p_{i}^{t} X_{i}^{t}+A_{p}^{t}\right]=w_{C}^{0} F_{C}^{0}+\sum_{t=0}^{T}\left(w_{L}^{t} F_{L}^{t}+T_{g}^{t}\right)-P C^{T+1} F_{C}^{T+1}
$$




$$
\sum_{t=T+1}^{\infty}\left[(1+\tau) \sum_{i=1}^{5} p_{i}^{t} X_{i}^{t}+A_{p}^{t}\right]=P C^{T+1} F_{C}^{T+1}+\sum_{t=T+1}^{\infty}\left(w_{L}^{t} F_{L}^{t}+T_{g}^{t}\right)
$$

Cuando el valor del capital terminal $P C^{T+1} F_{C}^{T+1}$ está fijo, entonces el problema se puede resolver como dos problemas de optimización, uno de ellos con horizonte finito $T$. La solución del problema con horizonte finito determina el consumo en los periodos $t=0,1, \ldots, T$, y es prácticamente el mismo que el correspondiente al modelo con horizonte infinito. 


\section{6 Resultados para el modelo Dinámico}

En el Capítulo 4 se identificaron dos posibles políticas impositivas, para este capítulo una de éstas consiste en imponer impuestos simultáneos al uso de las energías EPG y FPPC, en la que se obtiene un cambio favorable en la demanda del uso de las energías renovables, que a su vez impacta la demanda de la energía eléctrica. La otra política consiste en asignar subsidios al uso de las energías renovables. Los resultados obtenidos para el caso estático propician que estas dos políticas se efectúen y comparen con una elasticidad variable a lo largo del horizonte de planeación. Para el primer periodo $(t=0)$, que en este modelo representa la economía de referencia, se supone un escenario rígido $(\sigma=0.5)$ y para cada periodo adicional la elasticidad se incrementa en 0.4 unidades, de tal manera que al periodo $t=25$ se alcanza un escenario flexible con $\sigma=10$. En este capítulo primero se analiza para cada periodo de tiempo $t=1, \ldots, 25$ y para cada productor $(j=1, \ldots, 5)$ la demanda de las energías intermedias utilizadas en la producción de la energía agregada $E_{j}^{t}$. La demanda de estas energías depende de la capacidad de sustitución, que hay en la economía, de las energías fósiles por las renovables. Posteriormente se cuantifica el impacto de esta sustitución a nivel macroeconómico utilizando las variables agregadas que definen el consumo, la inversión, el PIB y la balanza comercial (exportaciones vs importaciones), para determinar el impacto de estas políticas en el crecimiento de la economía mexicana.

En la Secc. 6.1 se hace la calibración de los parámetros definidos por la economía mexicana y de aquellos definidos por las preferencias y tecnología de producción. En la Sección 6.2 se interpreta la variación de la demanda de los bienes intermedios al suponer que la tasa de impuestos o subsidios es $20 \%$, con el fin de cuantificar la sustitución de las energías de origen fósil por las renovable; asimismo se compara esta variación entre las dos políticas para medir el beneficio obtenido entre ellas. Uno de los objetivos primordiales de los modelos dinámicos es evaluar los impactos en el crecimiento 
económico al realizar cambios en las políticas económicas, los resultados correspondientes se presentan en la Secc. 6.3 bajo el supuesto de que las tasas utilizadas en las políticas impositivas son $20 \%, 40 \%$ y $60 \%$.

\subsection{Calibración y Simulaciones}

En esta sección se describen los procesos de calibración y simulación, bajo el supuesto de que se satisfacen las condiciones de equilibrio, descritas en el Capítulo 4, para cada uno de los agentes de la economía, así como las condiciones macroeconómicas.

La calibración de los modelos dinámicos requiere asignar el valor definido por la economía a dos de los tres parámetros siguientes: la tasa del crecimiento del trabajo $g$, la tasa de depreciación o descuento $\delta$ la renta del capital $r$. Estos tres parámetros deben satisfacer la ecuación (5.9), en este trabajo se fijan los valores de la tasa de descuento $\delta=\delta^{*}=0.01119$ y la renta del capital $r=r^{*}=0.075$, estableciendo así que la tasa de crecimiento de factor trabajo es $g=g^{*}=0.02$. El resto de los parámetros: fiscales, coeficientes de proporcionalidad y coeficientes de escala se mantienen en los valores obtenidos en la Sec. 4.1 debido a que las preferencias del consumidor y la tecnología de producción correspondientes son las mismas.

El análisis supone que la economía se encuentra distorsionada inicialmente por los impuestos y subsidios contenidos en el equilibrio de referencia y por tanto los parámetros de calibración se calculan bajo este supuesto. Como en el Capítulo 4 los procesos de calibración, validación y obtención de resultados se realizan por medio del modelador GAMS. 


\subsection{Análisis y comparativo de la variación de las demandas intermedias}

La Figura 6.1 muestra claramente que gravar el uso de las energías fósiles ocasiona un incremento cercano al $70 \%$ en la demanda del bien GEE, cuando la elasticidad es cercana a diez. Para esta misma elasticidad, a diferencia de la política impositiva los subsidios al uso de la energía renovable no ocasionan un incremento representativo en la demanda de este bien, al mantenerse a lo largo de los 25 años por debajo del $7 \%$.

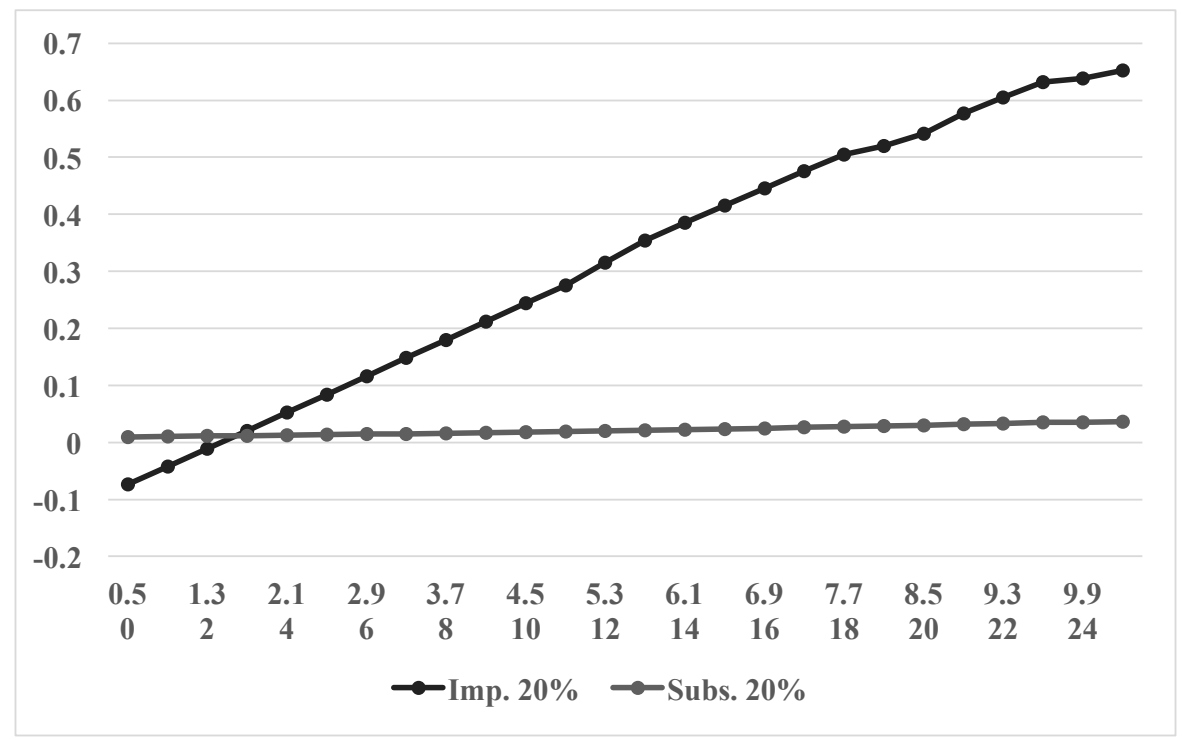

Figura 6.1: Porcentaje de variación de la demanda intermedia del bien GEE

Fuente: Elaboración propia. 
La gráfica que representa la demanda del bien fósil EPG indica que ésta decrece tanto bajo el gravamen, Fig. (6.2), como al asignar subsidios al uso de las energías renovables, en ambos casos a mayor elasticidad parece que la demanda se estabiliza por debajo del $40 \%$, originando que la diferencia de la demanda que existe entre ambas políticas sea menor.

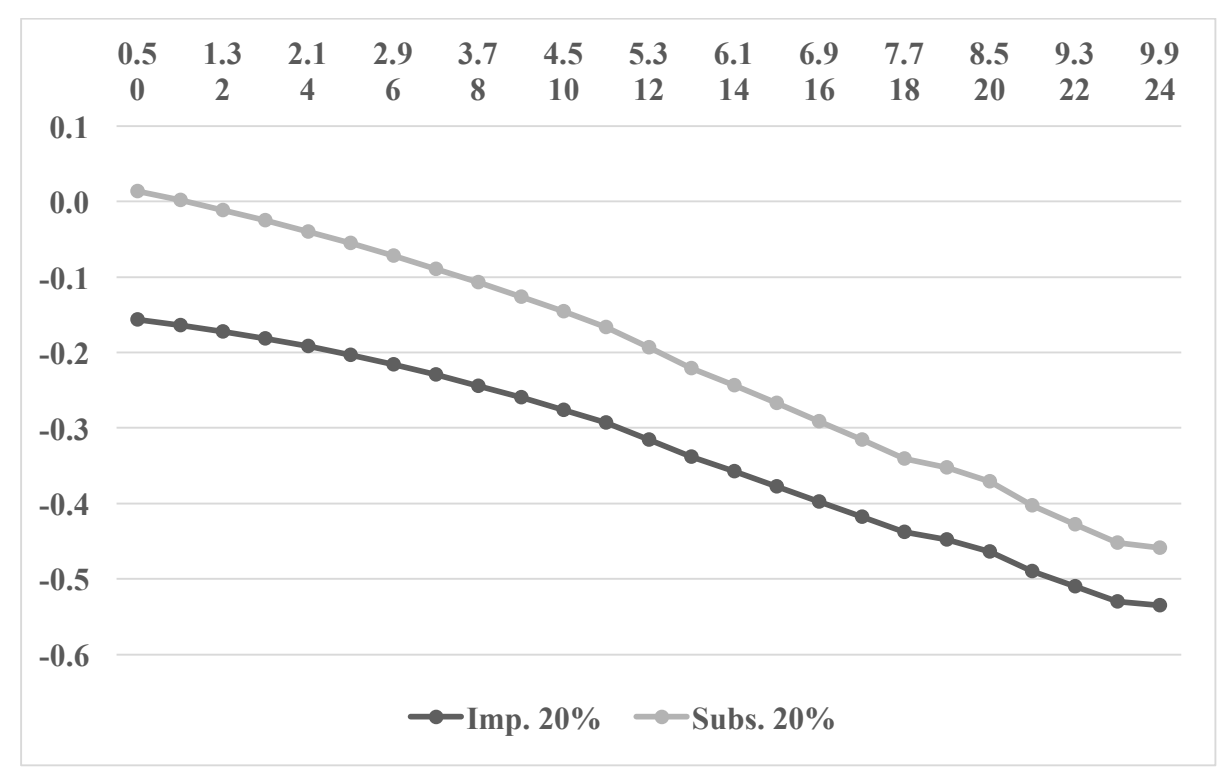

Figura 6.2: Porcentaje de variación de la demanda intermedia del bien EPG

Fuente: Elaboración propia. 
La demanda del bien fósil FPPC se decrementa en mayor porcentaje bajo impuestos que bajo subsidios como lo muestra la Fig. 6.3, lo que indica que el subsidio al uso de las energías renovables y la elasticidad de sustitución variable no impactan significativamente la demanda de este bien, ya que se mantiene estable y cercana a cero los primeros 6 o 7 años, con una elasticidad entre 2.9 y 3.3, para posteriormente decrecer hasta aproximadamente $10 \%$ al final del horizonte. Cuando se impone un gravamen al uso de las energías fósiles la demanda correspondiente decrece a mayor rapidez que bajo subsidios, hasta alcanzar aproximadamente $60 \%$.

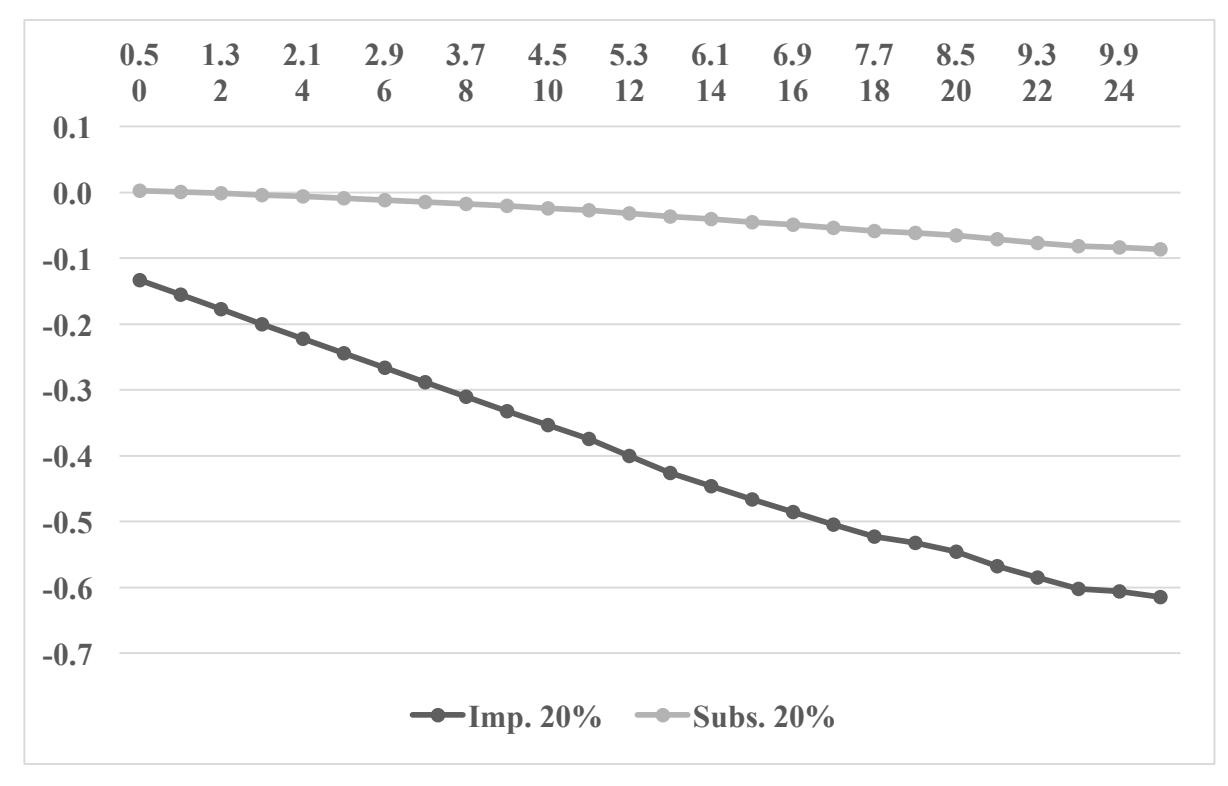

Figura 6.3: Porcentaje de variación de la demanda intermedia del bien FPPC

Fuente: Elaboración propia. 
Finalmente, la Fig. 6.4 hace evidente que el impacto a la demanda intermedia del bien REN bajo impuestos se incrementa por debajo del $200 \%$, pero el incremento cuando se asigna un subsidio es cercano al $400 \%$. A pesar de este impacto tan significativo, en lo que respecta a la demanda de este bien, no es claro que la asignación de subsidios sea la mejor política que deba seguir un decisor.

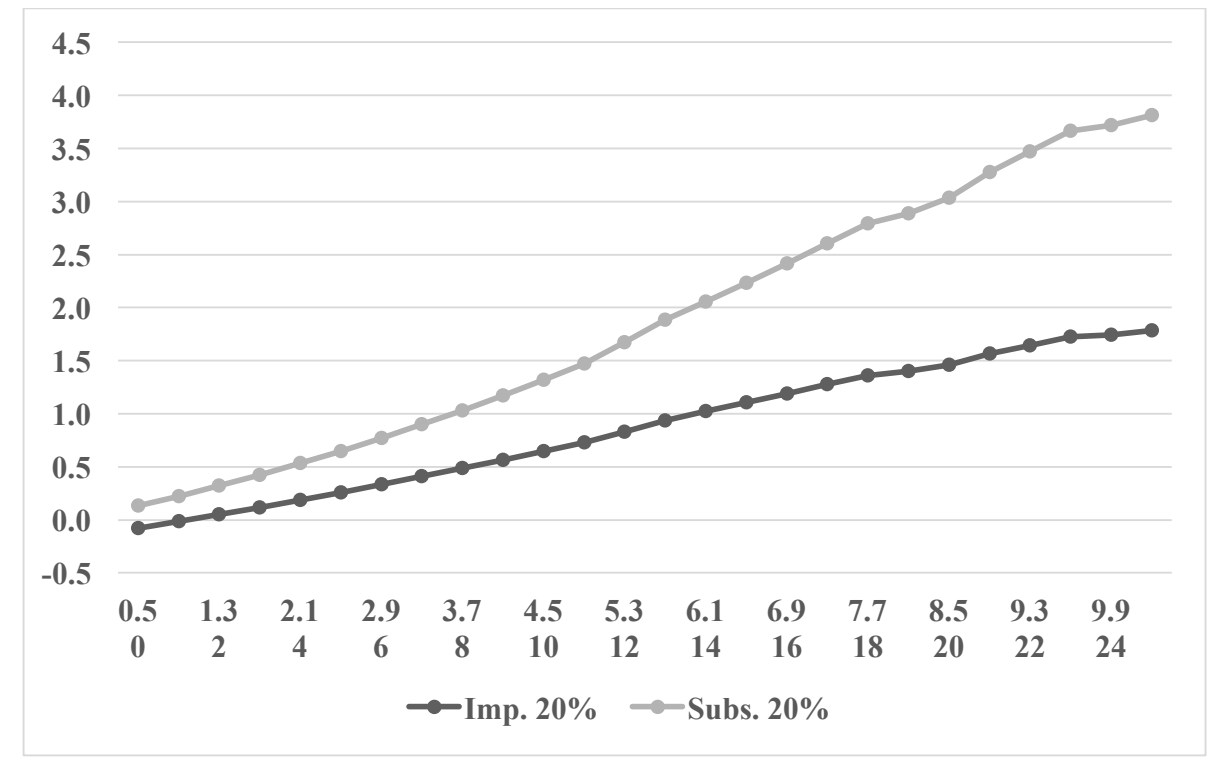

Figura 6.4: Porcentaje de variación de la demanda intermedia del bien REN

Fuente: Elaboración propia.

\subsection{Análisis del impacto macroeconómico}

El análisis de los datos obtenidos se realiza para cada política impositiva (impuestos o subsidios), para cada uno de los porcentajes siguientes $20 \%, 40 \%$ y $60 \%$, y se lleva a cabo en un horizonte de planeación de 25 años en el que varía la elasticidad de sustitución. El diagnóstico toma en cuenta el año cero como referencia para comparar y decidir.

En el caso de la política impositiva, se muestra que la tasa del $20 \%$ tiene menor impacto negativo sobre el comportamiento de las variables agregadas concernientes al consumo, inversiones, PIB y balanza comercial que definen el crecimiento económico. En contraste, para esa misma tasa en la asignación de subsidios, la pauta que muestran estas variables es de un claro crecimiento; razón que sustenta la comparación de las demandas intermedias de los bienes energéticos bajo esta tasa, Secc. 6.2. 


\subsubsection{Impactos al gravar el uso de energías fósiles}

El porcentaje de variación del consumo está por debajo del al escenario base, de acuerdo a la Fig. 6.5, para cualquiera de las diferentes tasas impositivas y se mantiene a una tasa casi constante. Por ejemplo, cuando el impuesto es $60 \%$ el consumo se mantiene $7 \%$ por debajo del correspondiente al equilibrio de referencia. Lo que significa que, aunque disminuya la demanda de las energías intermedias de origen fósil, los impuestos impactan negativamente este indicador macroeconómico.

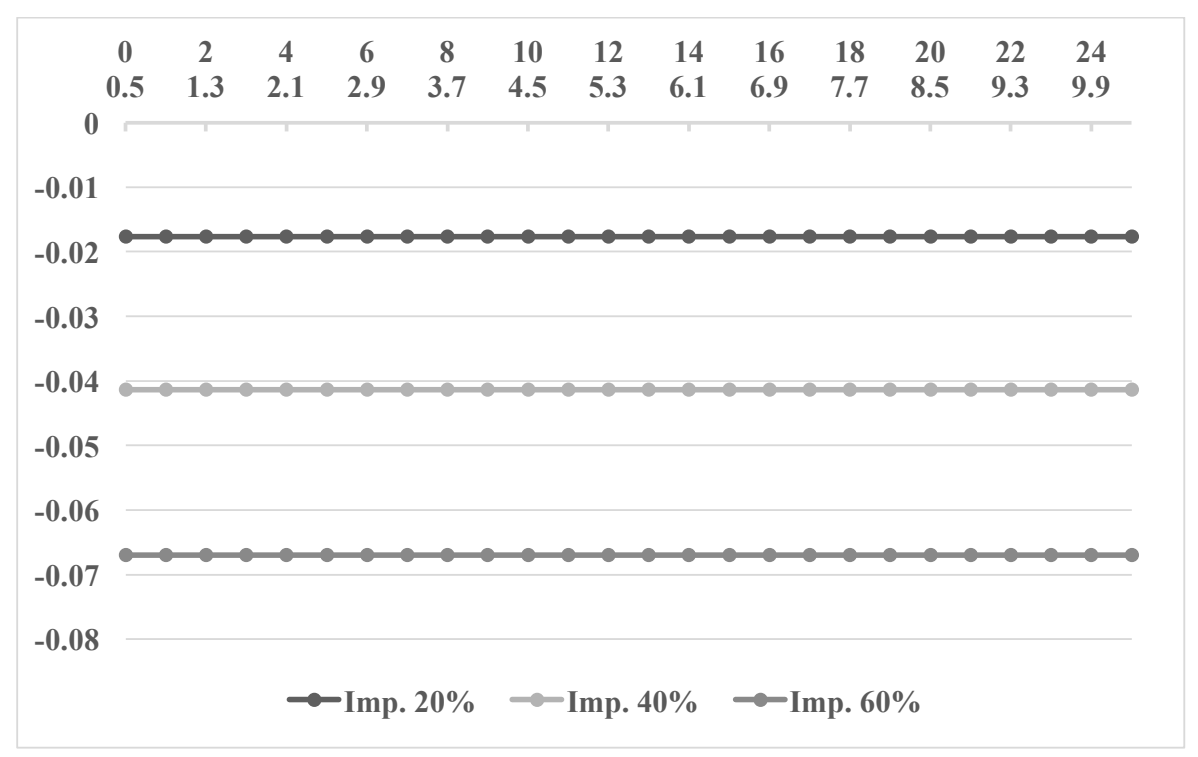

Figura 6.5: Porcentaje de variación del consumo agregado (Imp.)

Fuente: Elaboración propia. 
Es claro que la Fig. 6.6 señala que el impacto que afecta en menor proporción a la inversión agregada es el menor de los impuestos, $20 \%$, debido a que su valor inicial está por encima del relativo a los subsidios del $40 \%$ y $60 \%$, y a pesar de que en algunos periodos está ligeramente por debajo vuelve a dominar hasta el final del horizonte de planeación. En general, el mayor problema es que su crecimiento se mantiene por debajo del escenario de referencia.

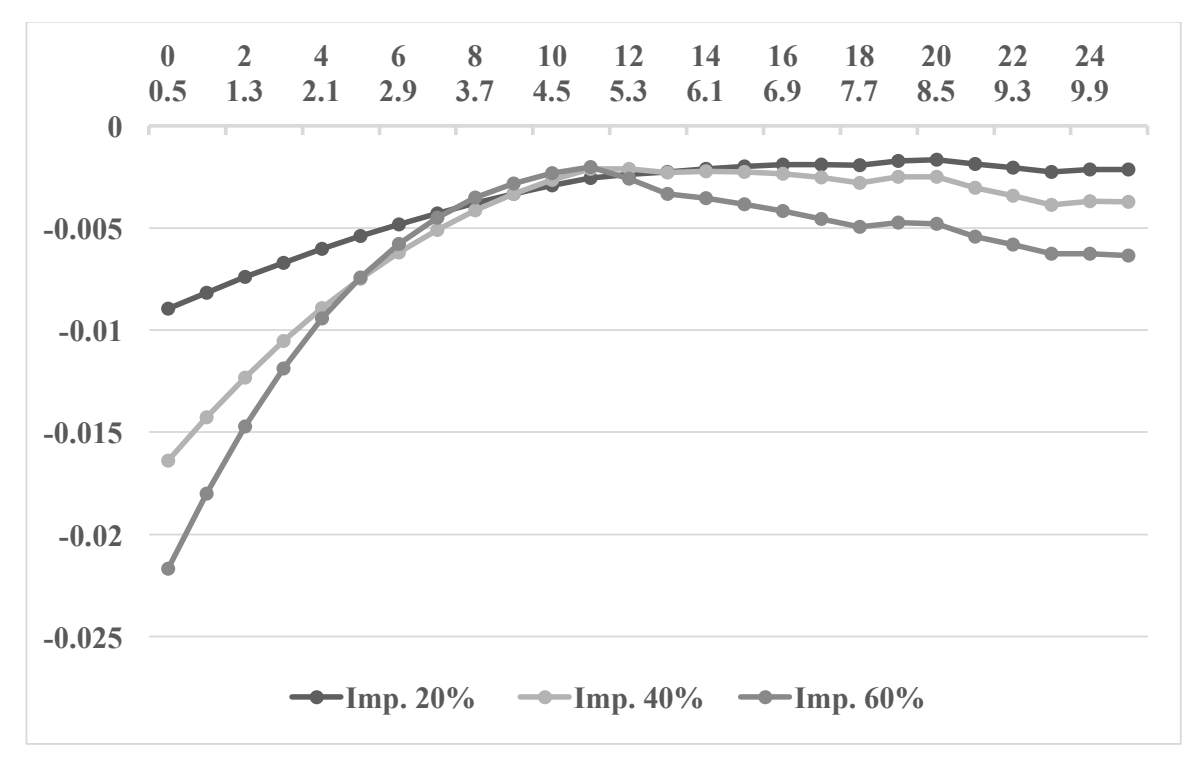

Figura 6.6: Porcentaje de variación de la inversión agregada (Imp.)

Fuente: Elaboración propia. 
La Figura 6.7 muestra que el PIB inicia y termina en un porcentaje por debajo del equilibrio de referencia, que tiende a recuperarse al final del horizonte de planeación debido a la elasticidad de sustitución. Se observa que este comportamiento inicial es incluso más significativo al incrementar los impuestos al uso de energías fósiles; esto se debe al comportamiento de la inversión y el consumo como se muestra en las Figuras 6.6 y 6.5 , respectivamente. Por lo tanto, aunque los impuestos disminuyen la demanda de los bienes energéticos de origen fósil EPG y FPPC esta reducción puede no ser suficiente para sustentar el uso de impuestos, ya que el impacto del consumo agregado al PIB se refleja en un porcentaje de decaimiento del PIB al inicio del horizonte y una ligera recuperación al final del horizonte, en el que la elasticidad es cercana a 10. El gravamen que afecta en menor proporción el comportamiento del PIB es $20 \%$.

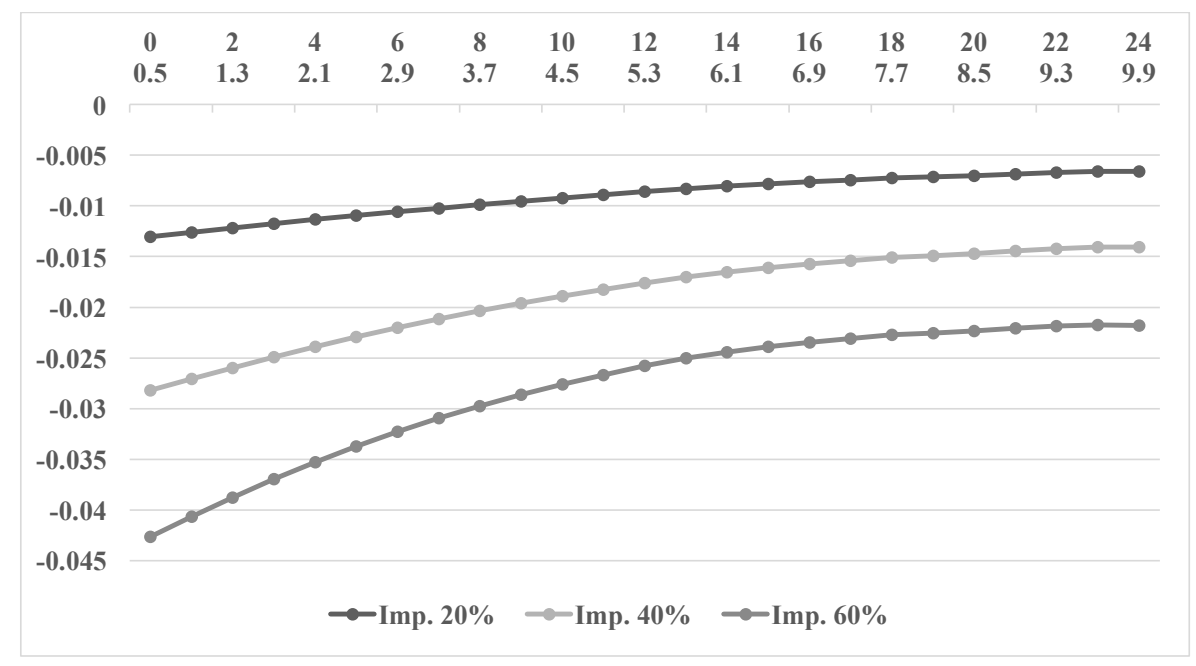

Figura 6.7: Porcentaje de variación del PIB (Imp.)

Fuente: Elaboración propia. 
El comportamiento de la balanza comercial se mide al evaluar y analizar cualitativamente la diferencia, en porcentaje, entre exportaciones e importaciones. Las curvas de la Fig. 6.8 muestran que para cada una de las tasas impositivas las exportaciones son menores que las importaciones; estos resultados no son alentadores, el hecho de que sean negativas aclara que tanto las exportaciones como las importaciones inician por debajo de su comportamiento en la economía de referencia. En general, las curvas presentan un comportamiento estable al final del horizonte; en particular, para una tasa impositiva del $20 \%$ la balanza comercial decae alrededor del $40 \%$ aproximadamente a los 22 años cuando la elasticidad tiene un valor de 9.3, este fenómeno se presenta con antelación (cercano a los 14 años para $\sigma=6.1$ ) para las tasas del $40 \%$ y $60 \%$ y el decrecimiento tiende a ser $60 \%$.

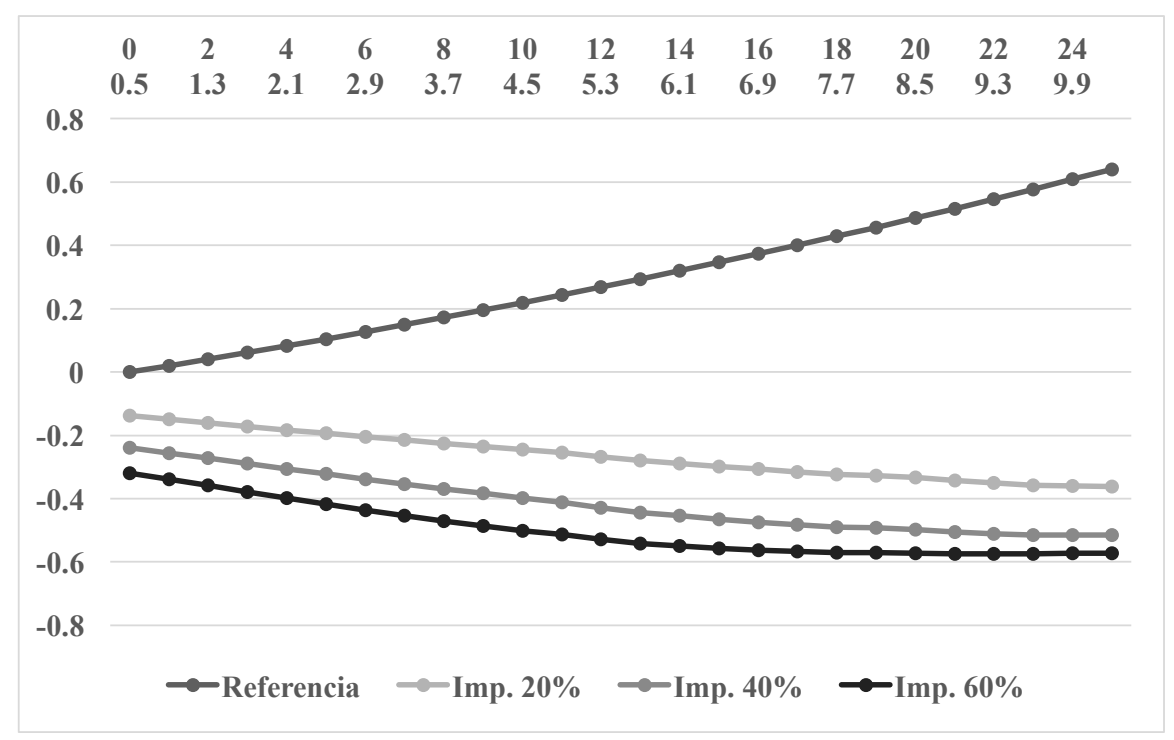

Figura 6.8: Balanza comercial en porcentaje (Imp.)

Fuente: Elaboración propia. 


\subsubsection{Impactos al asignar subsidios al uso de energías renova- bles}

El comportamiento del porcentaje de la variación del consumo agregado (ver Fig. 6.9) está por encima del escenario de referencia e indica que su crecimiento depende de la tasa del subsidio, pero no de la variación de la elasticidad. Esto quiere decir que el porcentaje de crecimiento del consumo agregado es casi constante a lo largo del horizonte de planeación, para cada una de las tasas de subsidio asignadas al uso de las energías renovables. La tasa de crecimiento más significativa apenas alcanza el $7 \%$ para un subsidio de $60 \%$, para el $20 \%$ no llega a ser el $1 \%$.

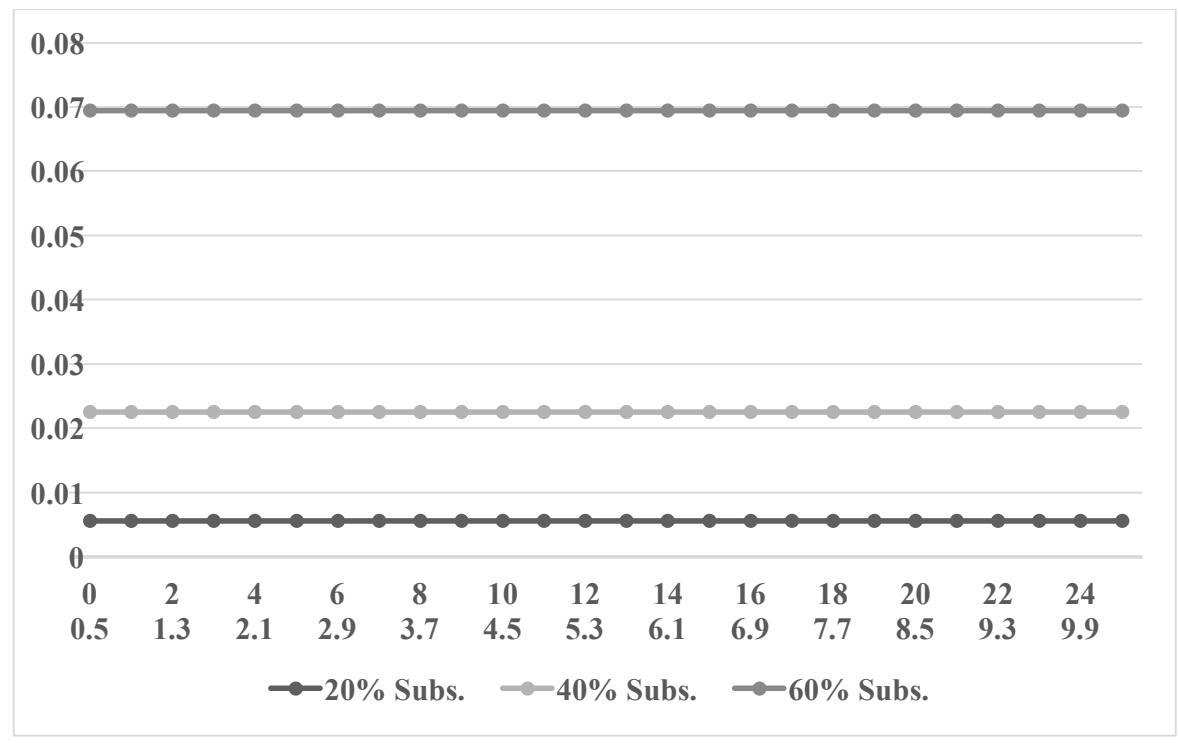

Figura 6.9: Porcentaje de variación del consumo agregado, (Sub.)

Fuente: Elaboración propia. 
La Fig 6.10 muestra que la variación del porcentaje para la inversión agregada se encuentra por encima de su valor en la economía de referencia y depende del valor de la tasa asignada al subsidio. Para las tasas del $20 \%$ y $40 \%$ el crecimiento de la inversión agregada es casi constante a lo largo del horizonte de planeación, lo que parece indicar que éste no depende de la elasticidad. En contraste, para una tasa del $60 \%$ durante los primeros 10 años la variación es una curva cóncava hacia abajo, alcanzando un valor máximo alrededor de los cuatro años cuando $\sigma=2.1$; después de los 10 años la curva decrece por debajo del valor inicial $(t=0)$ estabilizándose en un valor cercano a este valor inicial. En los tres casos a partir de algún periodo de tiempo se alcanza cierta estabilidad.

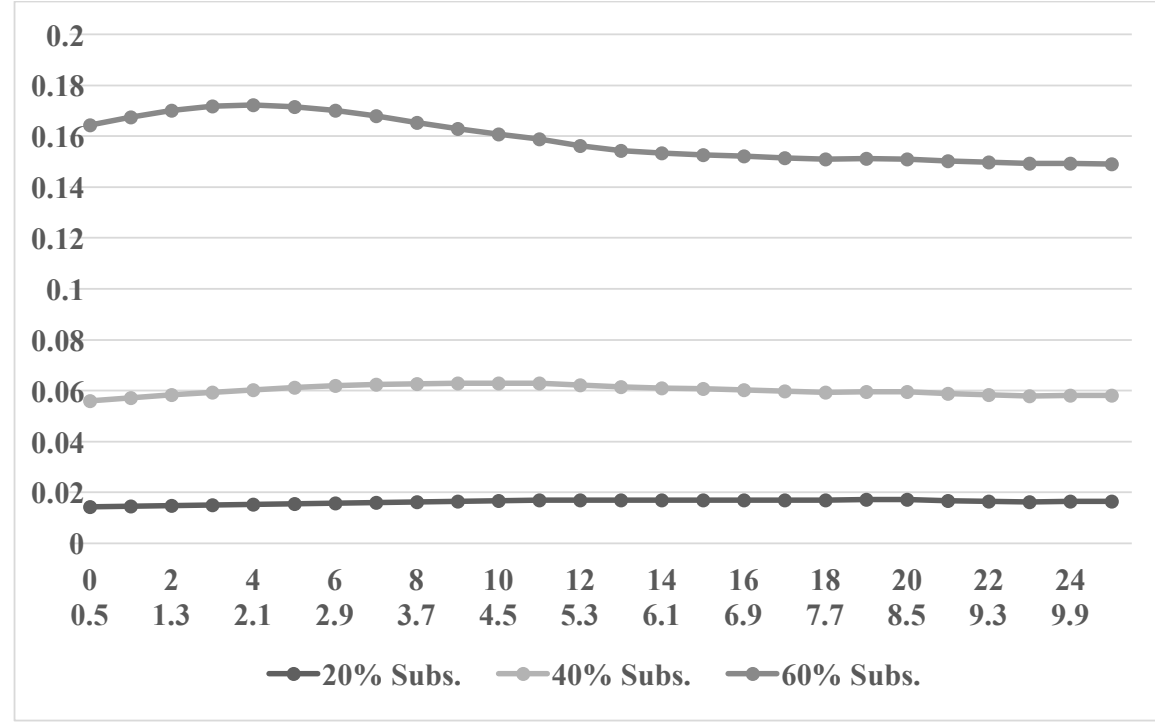

Figura 6.10: Porcentaje de variación de la inversión (Sub.)

Fuente: Elaboración propia. 
Para las tres tasas de asignación de subsidios al uso de energías renovables la Fig. 6.11 indica un incremento del PIB a una tasa casi constante que depende del subsidio, a mayor subsidio mayor es la tasa de crecimiento. Este comportamiento es semejante al del consumo agregado, lo que indica que los subsidios determinan el crecimiento del PIB, pero no así el cambio de tecnología. Esto se debe principalmente a que, el único consumidor del bien intermedio REN es el productor de energía eléctrica. Cabe destacar que para el máximo subsidio el PIB se mantiene alrededor del $10 \%$ por encima del correspondiente al equilibrio de referencia.

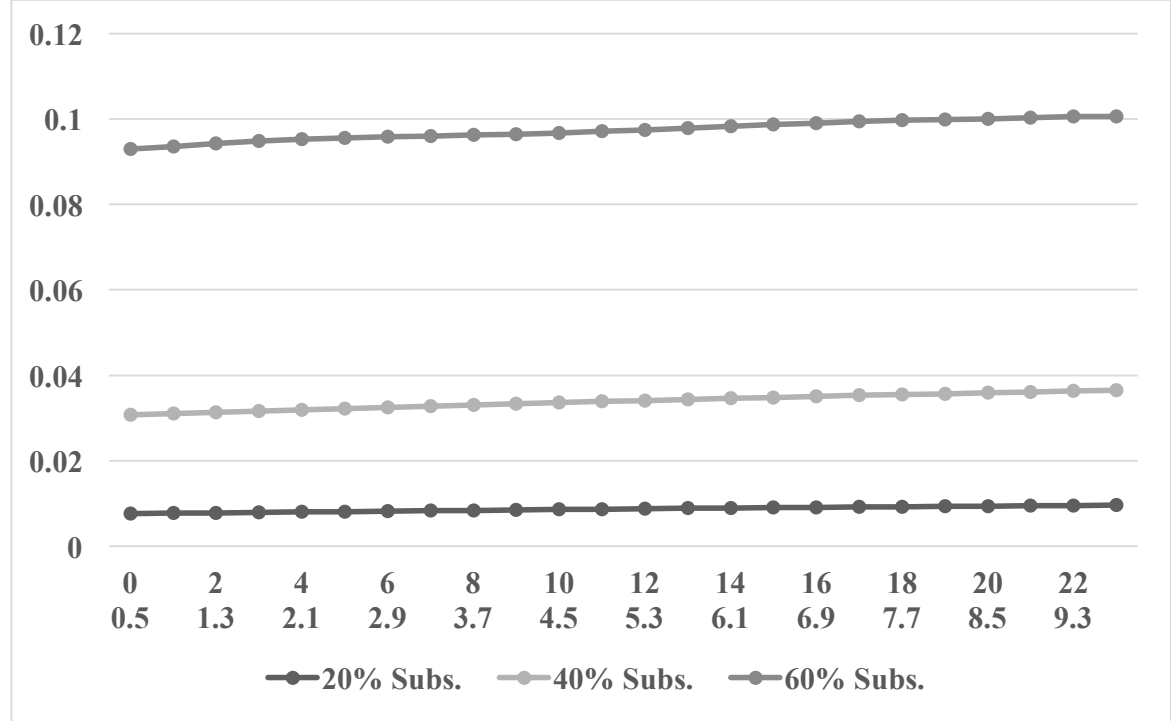

Figura 6.11: Porcentaje de variación del PIB (Sub.)

Fuente: Elaboración propia.

Para esta política, a diferencia de la anterior, la asignación de subsidios tiene un impacto menos nocivo en el comercio internacional. En particular, si el subsidio es $20 \%$ entonces la tasa de variación de la balanza comercial se mantiene cercana a cero, como se aprecia en la Fig. 6.12; el comportamiento cualitativo de la curva correspondiente revela que la balanza tiende a decrecer después de 10 años pero al final del horizonte de planeación desciende en menos del 1\%, lo que significa que las exportaciones e importaciones son casi iguales.

Para las tasas de $40 \%$ y $60 \%$, las curvas son positivas, decreciente y cóncavas hacia abajo mostrando inicialmente que la balanza comercial se encuentra por arriba del escenario base, pero alrededor de los ocho años se mantiene por debajo de éste. Este comportamiento se justifica al existir un incremento en las exportaciones pero 
alrededor de los 7 años, cuando la elasticidad de sustitución es 3.3, la diferencia entre exportaciones e importaciones es negativa y además tiende a cambiar de concavidad y decrecer aproximadamente hasta un $40 \%$ para un subsidio del $60 \%$; lo que implica que las exportaciones decrecen a mayor tasa que las importaciones en relación a la economía de referencia. En comparación al comportamiento bajo una tasa impositiva, resulta menos dañino optar por una política de subsidios al uso de energías renovables.

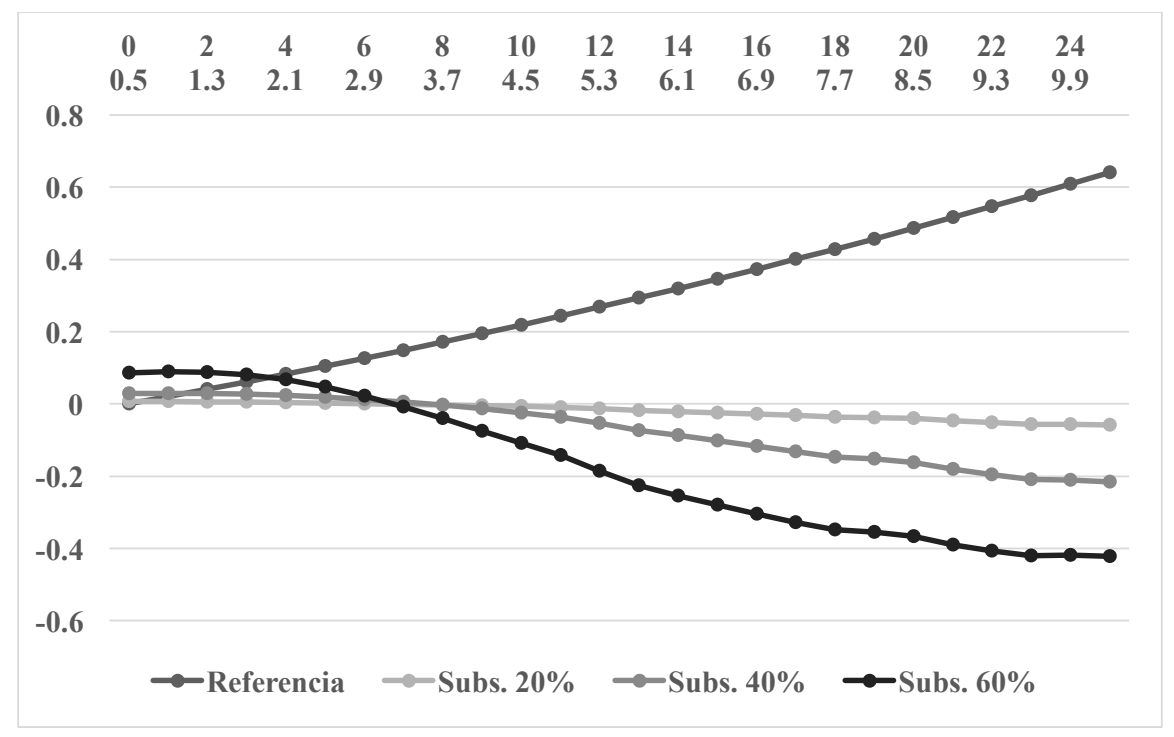

Figura 6.12: Balanza comercial en porcentaje (Sub.)

Fuente: Elaboración propia.

Para finalizar, en términos de la balanza comercial resulta menos pernicioso optar por una asignación de subsidios al uso de energías renovables que por un gravamen al uso de energías fósiles.

Los detalles de la variación porcentual en la balanza comercial, están contenidos en los Cuadros A.11 y A.12, en el que se presentan de manera simultánea los datos de ambas políticas fiscales, por año y valor de la elasticidad de sustitución. 


\section{7 \\ Conclusiones y Perspectivas}

En este trabajo se planteó la problemática de sustituir energías fósiles por renovables en el contexto de la economía sustentable, el objetivo se alcanzó al utilizar dos MEGC en los cuales se utilizaron dos políticas fiscales en diferentes escenarios relacionados con la elasticidad de sustitución.

En un inicio la problemática se aborda en forma estática, para definir las políticas fiscales y el escenario que promueven de manera eficiente, desde el punto de vista económico, la sustitución de energías. La solución al modelo estático determina el nivel de las demandas intermedias y de consumo final de los bienes energéticos, en dos escenarios. Se demuestra que la sustitución de las energías fósiles por el uso de energía renovables se realiza de manera eficiente cuando existe una mayor elasticidad de sustitución técnica; debido a esta conclusión resulta esencial estimar en un futuro el valor de este parámetro para la economía mexicana.

El modelo dinámico se desarrolla con un horizonte de planeación infinito que se estabiliza a los 25 años con una elasticidad variable respecto al tiempo, la que se incrementa a lo largo del horizonte de planeación desde un valor 0.5 , considerado como un escenario rígido, hasta un escenario flexible en el que la elasticidad es 10. El estudio para el modelo dinámico inicia con la calibración de los parámetros que intervienen en el crecimiento de las variables agregadas definidas por el trabajo, el capital y la inversión así como en la variación del precio de la producción agregada. La calibración de estos parámetros resulta ser compleja, ya que sus valores no se pueden fijar de manera única y deben reproducir el equilibrio de referencia. Se concluye que la asignación de subsidios es una política que permite el crecimiento del PIB mientras que el consumo agregado crece a una tasa constante.

Los modelos utilizan una matriz de contabilidad social, que representa la economía 
nacional y su adecuación requirió previamente de dos procesos: agregar la información en seis sectores representados por empresas y la estimación e incorporación de la información agregada de la demanda de las energías renovables como un bien intermedio. En este sentido resulta imperativo construir y actualizar la matriz de contabilidad social de la economía mexicana, con la debida incorporación de información que refleje las demandas intermedias de energéticos de origen fósil y renovable para cada sector, y de la demanda final de estas energías. Adicionalmente, es deseable tener la información desagregada por región, ya que las condiciones climatológicas varían en el país y por ende las demandas de energía.

Según el reporte de la OCDE del 2013, [24], México tiene uno de los porcentajes más bajos de participación del sector privado en el gasto bruto en investigación y desarrollo. En general, el marco para promover la innovación no ha sido eficaz y México se ha quedado lejos de alcanzar sus objetivos en cuanto al incremento de la productividad y la competitividad de la economía por medio de la innovación tecnológica, debido a la preferencia por la tecnología importada.

Por último, con el propósito de favorecer un mayor uso de las energías renovables en México, es indispensable invertir en la investigación que promueva el desarrollo y cambio tecnológico. En este sentido, Popp et al. [30] señalan que el costo de control y disminución de los problemas ambientales sería muy alto tomando en cuenta de forma exclusiva el uso de las tecnologías existentes; lo que hace necesaria la comprensión de la migración a nuevas tecnologías como parte importante de la proyección de los impactos al medio ambiente en un futuro. 


\section{Bibliografía}

[1] Aguayo, E., Chapa J. C., Ramírez N. C. y Rangel E., Análisis de la Generación y Redistribución del Ingreso en México a través de una Matriz de Contabilidad Social, en Estudios Económicos, Número extraordinario, 2011.

[2] Acemoglu, D., P. Aghion, L. Bursztyn, y D. Hemous, The Environment and Directed Technical Change, American Economic Review, Vol. 102 (1), 1, pp. 131-166, 2012.

[3] Acemoglu, D., P. Aghion, y D. Hemous, The Environment and Directed Technical Change in a North-South Model, Mimeo, 2013.

[4] Arrow, K. and G. Debreu, Existence of an Equilibrium for a Competitive Economy, Econometrica, Vol. 22 (3), pp. 265-290, 1954.

[5] Banse, M., H. van Mejil, A. Tabeau y G. Woltjer, EU Biofuel Policies Affect Global Agricultural Markets, en European of Agricultural Economics, Vol. 35 (2), pp. 117$141,2008$.

[6] Hourcade, J., M. Jaccard, C. Bataille, C. y F. Ghersi, Hybrid Modeling: New Answers to Old Challenges, Introduction to the Special Issue of The Energy Journal. Energy Journal, Vol. 27 (The Energy Journal special issue Hybrid Modeling of Energy Environment Policies), pp. 1-12, 2006.

[7] Böhringer, Ch., The synthesis of bottom-up and top-down in energy policy modeling, en Energy Economics, Vol. 20, pp. 233-248, 1998.

[8] Böhringer Ch. y T. Rutherford, Integrated assessment of energy policies: Decomposing top-down and bottom-up, Elsevier: Journal of Economic Dynamics and Control, Vol. 33, pp. 1648-1661, 2009.

[9] Boyd, R., y M. Ibarrarán, Costs of compliance with the Kyoto Protocol: a developing country perspective, en Energy Economics, Vol. 24, pp. 21-39, 2002. 
[10] Bravo, H., Castro J., y Gutiérrez M., Efectos distributivos de la aplicación de un impuesto a la demanda de combustibles fósiles, economía mexicana nueva época, Vol. Cierre de Época (II), pp. 407-439, 2013.

[11] Dixon, P., S. Osborne y M. T. Rimmer, The Economy-wide Effects in the United States of Replacing Crude Petroleum with Biomass, en Energy and Environment, Vol. 8 (6), pp. 709-722, 2007.

[12] Ferris, M., S. Dirkse y A. Meeraus, Mathematical Problems with Equilibrium Constraints: Automatic Reformulation and Solution via Constrained Optimization, en Frontiers in Applied General Equilibrium Modeling, Edited by Timothy J. Kehoe, T. N. Srinivasan. John Whalley. Cambridge, 2005.

[13] Fullerton, D. y G. Heutel, The General Equilibrium Incidence of Environmental Taxes, en Journal of Public Economics, Vol. 91 (3), pp. 571-591, 2007.

[14] Ginsburg, V. y M. Keyzer, The Structure of Applied General Equilibrium Models, The MIT Press, 1997.

[15] Govinda, R. y M. S. Ram, General equilibrium analysis of economic and environmental effects of carbon tax in a developing country: case of Thailand, en Environmental Economics and Policy Studies, 5, pp. 179-211, 2002.

[16] Greenaway, D., S. J. Leybourne, G.V. Reed y J. Whalley, Applied General Equilibrium Modelling: Applications, Limits and Future Development, HMSO, 1993.

[17] Gurgel, A., S. J. Reilly y S. Paltsev, Potential Land Use Implications of a Global Biofuels Industry, Journal of Agricultural and Food Industrial Organization, Vol. 5, Article 9, Special Issue: Explorations in Biofuels Economics Policy and History, 2007.

[18] Harker, P. T. y J.-S. Pang, Finite-Dimensional Variational Inequality and NonLinear Complementary Problems: A survey of Theory, Algorithms and Applications, Mathematical Programming, Vol. 48, pp. 161-220, 1990.

[19] Heer, B. y A. Maussner, Dynamical General Equilibrium Modeling: Computational Methods and Applications, Springer Verlag, Segunda ed., 2009.

[20] Kretschmer, B. y S. Peterson, Integrating Bioenergy into Computable General Equilibrium Models - A Survey, en Kiel Institute for the World Economy, núm. 1473, 2008. 
[21] Lau, M., A. Pahlke y T. F. Rutherford, Approximating Infinite-Horizont Models in a Complementary Format: A primer in Dynamic General Equilibrium Analysis, en Journal of Economic Dynamics and Control, Vol 26, pp. 577-609, 2002.

[22] Mathiesen, L., Computational Experience in Solving Equilibrium Models by Sequence of Linear Complementary Problems, en Operations Research, Vol. 33 (6), pp. 1225-1250, 1985.

[23] Mowers, M. y Rausch , Distributional and Efficiency Impacts of Clean and Renewable Energy Standards for Electricity, en MIT Joint Program on the Science and Policy of Global Change, Report No. 225, 2012.

[24] OCDE, Evaluaciones de la OCDE sobre el desempeño ambiental: México, OECD Publishing, 2013.

[25] Palmer, K., R. Sweeney y M. Allaire , Modeling Policies to Promote Renewable and Low-Carbon Sources of Electricity, en RV Backgrounder, 2010.

[26] Paltsev, S., Moving from Static to Dynamical General Equilibrium Economic Models (Notes for beginner in MPSGE), Massachusetts Institute of Technology, 2004.

[27] Paltsev, J., J. Reilly, H. Jacoby, R. Eckaus, J. McFarland, M. Sarofim, M. Asadoorian y M. Babiker, The MIT Emissions Prediction and Policy Analysis (EPPA) Model: Version 4, en MIT Joint Program on the Science and Policy of Global Change, Report No. 125, 2005.

[28] Paul, A., K. Palmer K. y M. Woerman, Modeling a Clean Energy Standard for Electricity: Policy Design Implications for Emissions, Supply, Prices, and Regions, Energy Economics 36, pp. 108-124, 2013.

[29] Pearson, K., B. R. Parmenter, A. A. Powell, P. J. Wilcoxen y P. B. Dixon, P. Notes and Problems in Applied General Equilibrium Economics, Elsevier, Primera ed., 1992.

[30] Popp, D., R. G. Newell y A. B. Jaffe, Energy, the Environment, and Technological Change, en Handbook of the Economics of Innovation 2, ed. by B. Hall, and N. Rosenberg, chap. 21, pp. 873-937. Elsevier, Amsterdam, 2010.

[31] Rausch, S., G. E. Metcalf y J. M. Reilly, Distributional Impacts of Carbon Pricing: A General Equilibrium Approach with Micro-Data for Households, en Energy Economics, Vol. 33, pp. S20-S33, 2011. 
[32] Rausch, S. y T. F. Rutherford, Computational Equilibria in OLG Models with Many Heterogeneous Households, Computational Economics, Vol. 36 (2), pp. 171189, 2010.

[33] Romero, J., Energía, emisiones y precios relativos, en Yúnez-Naude, A. (Ed.), Medio Ambiente: Problemas y Soluciones, El Colegio de México, 1994.

[34] Rutherford, T., Extension of GAMS for complementary problems arising in applied economic analysis, en Journal of Economic Dynamics and Control, Vol. 19, pp. 1299-1324, 1995a.

[35] Rutherford, T., Applied General Equilibrium Modeling with MPSGE as GAMS Subsystem: An Overview of the Modeling Framework and Syntax, en Department of Economics, University of Colorado, 1995b.

[36] Rutherford, T., Dynamic General Equilibrium with GAMS/MPSGE, en Lectures Notes Prepared for the UNSW Workshop, 2004.

[37] Saam, M., C. Papageorgiou y P. Schulte, Elasticity of Substitution between Clean and Dirty Energy Inputs - A Macroeconomic Perspective, Discussion Paper No. 13-087, 2013.

[38] Sala i Martin, X., Apuntes de crecimiento económico, Antoni Bosch Editor S.A., 2000.

[39] Scarf, The approximation of fixed points of a continuous mapping, SIAM Journal of Applied Mathematics (15), pp. 1328-1342, 1967.

[40] Scarf, The computation of Economic Equilibria, Yale University Press, New Haven, 1973.

[41] SENER, Balance Nacional de Energía: México, Subsecretaría de Planeación y Transición Energética, Dirección General de Planeación e Información Energéticas, SENER, México, 2014, disponible en https://www.gob.mx/cms/uploads/attachment/file/44353/Balance_Nacional _de_Energ_a_2014.pdf

[42] Shoven, T., Applying General Equilibrium, Cambridge University Press, 1995. 


\section{A Anexo}

\section{A.1. Matriz de contabilidad social}

Cuadro A.1: Primera parte de la SAM, contiene a la submatriz de insumo-producto

\begin{tabular}{ccccccc} 
Actividad & SPR & ISE & GEE & EPG & FPP & REN \\
\hline SPR & 65937 & 391993 & 127 & 1982 & 934 & \\
ISE & 111204 & 4073079 & 77206 & 7570 & 14155 & \\
GEE & 7196 & 133118 & 29846 & 182 & 720 & \\
EPG & 0 & 934 & 45435 & 0 & 0 & \\
FPP & 2550 & 139878 & 674 & 0 & 0 & \\
REN & & & 11539 & & & \\
\hline LB & 53059 & 1780681 & 35300 & 11994 & 7045 & 347 \\
K & 233068 & 4231429 & 36259 & 57518 & 9272 & 8067 \\
\hline
\end{tabular}

FAM

$\begin{array}{llllll}\text { GOB } & 56812 & 806344 & -1578 & 69483 & 1942\end{array}$

$\mathrm{AHO}$

$\begin{array}{llllll}\text { SE } & 126139 & 2244061 & 27881 & 0 & 214142\end{array}$

$\begin{array}{lllllll}\text { TOT } & 655965 & 13801517 & 262689 & 148729 & 248210 & 11539\end{array}$ 
Cuadro A.2: Segunda parte de la SAM: ingresos de la familia por la renta de los factores y sus egresos

\begin{tabular}{cccc} 
Actividad & LB & K & FAM \\
\hline & & & \\
SPR & & & 122348 \\
ISE & & & 4972008 \\
GEE & & & 80369 \\
EPG & & & 0 \\
FPP & & & 78687 \\
REN & & & 0 \\
\hline LB & & & \\
K & & & \\
\hline & & & \\
FAM & 2166581 & 4622565 & \\
GOB & & & 368114 \\
AHO & & & 1564661 \\
SE & & 80483 & \\
\hline & & & \\
TOT & 2166581 & 4703048 & 12256757
\end{tabular}


Cuadro A.3: Tercera parte de la SAM, egresos del gobierno, de las inversiones y del sector externo

\begin{tabular}{cccc} 
Actividad & GOB & AHO & SE \\
\hline SPR & 2566 & 6239 & 63839 \\
ISE & 592323 & 1689683 & 2264289 \\
GEE & 9383 & 0 & 1875 \\
EPG & 0 & 124 & 102236 \\
FPP & 769 & 3200 & 22452 \\
REN & 0 & 0 & 0 \\
\hline LB & 275030 & & 0 \\
K & 127435 & & 0 \\
\hline FAM & 181031 & & 216010 \\
GOB & & & \\
AHO & 60247 & & 77071 \\
SE & 52333 & 2733 & 0 \\
\hline TOT & 1301117 & 1701979 & 2747772
\end{tabular}

En donde $\mathbf{L B}$ y $\mathbf{K}$ denotan los factores trabajo y capital respectivamente. 


\section{A.2. Desigualdades variacionales}

Formalmente la definición de un problema de desigualdades variacionales consiste en:

Definición A.1. Dado un subconjunto $X$ no vacío de $\mathbb{R}^{n}$ y una función $F$ tal que $F: \mathbb{R}^{n} \longrightarrow \mathbb{R}^{n}$. El problema de desigualdades variacionales consiste en determinar un vector $x^{*} \in X$ que satisface el conjunto de ecuaciones $y$ desigualdades definido por

$$
F\left(x^{*}\right)\left(y-x^{*}\right) \geq 0
$$

para toda $y \in X$.

En particular,

Definición A.2. Si $X$ un subconjunto convexo de $\mathbb{R}^{n}$, y $F(x)$ representa el gradiente de una función $f: \mathbb{R}^{n} \longrightarrow \mathbb{R}$, entonces el problema variacional es llamado complementario no lineal y el conjunto de ecuaciones y desigualdades variacionales es representado por las condiciones de primer orden del problema de optimización

$$
\min f(x)
$$

sujeto a que $x \in \mathbb{R}$

Para que $F(x)$ sea el gradiente de la función $f(x)$ se requiere que $F(x)$ sea continuamente diferenciable y que la matriz Jacobiana, $\nabla F(x)$, sea simétrica para toda $x \in \mathbb{R}^{n}$.

La función objetivo $f(x)$ se obtiene a partir de la integral

$$
f(x)=\int_{0}^{1} F\left(x_{0}+t\left(x-x_{0}\right)\right)^{T}\left(x-x_{0}\right) d t
$$

razón por la que se les conoce como problemas integrables.

Los problemas complementarios no lineales son un caso particular de las desigualdades variacionales, y se puede demostrar que algunos modelos de equilibrio general corresponden a este caso particular. Estos problemas consisten en determinar un vector $x^{*} \in \mathbb{R}_{+}^{n}$ tal que

$$
F\left(x^{*}\right) \in \mathbb{R}_{+}^{n}
$$

$\mathrm{y}$

$$
F\left(x^{*}\right)^{T} x^{*}=0
$$

Cabe señalar que un problema complementario no lineal difiere de un problema de optimización no lineal, el que se define como: 
Definición A.3. Dadas dos funciones $f: \mathbb{R}^{n} \longrightarrow \mathbb{R}$ y $g: \mathbb{R}^{n} \longrightarrow \mathbb{R}^{n}$, y sean $l=l^{*}$ y $u=u^{*}$ dos vectores en $\mathbb{R}^{n}$. Encontrar un vector $x^{*} \in \mathbb{R}_{+}^{n}$ que determina

$$
\max f(x)
$$

sujeto a $g(x)=0$

$l^{*} \leq x \leq u^{*}$

En el caso particular en que $F(x)$ es una transformación afín, $F(x)=q+M x$, para un vector $q \in \mathbb{R}^{n}$ y una matriz $M \in \mathbb{R}^{n \times n}$, el modelo es complementario lineal.

Existen otros casos particulares de interés, como el problema complementario generalizado definido como:

Definición A.4. Sea $X$ un cono convexo no vacío en $\mathbb{R}^{n}$ y sea $F$ una función de $F: \mathbb{R}^{n} \longrightarrow \mathbb{R}^{n}$. El objetivo consiste en determinar un vector $x^{*} \in X$ tal que se satisfacen las siguientes condiciones

$$
F\left(x^{*}\right) \in X^{*}
$$

$y$

$$
F\left(x^{*}\right)^{T} x^{*}=0
$$

donde $X^{*}=\left\{y \in R^{n}: y^{T} x \geq 0, x \in X\right\}$ denota el cono dual de $X$.

El primer resultado que fundamentó la relación entre el problema complementario generalizado y el problema de desigualdades variacional se debe a Karamardian [], y se enuncia como

Teorema A.1. Sea $X$ un cono convexo, entonces $x^{*} \in X$ resuelve el problema variacional si $y$ solo si $x^{*}$ resuelve el problema complementario generalizado. 


\section{A.3. Comparativo de la demanda de energías inter- medias}

Cuadro A.4: Demanda de energías intermedias para elasticidad variable

\section{Demanda de energías intermedias \\ (Impuestos vs Subsidios)}

\begin{tabular}{ccccc} 
& & \multicolumn{3}{c}{ GEE } \\
\cline { 3 - 5 } Añ & Elas. & $0 \%$ & Imp. & Subs. \\
\cline { 3 - 5 } $\mathbf{0}$ & $\mathbf{0 . 5}$ & 0 & -0.073 & 0.010 \\
$\mathbf{1}$ & $\mathbf{0 . 9}$ & 0.020 & -0.042 & 0.011 \\
$\mathbf{2}$ & $\mathbf{1 . 3}$ & 0.040 & -0.011 & 0.011 \\
$\mathbf{3}$ & $\mathbf{1 . 7}$ & 0.061 & 0.021 & 0.012 \\
$\mathbf{4}$ & $\mathbf{2 . 1}$ & 0.082 & 0.052 & 0.013 \\
$\mathbf{5}$ & $\mathbf{2 . 5}$ & 0.104 & 0.084 & 0.013 \\
$\mathbf{6}$ & $\mathbf{2 . 9}$ & 0.126 & 0.116 & 0.014 \\
$\mathbf{7}$ & $\mathbf{3 . 3}$ & 0.149 & 0.148 & 0.015 \\
$\mathbf{8}$ & $\mathbf{3 . 7}$ & 0.172 & 0.180 & 0.016 \\
$\mathbf{9}$ & $\mathbf{4 . 1}$ & 0.195 & 0.212 & 0.017 \\
$\mathbf{1 0}$ & $\mathbf{4 . 5}$ & 0.219 & 0.244 & 0.018 \\
$\mathbf{1 1}$ & $\mathbf{4 . 9}$ & 0.243 & 0.275 & 0.019 \\
$\mathbf{1 2}$ & $\mathbf{5 . 3}$ & 0.268 & 0.315 & 0.020 \\
$\mathbf{1 3}$ & $\mathbf{5 . 7}$ & 0.294 & 0.354 & 0.021 \\
$\mathbf{1 4}$ & $\mathbf{6 . 1}$ & 0.319 & 0.385 & 0.022 \\
$\mathbf{1 5}$ & $\mathbf{6 . 5}$ & 0.346 & 0.415 & 0.024 \\
$\mathbf{1 6}$ & $\mathbf{6 . 9}$ & 0.373 & 0.446 & 0.025 \\
$\mathbf{1 7}$ & $\mathbf{7 . 3}$ & 0.400 & 0.476 & 0.026 \\
$\mathbf{1 8}$ & $\mathbf{7 . 7}$ & 0.428 & 0.505 & 0.028 \\
$\mathbf{1 9}$ & $\mathbf{8 . 1}$ & 0.457 & 0.520 & 0.028 \\
$\mathbf{2 0}$ & $\mathbf{8 . 5}$ & 0.486 & 0.542 & 0.030 \\
$\mathbf{2 1}$ & $\mathbf{8 . 9}$ & 0.516 & 0.577 & 0.032 \\
$\mathbf{2 2}$ & $\mathbf{9 . 3}$ & 0.546 & 0.605 & 0.033 \\
$\mathbf{2 3}$ & $\mathbf{9 . 7}$ & 0.577 & 0.632 & 0.035 \\
$\mathbf{2 4}$ & $\mathbf{9 . 9}$ & 0.608 & 0.639 & 0.036 \\
$\mathbf{2 5}$ & $\mathbf{1 0}$ & 0.641 & 0.652 & 0.037 \\
& & & &
\end{tabular}

\begin{tabular}{cc}
\multicolumn{2}{c}{ EPG } \\
\hline Imp. & Subs. \\
\hline-0.156 & 0.014 \\
-0.164 & 0.002 \\
-0.172 & -0.011 \\
-0.181 & -0.025 \\
-0.192 & -0.040 \\
-0.203 & -0.055 \\
-0.216 & -0.072 \\
-0.230 & -0.089 \\
-0.244 & -0.107 \\
-0.259 & -0.126 \\
-0.276 & -0.146 \\
-0.293 & -0.166 \\
-0.315 & -0.193 \\
-0.339 & -0.221 \\
-0.358 & -0.244 \\
-0.377 & -0.267 \\
-0.397 & -0.291 \\
-0.418 & -0.316 \\
-0.438 & -0.340 \\
-0.448 & -0.353 \\
-0.464 & -0.371 \\
-0.489 & -0.403 \\
-0.510 & -0.428 \\
-0.530 & -0.452 \\
-0.535 & -0.458 \\
-0.545 & -0.471 \\
&
\end{tabular}

\begin{tabular}{cc}
\multicolumn{2}{c}{ FPPC } \\
\hline Imp. & Sub. \\
\hline-0.133 & 0.003 \\
-0.155 & 0.001 \\
-0.178 & -0.002 \\
-0.200 & -0.004 \\
-0.222 & -0.006 \\
-0.245 & -0.009 \\
-0.267 & -0.011 \\
-0.289 & -0.014 \\
-0.311 & -0.017 \\
-0.332 & -0.020 \\
-0.354 & -0.024 \\
-0.375 & -0.027 \\
-0.401 & -0.032 \\
-0.426 & -0.037 \\
-0.446 & -0.041 \\
-0.466 & -0.045 \\
-0.486 & -0.049 \\
-0.505 & -0.054 \\
-0.523 & -0.059 \\
-0.533 & -0.061 \\
-0.546 & -0.065 \\
-0.568 & -0.071 \\
-0.585 & -0.076 \\
-0.602 & -0.082 \\
-0.607 & -0.083 \\
-0.615 & -0.086 \\
& \\
\hline
\end{tabular}

\begin{tabular}{cc}
\multicolumn{2}{c}{ REN } \\
\hline Imp. & Sub. \\
\hline-0.076 & 0.134 \\
-0.014 & 0.224 \\
0.050 & 0.321 \\
0.117 & 0.424 \\
0.186 & 0.532 \\
0.258 & 0.648 \\
0.332 & 0.769 \\
0.408 & 0.897 \\
0.485 & 1.032 \\
0.565 & 1.173 \\
0.646 & 1.321 \\
0.728 & 1.474 \\
0.831 & 1.675 \\
0.936 & 1.883 \\
1.021 & 2.057 \\
1.106 & 2.234 \\
1.190 & 2.416 \\
1.275 & 2.602 \\
1.358 & 2.791 \\
1.400 & 2.887 \\
1.463 & 3.032 \\
1.564 & 3.274 \\
1.644 & 3.470 \\
1.723 & 3.666 \\
1.743 & 3.716 \\
1.782 & 3.814 \\
&
\end{tabular}




\section{Cuadros del análisis macroeconómico para elastici- dad variable}

Cuadro A.5: Indicadores macroeconómicos, Impuestos (0-8 años)

Indicadores macroeconómicos

Impuestos y elasticidad variable

\begin{tabular}{|c|c|c|c|c|c|c|c|c|c|}
\hline Consumo & & & & & & & & & \\
\hline Elasticidad & 0.5 & 0.9 & 1.3 & 1.7 & 2.1 & 2.5 & 2.9 & 3.3 & 3.7 \\
\hline Año & $\mathbf{0}$ & 1 & 2 & 3 & 4 & 5 & 6 & 7 & 8 \\
\hline Imp. $20 \%$ & -0.0177 & -0.0177 & -0.0177 & -0.0177 & -0.0177 & -0.018 & -0.018 & -0.018 & -0.018 \\
\hline Imp. $40 \%$ & -0.0237 & -0.0237 & -0.0237 & -0.0237 & -0.0237 & -0.024 & -0.024 & -0.024 & -0.024 \\
\hline Imp. $60 \%$ & -0.0256 & -0.0256 & -0.0256 & -0.0256 & -0.0256 & -0.026 & -0.026 & -0.026 & -0.026 \\
\hline \multicolumn{10}{|l|}{ Inversión } \\
\hline Imp. $20 \%$ & -0.0090 & -0.0082 & -0.0074 & -0.0067 & -0.0060 & -0.0054 & -0.0048 & -0.0043 & -0.0038 \\
\hline Imp. $40 \%$ & -0.0074 & -0.0061 & -0.0049 & -0.0038 & -0.0029 & -0.0021 & -0.0014 & -0.0008 & -0.0004 \\
\hline Imp. $60 \%$ & -0.0053 & -0.0037 & -0.0024 & -0.0013 & -0.0005 & 0.0001 & 0.0004 & 0.0006 & 0.0006 \\
\hline \multicolumn{10}{|l|}{ PIB } \\
\hline Imp. $20 \%$ & -0.0130 & -0.0126 & -0.0122 & -0.0117 & -0.0113 & -0.0110 & -0.0106 & -0.0102 & -0.0099 \\
\hline Imp. $40 \%$ & -0.0152 & -0.0145 & -0.0138 & -0.0132 & -0.0126 & -0.0120 & -0.0115 & -0.0110 & -0.0105 \\
\hline Imp. $60 \%$ & -0.0144 & -0.0136 & -0.0128 & -0.0121 & -0.0114 & -0.0108 & -0.0103 & -0.0098 & -0.0094 \\
\hline
\end{tabular}


Cuadro A.6: Indicadores macroeconómicos, Impuestos (9-16 años)

\begin{tabular}{|c|c|c|c|c|c|c|c|c|}
\hline Consumo & & & & & & & & \\
\hline Elasticidad & 4.1 & 4.5 & 4.9 & 5.3 & 5.7 & 6.1 & 6.5 & 6.9 \\
\hline Año & 9 & 10 & 11 & 12 & 13 & 14 & 15 & 16 \\
\hline Imp. $20 \%$ & -0.018 & -0.018 & -0.018 & -0.018 & -0.018 & -0.018 & -0.018 & -0.018 \\
\hline Imp. $40 \%$ & -0.024 & -0.024 & -0.024 & -0.024 & -0.024 & -0.024 & -0.024 & -0.024 \\
\hline Imp. $60 \%$ & -0.026 & -0.026 & -0.026 & -0.026 & -0.026 & -0.026 & -0.026 & -0.026 \\
\hline \multicolumn{9}{|l|}{ Inversión } \\
\hline Imp. $20 \%$ & -0.0033 & -0.0029 & -0.0026 & -0.0024 & -0.0023 & -0.0021 & -0.0020 & -0.0019 \\
\hline Imp. $40 \%$ & 0.0000 & 0.0003 & 0.0004 & 0.0003 & 0.0000 & -0.0001 & -0.0003 & -0.0004 \\
\hline Imp. $60 \%$ & 0.0005 & 0.0003 & 0.0001 & -0.0005 & -0.0010 & -0.0013 & -0.0016 & -0.0018 \\
\hline \multicolumn{9}{|l|}{ PIB } \\
\hline Imp. $20 \%$ & -0.0095 & -0.0092 & -0.0089 & -0.0086 & -0.0083 & -0.0081 & -0.0078 & -0.0076 \\
\hline Imp. $40 \%$ & -0.0101 & -0.0097 & -0.0093 & -0.0090 & -0.0087 & -0.0085 & -0.0083 & -0.0081 \\
\hline Imp. $60 \%$ & -0.0090 & -0.0087 & -0.0084 & -0.0082 & -0.0080 & -0.0079 & -0.0078 & -0.0077 \\
\hline
\end{tabular}

Cuadro A.7: Indicadores macroeconómicos, Impuestos (17-25 años)

\begin{tabular}{|c|c|c|c|c|c|c|c|c|c|}
\hline Consumo & & & & & & & & & \\
\hline Elasticidad & 7.3 & 7.7 & 8.1 & 8.5 & 8.9 & 9.3 & 9.7 & 9.9 & 10 \\
\hline Año & 17 & 18 & 19 & 20 & 21 & 22 & 23 & 24 & 25 \\
\hline Imp. $20 \%$ & -0.018 & -0.018 & -0.018 & -0.018 & -0.018 & -0.018 & -0.018 & -0.018 & -0.018 \\
\hline Imp. $40 \%$ & -0.024 & -0.024 & -0.024 & -0.024 & -0.024 & -0.024 & -0.024 & -0.024 & -0.024 \\
\hline Imp. $60 \%$ & -0.026 & -0.026 & -0.026 & -0.026 & -0.026 & -0.026 & -0.026 & -0.026 & -0.026 \\
\hline \multicolumn{10}{|l|}{ Inversión } \\
\hline Imp. 20\% & -0.0019 & -0.0019 & -0.0017 & -0.0017 & -0.0019 & -0.0020 & -0.0023 & -0.0021 & -0.0021 \\
\hline Imp. $40 \%$ & -0.0006 & -0.0009 & -0.0008 & -0.0008 & -0.0012 & -0.0014 & -0.0016 & -0.0016 & -0.0016 \\
\hline Imp. $60 \%$ & -0.0020 & -0.0022 & -0.0022 & -0.0023 & -0.0024 & -0.0024 & -0.0024 & -0.0025 & -0.0026 \\
\hline \multicolumn{10}{|l|}{ PIB } \\
\hline Imp. $20 \%$ & -0.0074 & -0.0073 & -0.0071 & -0.0070 & -0.0068 & -0.0067 & -0.0066 & -0.0066 & \\
\hline Imp. $40 \%$ & -0.0079 & -0.0078 & -0.0078 & -0.0077 & -0.0076 & -0.0075 & -0.0075 & -0.0075 & \\
\hline Imp. $60 \%$ & -0.0077 & -0.0077 & -0.0077 & -0.0077 & -0.0076 & -0.0077 & -0.0077 & -0.0078 & \\
\hline
\end{tabular}


Cuadro A.8: Indicadores macroeconómicos, Subs. (0-8 años)

Indicadores macroeconómicos

Subsidios y elasticidad variable

\begin{tabular}{|c|c|c|c|c|c|c|c|c|c|}
\hline Consumo & & & & & & & & & \\
\hline Elasticidad & 0.5 & 0.9 & 1.3 & 1.7 & 2.1 & 2.5 & 2.9 & 3.3 & 3.7 \\
\hline Año & 0 & 1 & 2 & 3 & 4 & 5 & 6 & 7 & 8 \\
\hline Subs. $20 \%$ & 0.0056 & 0.0056 & 0.0056 & 0.0056 & 0.0056 & 0.0056 & 0.0056 & 0.0056 & 0.0056 \\
\hline Subs. $40 \%$ & 0.0225 & 0.0225 & 0.0225 & 0.0225 & 0.0225 & 0.0225 & 0.0225 & 0.0225 & 0.0225 \\
\hline Subs. $60 \%$ & 0.0694 & 0.0694 & 0.0694 & 0.0694 & 0.0694 & 0.0694 & 0.0694 & 0.0694 & 0.0694 \\
\hline \multicolumn{10}{|l|}{ Inversión } \\
\hline Subs. $20 \%$ & 0.0143 & 0.0145 & 0.0148 & 0.0151 & 0.0153 & 0.0156 & 0.0159 & 0.0161 & 0.0163 \\
\hline Subs. $40 \%$ & 0.0559 & 0.0571 & 0.0583 & 0.0594 & 0.0603 & 0.0612 & 0.0618 & 0.0624 & 0.0627 \\
\hline Subs. $60 \%$ & 0.1642 & 0.1676 & 0.1702 & 0.1718 & 0.1723 & 0.1716 & 0.1701 & 0.1678 & 0.1653 \\
\hline \multicolumn{10}{|l|}{ PIB } \\
\hline Subs. $20 \%$ & 0.0077 & 0.0078 & 0.0079 & 0.0079 & 0.0080 & 0.0081 & 0.0082 & 0.0083 & 0.0084 \\
\hline Subs. $40 \%$ & 0.0307 & 0.0310 & 0.0313 & 0.0316 & 0.0319 & 0.0322 & 0.0325 & 0.0328 & 0.0330 \\
\hline Subs. $60 \%$ & 0.0929 & 0.0936 & 0.0942 & 0.0948 & 0.0952 & 0.0955 & 0.0958 & 0.0960 & 0.0962 \\
\hline
\end{tabular}

Cuadro A.9: Indicadores macroeconómicos, Subs. (9-16 años)

\begin{tabular}{|c|c|c|c|c|c|c|c|c|}
\hline Consumo & & & & & & & & \\
\hline Elasticidad & 4.1 & 4.5 & 4.9 & 5.3 & 5.7 & 6.1 & 6.5 & 6.9 \\
\hline Año & 9 & 10 & 11 & 12 & 13 & 14 & 15 & 16 \\
\hline Subs. $20 \%$ & 0.0056 & 0.0056 & 0.0056 & 0.0056 & 0.0056 & 0.0056 & 0.0056 & 0.0056 \\
\hline Subs. $40 \%$ & 0.0225 & 0.0225 & 0.0225 & 0.0225 & 0.0225 & 0.0225 & 0.0225 & 0.0225 \\
\hline Subs. $60 \%$ & 0.0694 & 0.0694 & 0.0694 & 0.0694 & 0.0694 & 0.0694 & 0.0694 & 0.0694 \\
\hline \multicolumn{9}{|l|}{ Inversión } \\
\hline Subs. $20 \%$ & 0.0165 & 0.0168 & 0.0169 & 0.0170 & 0.0169 & 0.0170 & 0.0170 & 0.0170 \\
\hline Subs. $40 \%$ & 0.0629 & 0.0630 & 0.0630 & 0.0622 & 0.0614 & 0.0610 & 0.0606 & 0.0602 \\
\hline Subs. $60 \%$ & 0.1629 & 0.1607 & 0.1589 & 0.1563 & 0.1543 & 0.1534 & 0.1527 & 0.1520 \\
\hline \multicolumn{9}{|l|}{ PIB } \\
\hline Subs. $20 \%$ & 0.0085 & 0.0086 & 0.0087 & 0.0088 & 0.0089 & 0.0090 & 0.0091 & 0.0091 \\
\hline Subs. $40 \%$ & 0.0333 & 0.0336 & 0.0339 & 0.0341 & 0.0343 & 0.0346 & 0.0348 & 0.0351 \\
\hline Subs. $60 \%$ & 0.0965 & 0.0967 & 0.0971 & 0.0975 & 0.0979 & 0.0983 & 0.0987 & 0.0990 \\
\hline
\end{tabular}


Cuadro A.10: Indicadores macroeconómicos, Subs. (17-25 años)

\begin{tabular}{|c|c|c|c|c|c|c|c|c|c|}
\hline Consumo & & & & & & & & & \\
\hline Elasticidad & 7.3 & 7.7 & 8.1 & 8.5 & 8.9 & 9.3 & 9.7 & 9.9 & 10 \\
\hline Año & 17 & 18 & 19 & 20 & 21 & 22 & 23 & 24 & 25 \\
\hline Subs. $20 \%$ & 0.0056 & 0.0056 & 0.0056 & 0.0056 & 0.0056 & 0.0056 & 0.0056 & 0.0056 & 0.0056 \\
\hline Subs. $40 \%$ & 0.0225 & 0.0225 & 0.0225 & 0.0225 & 0.0225 & 0.0225 & 0.0225 & 0.0225 & 0.0225 \\
\hline Subs. $60 \%$ & 0.0694 & 0.0694 & 0.0694 & 0.0694 & 0.0694 & 0.0694 & 0.0694 & 0.0694 & 0.0694 \\
\hline \multicolumn{10}{|l|}{ Inversión } \\
\hline Subs. $20 \%$ & 0.0170 & 0.0169 & 0.0171 & 0.0171 & 0.0168 & 0.0165 & 0.0163 & 0.0164 & 0.0164 \\
\hline Subs. $40 \%$ & 0.0598 & 0.0593 & 0.0596 & 0.0595 & 0.0589 & 0.0584 & 0.0580 & 0.0581 & 0.0581 \\
\hline Subs. $60 \%$ & 0.1515 & 0.1510 & 0.1511 & 0.1510 & 0.1503 & 0.1498 & 0.1493 & 0.1492 & 0.1491 \\
\hline \multicolumn{10}{|l|}{ PIB } \\
\hline Subs. $20 \%$ & 0.0092 & 0.0093 & 0.0094 & 0.0094 & 0.0095 & 0.0095 & 0.0096 & 0.0096 & \\
\hline Subs. $40 \%$ & 0.0353 & 0.0355 & 0.0357 & 0.0359 & 0.0361 & 0.0363 & 0.0365 & 0.0365 & \\
\hline Subs. $60 \%$ & 0.0994 & 0.0997 & 0.0998 & 0.1000 & 0.1003 & 0.1005 & 0.1006 & & \\
\hline
\end{tabular}


Cuadro A.11: Balanza comercial Imp. vs Subs. (0-14 años, \%)

\section{Balanza comercial}

\begin{tabular}{|c|c|c|c|c|c|c|c|c|c|c|}
\hline \multirow{4}{*}{$\begin{array}{c}\text { Elas. } \\
\text { Año } \\
\text { Referencia }\end{array}$} & Imps. & Subs. & Imps. & Subs. & Imps. & Subs. & Imps. & Subs. & Imps. & Subs. \\
\hline & \multicolumn{2}{|c|}{0.5} & \multicolumn{2}{|c|}{0.9} & \multicolumn{2}{|c|}{1.3} & \multicolumn{2}{|c|}{1.7} & \multicolumn{2}{|c|}{2.1} \\
\hline & \multicolumn{2}{|c|}{$\mathbf{0}$} & \multicolumn{2}{|c|}{1} & \multicolumn{2}{|c|}{2} & \multicolumn{2}{|c|}{3} & \multicolumn{2}{|c|}{4} \\
\hline & 0 & 0 & 0.0200 & 0.0200 & 0.0404 & 0.0404 & 0.0612 & 0.0612 & 0.0824 & 0.0824 \\
\hline $20 \%$ & -0.1382 & 0.0079 & -0.1496 & 0.0071 & -0.1608 & 0.0062 & -0.1719 & 0.0052 & -0.1828 & 0.0039 \\
\hline $40 \%$ & -0.2390 & 0.0293 & -0.2557 & 0.0294 & -0.2724 & 0.0285 & -0.2890 & 0.0266 & -0.3056 & 0.0233 \\
\hline $60 \%$ & -0.3196 & 0.0853 & -0.3390 & 0.0889 & -0.3585 & 0.0878 & -0.3782 & 0.0808 & -0.3977 & 0.0676 \\
\hline
\end{tabular}

\begin{tabular}{|c|c|c|c|c|c|c|c|c|c|c|}
\hline & Imps. & Subs. & Imps. & Subs. & Imps. & Subs. & Imps. & Subs. & Imps. & Subs. \\
\hline Elas. & \multicolumn{2}{|c|}{2.5} & \multicolumn{2}{|c|}{2.9} & \multicolumn{2}{|c|}{3.3} & \multicolumn{2}{|c|}{3.7} & \multicolumn{2}{|c|}{4.1} \\
\hline Año & \multicolumn{2}{|c|}{5} & \multicolumn{2}{|c|}{6} & \multicolumn{2}{|c|}{7} & \multicolumn{2}{|c|}{8} & \multicolumn{2}{|c|}{9} \\
\hline Referencia & 0.1041 & 0.1041 & 0.1262 & 0.1262 & 0.1487 & 0.1487 & 0.1717 & 0.1717 & 0.1951 & 0.1951 \\
\hline $20 \%$ & -0.1936 & 0.0026 & -0.2043 & 0.0010 & -0.2148 & -0.0007 & -0.2251 & -0.0026 & -0.2352 & -0.0047 \\
\hline $40 \%$ & -0.3219 & 0.0188 & -0.3379 & 0.0129 & -0.3536 & 0.0057 & -0.3688 & -0.0030 & -0.3835 & -0.0129 \\
\hline $60 \%$ & -0.4169 & 0.0479 & -0.4356 & 0.0225 & -0.4534 & -0.0073 & -0.4703 & -0.0402 & -0.4860 & -0.0744 \\
\hline
\end{tabular}

\begin{tabular}{|c|c|c|c|c|c|c|c|c|c|c|}
\hline \multirow{4}{*}{$\begin{array}{c}\text { Elas. } \\
\text { Año } \\
\text { Referencia }\end{array}$} & Imps. & Subs. & Imps. & Subs. & Imps. & Subs. & Imps. & Subs. & Imps. & Subs. \\
\hline & \multirow{2}{*}{\multicolumn{2}{|c|}{$\begin{array}{c}4.5 \\
10\end{array}$}} & \multirow{2}{*}{\multicolumn{2}{|c|}{$\begin{array}{r}4.9 \\
11\end{array}$}} & \multirow{2}{*}{\multicolumn{2}{|c|}{$\begin{array}{c}5.3 \\
12\end{array}$}} & \multirow{2}{*}{\multicolumn{2}{|c|}{$\begin{array}{l}5.7 \\
13\end{array}$}} & \multicolumn{2}{|c|}{6.1} \\
\hline & & & & & & & & & & 4 \\
\hline & 0.2190 & 0.2190 & 0.2434 & 0.2434 & 0.2682 & 0.2682 & 0.2936 & 0.2936 & 0.3195 & 0.3195 \\
\hline $20 \%$ & -0.2452 & -0.0070 & -0.2550 & -0.0095 & -0.2676 & -0.0133 & -0.2799 & -0.0175 & -0.2891 & -0.0207 \\
\hline $40 \%$ & -0.3976 & -0.0239 & -0.4110 & -0.0360 & -0.4277 & -0.0537 & -0.4432 & -0.0724 & -0.4542 & -0.0869 \\
\hline $60 \%$ & -0.5005 & -0.1088 & -0.5136 & -0.1425 & -0.5286 & -0.1853 & -0.5414 & -0.2253 & -0.5495 & -0.2535 \\
\hline
\end{tabular}


Cuadro A.12: Balanza comercial Imp. vs Subs. (15-25 años, \%)

\begin{tabular}{|c|c|c|c|c|c|c|c|c|c|c|}
\hline & Imps. & Subs. & Imps. & Subs. & Imps. & Subs. & Imps. & Subs. & Imps. & Subs. \\
\hline Elas. & \multicolumn{2}{|c|}{6.5} & \multicolumn{2}{|c|}{6.9} & \multicolumn{2}{|c|}{7.3} & \multicolumn{2}{|c|}{7.7} & \multicolumn{2}{|c|}{8.1} \\
\hline Año & \multicolumn{2}{|c|}{15} & \multicolumn{2}{|c|}{16} & \multicolumn{2}{|c|}{17} & \multicolumn{2}{|c|}{18} & \multicolumn{2}{|c|}{19} \\
\hline Referencia & 0.3459 & 0.3459 & 0.3728 & 0.3728 & 0.4002 & 0.4002 & 0.4282 & 0.4282 & 0.4568 & 0.4568 \\
\hline $20 \%$ & -0.2981 & -0.0242 & -0.3069 & -0.0278 & -0.3155 & -0.0317 & -0.3240 & -0.0358 & -0.3271 & -0.0372 \\
\hline $40 \%$ & -0.4644 & -0.1017 & -0.4737 & -0.1167 & -0.4824 & -0.1316 & -0.4902 & -0.1465 & -0.4926 & -0.1521 \\
\hline $60 \%$ & -0.5564 & -0.2799 & -0.5620 & -0.3044 & -0.5665 & -0.3272 & -0.5699 & -0.3483 & -0.5703 & -0.3547 \\
\hline
\end{tabular}

\begin{tabular}{|c|c|c|c|c|c|c|c|c|c|c|c|}
\hline Elas. & $\begin{array}{r}\text { Imps. } \\
8 .\end{array}$ & $\begin{array}{l}\text { Subs. } \\
5\end{array}$ & $\begin{array}{r}\text { Imps. } \\
8 .\end{array}$ & $\begin{array}{l}\text { Subs. } \\
.9\end{array}$ & $\begin{array}{r}\text { Imps. } \\
9 .\end{array}$ & Subs. & $\begin{array}{r}\text { Imps. } \\
9 .\end{array}$ & $\begin{array}{l}\text { Subs. } \\
.7\end{array}$ & $\begin{array}{r}\text { Imps. } \\
9\end{array}$ & Subs. & $\begin{array}{c}\text { Imps. Subs. } \\
10\end{array}$ \\
\hline Ã̃o & 20 & & 2 & 1 & 2 & 2 & 2 & 3 & & 4 & 25 \\
\hline Referencia & 0.486 & 0.486 & 0.516 & 0.516 & 0.546 & 0.546 & 0.577 & 0.577 & 0.608 & 0.608 & 0.641 \\
\hline $20 \%$ & -0.333 & -0.040 & -0.343 & -0.046 & -0.351 & -0.051 & -0.358 & -0.056 & -0.359 & -0.056 & $-0.362-0.058$ \\
\hline $40 \%$ & -0.497 & -0.162 & -0.505 & -0.181 & -0.510 & -0.195 & -0.515 & -0.209 & -0.515 & -0.210 & $-0.516-0.216$ \\
\hline $60 \%$ & -0.571 & -0.367 & -0.574 & -0.390 & -0.574 & -0.406 & -0.574 & -0.420 & -0.573 & -0.419 & $-0.572-0.422$ \\
\hline
\end{tabular}


MODELOS DE EOUILIBRTO

GENERAI Y IA INCORPORACION

DE ENERGLAS RENOVABLES

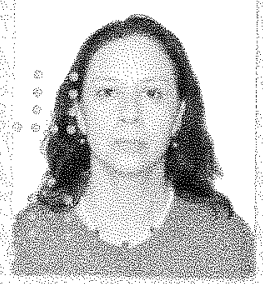

Clua drañáa Puldo

ELSA PATRICIA OMAÑA PULLDO ALUMNA

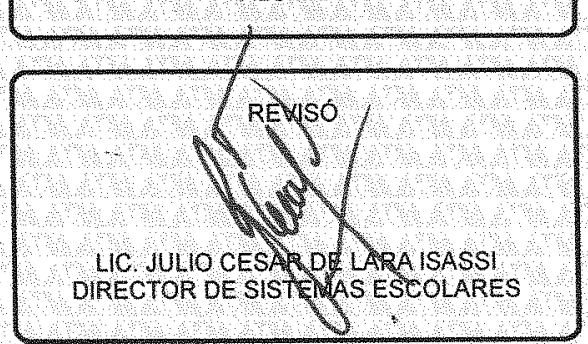

DIRECTOR DE LA DIVISION DE CB

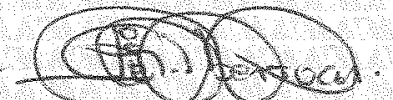

DR, JOSE GILBERTO CORDOBA HERRERA

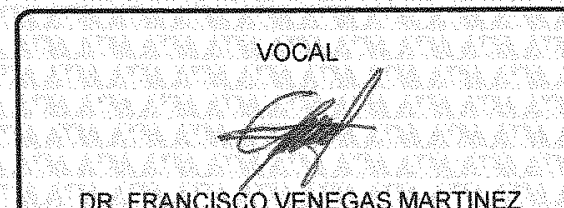

DR. FRANCISCO VENEGAS MARTINEZ
En Ia Ciudad de México, se presentaron a las 12:00 horas del dia 10 del mes de noviembre del año 2017 en la unidad Iztapalapa de la universidad Autónoma Metropolitana, los suscritos niembros del jurado.

DR. MANUET ORDORICA MELIADO

DR. JOAOUTN DEIGADO FERNANDEZ

DR. FRANCISCO VENEGAS MARTINEZ

DR. PEDRO LARA VELAZOUEZ

DR. MIGUET ANGEL GUTTERREZ ANDRADE

Bajo la presidencia del primero y con caracter de Secretario el último, se reunieron a la presentación de la Disertación Pública cuya denominación aparece al margen, para la obtención del grado de:

DOCTORA EN CIENCIAS (CIENCIAS Y TECNOLOGIAS DE LA INEORMAC ION)

DE: ELSA PATRICIA OMANIA PULIDO

y de acuerdo con el artículo 78 fracción IV del Reglamento de Estudios Superlores de la Universtad Futonoma Metropolitana, los miembros del Jurado resolvieron:

\section{Aprobar}

Acto continuo, el presidente del jurado comunicó a la interesada el resultado de la evaluación y, en caso aprobatorio, le fue tomada la protesta.
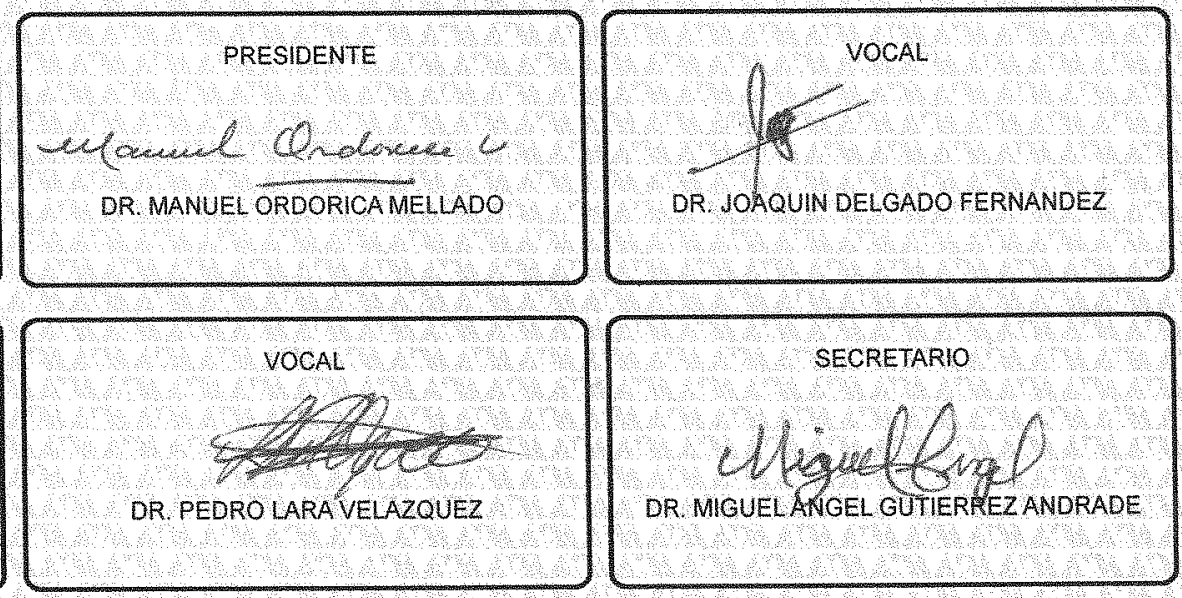